DEPARTMENT OF THE INTERIOR

U.S. GEOLOGICAL SURVEY

\title{
Analytical data and sample locality map of stream-sediment and heavy-mineral-concentrate samples collected from the Horn Mountains area, sleetmute quadrangle, southwest Alaska
}

\author{
By \\ P.M. Theodorakos, J.C. Borden, J.H. Bullock, Jr., \\ J.E. Gray, and P.L. Hageman \\ Open-File Report 92-708-A Paper version \\ 92-708-B Diskette version
}

This report is preliminary and has not been reviewed for conformity with U.S. Geological Survey editorial standards and stratigraphic nomenclature. Any use of trade names is for descriptive purposes only and does not imply endorsement by the USGS.

U.S. Geological Survey, DFC, Box 25046, MS 973, Denver, Co, 80225 


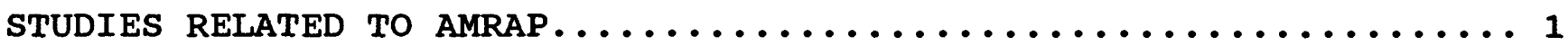

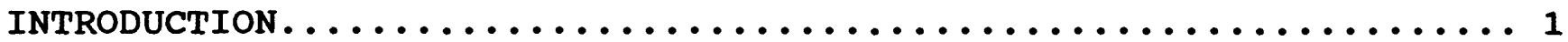

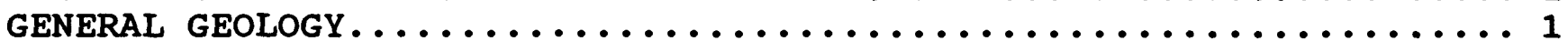

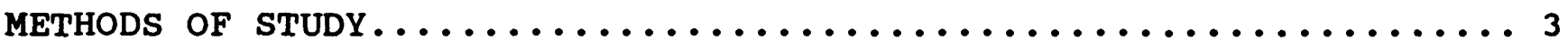

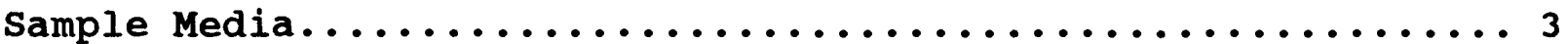

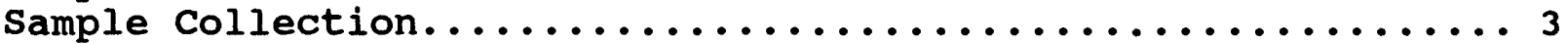

stream-sediment samples..........................

Heavy-mineral-concentrate samples...................5

Sample Preparation...................................

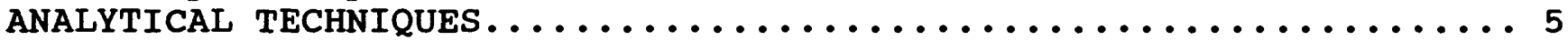

Semiquantitative Emission spectrography.................

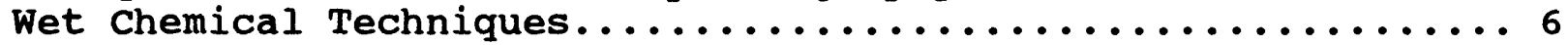

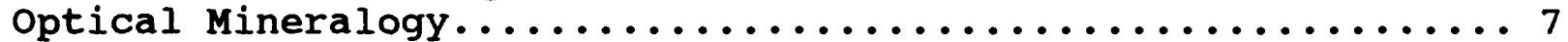

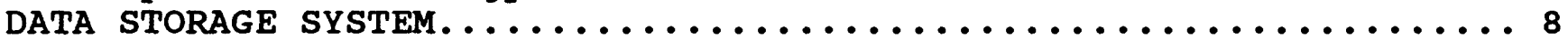

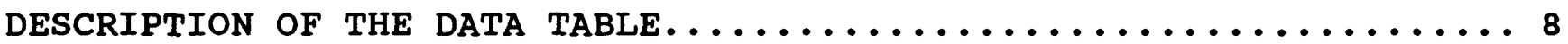

ACKNOWLEDGMENTS ......................................

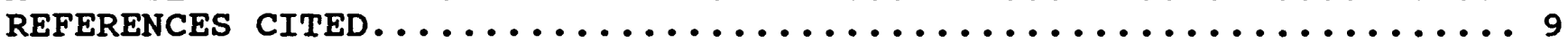

\section{ILLUSTRATIONS}

Figure 1. Location of the Horn Mountains study area, sleetmute quadrangle.........................

Figure 2. Localities of samples collected from the Horn Mountains study area in the sleetmute quadrangle ..............4

\section{TABLES}

Table 1. Limits of determination for spectrographic analysis of stream-sediment and heavy-mineral-concentrate samples... 11

Table 2. Other analytical methods used and limits

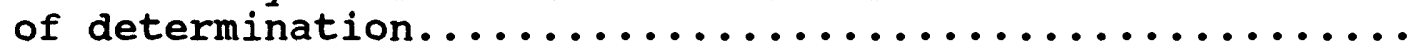

Table 3. Analytical data for stream-sediment samples collected from the Horn Mountains area, sleetmute quadrangle, Alaska.

Table 4. Analytical data for heavy-mineral-concentrate samples collected from the Horn Mountains area, sleetmute quadrangle, Alaska..........................

Table 5. Mineralogical data for heavy-mineral-concentrate samples from the Horn Mountains area, sleetmute quadrangle, Alaska................................... 


\section{STUDIES RELATED TO AMRAP}

The U.S. Geological Survey is required by the Alaska National Interests Lands Conservation Act (Public Law 96-487, 1980) to survey certain federal lands to determine their mineral potential. Results from the Alaska Mineral Resource Assessment Program (AMRAP) must be made available to the public and submitted to the President and congress. This report is one of a series of publications that presents geochemical data collected during the mineral assessment study of the sleetmute quadrangle, Alaska (fig. 1). Geochemical data for stream-sediment samples, and geochemical and mineralogical data for heavy-mineral-concentrate samples collected from the Horn Mountains and surrounding areas are presented here. The data in this report are also available on computer diskette in Theodorakos and others (1992). An interpretation of the data appears in Gray and others (in press).

\section{INTRODUCTION}

In the summer of 1992, a reconnaissance geochemical survey was conducted in the northwestern part of the sleetmute quadrangle as part of the Alaskan Mineral Resource Assessment Program (AMRAP). The study area covers approximately $1500 \mathrm{~km}^{2}\left(579 \mathrm{mi}^{2}\right)$ and includes the Horn Mountains and surrounding areas (fig. 2). Reconnaissance drainage basin geochemical surveys are a rapid and efficient means of locating upstream areas with possible mineral deposits. The objective of this study was to use geochemical data from stream-sediment samples, and geochemical and mineralogical data from heavy-mineral-concentrate samples, to identify areas favorable for the presence of mineral deposits.

The most rugged topography of the study area is in the Horn Mountains where the maximum elevation is approximately $1071 \mathrm{~m} \mathrm{(3515}$ ft). However, much of the terrain lying outside of the Horn Mountains is dominated by low rolling hills with broad, sediment-filled lowlands. Some of the study area is swampy, especially along part of the Kolmakof River where the minimum elevation is approximately $60 \mathrm{~m}$ $(200 \mathrm{ft})$. The region is covered with vegetation that ranges from northern latitude forests to subarctic tundra. No roads are found in the area and access is limited to travel by air or foot. Boat access is also possible on some of the larger rivers and creeks.

\section{GENERAL GEOLOGY}

The Horn Mountains are made-up of one of several Late cretaceous to early Tertiary volcanoplutonic complexes found in southwestern Alaska. Volcanic rocks of these complexes generally overlie or are in fault contact with plutons that intrude and partially assimilate the volcanic rocks (Bundtzen and Gilbert, 1983). The plutonic rocks in the Horn Mountains are part of a stock of mostly quartz monzonite (Cady and others, 1955). This stock intrudes Late cretaceous and early Tertiary mafic to felsic tuffs and lavas of the Iditarod Volcanics (Miller and Bundtzen, 1988), and sedimentary rocks of the Cretaceous Kuskokwim Group (Cady and others, 1955). Contact metamorphic aureoles are found adjacent to the plutons where sedimentary and volcanic rocks are converted to hornfels.

Late Cretaceous and early Tertiary intermediate to mafic dikes and granite porphyry intrusions are found in the vicinity of the Horn 


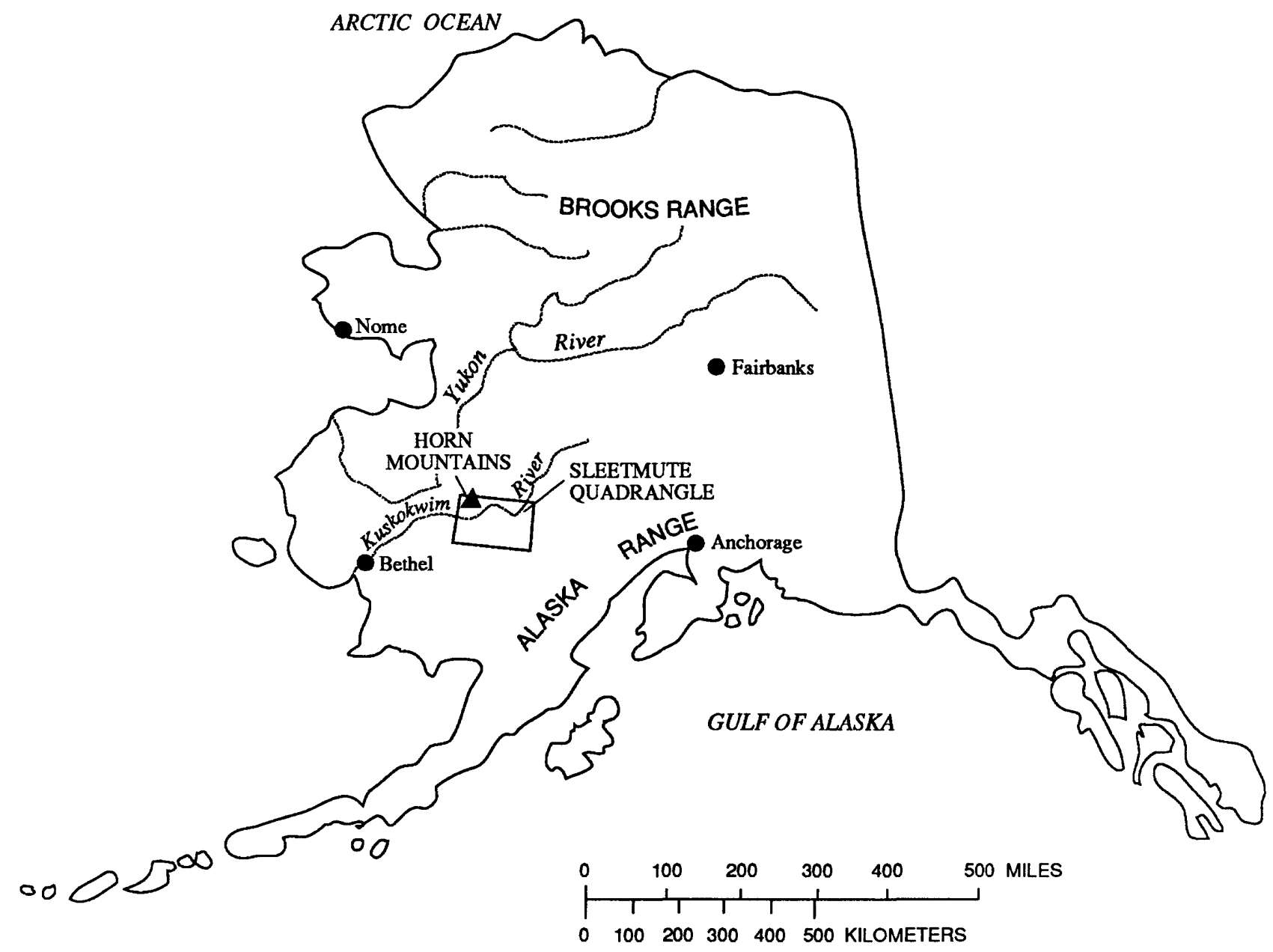

Figure 1. Location of the Horn Mountains area, sleetmute quadrangle, Alaska. 
Mountains. These intrusions cut all rock types in the study area, but most commonly intrude sedimentary rocks of the kuskokwim Group. The largest exposure of granite porphyry is at Juninggulra Mountain approximately $20 \mathrm{~km}$ north of the Horn Mountains. The intermediate to mafic dikes are generally small and local, typically less than 1 meter wide, discontinuous along strike, and difficult to trace for more than 10 meters (Cady and others, 1955; Miller and Bundtzen, 1993).

Rocks of the Cretaceous Kuskokwim Group surround the Horn

Mountains. The Kuskokwim Group is a sequence of flysch representing turbidite fan, foreslope, shallow-marine, and shelf facies deposited into a Late Cretaceous basin (Bundtzen and Gilbert, 1983; Miller and Bundtzen, 1992). Rocks of the Kuskokwim Group were first described and named by Cady and others (1955), who suggested graywacke and lesser siltstone compose almost all of the sequence, graywacke being approximately twice as abundant as siltstone. Minor conglomerate and interbedded volcanic tuffs and flows are also found within the Kuskokwim Group (Cady and others, 1955; Bundtzen and Laird, 1982 ; Miller and Bundtzen, 1993).

\section{Sample Media}

\section{METHODS OF STUDY}

In this reconnaissance geochemical study, stream-sediment and heavy-mineral-concentrate samples were collected to provide information about the rocks eroded from the drainage basin upstream from each sample site. Analyses of stream-sediment samples are representative of the chemistry of rocks contained within the drainage basins. Heavy-mineral-concentrate samples provide information about the more dense minerals in rocks eroded from the drainage basins. The heavy-mineral-concentrate collection procedure selectively concentrates the dense minerals, many of which may be related to ore deposits, permitting chemical determination of some elements that are not easily detected in stream-sediment samples. The mineralogical content of the heavy-mineral-concentrate samples was also determined to provide additional information that may help to delineate mineral deposits. All of this information is useful for identifying ground favorable for mineral deposits.

\section{sample collection}

Stream-sediment samples were collected from 138 sites from firstand second-order streams (fig. 2). Heavy-mineral-concentrates were collected from 137 sites. At sample site (HM0127), a heavy-mineralconcentrate sample was not collected because the stream drainage was swampy and lacked sufficient sand-sized material necessary for concentrate collection. Sampling density was approximately one site per $11 \mathrm{~km}^{2}\left(4.2 \mathrm{mi}^{2}\right)$. The area of the drainage basin sampled ranged from approximately $2.6 \mathrm{~km}^{2}\left(1 \mathrm{mi}^{2}\right)$ to $15 \mathrm{~km}^{2}\left(5.8 \mathrm{mi}^{2}\right)$.

\section{stream-sediment samples}

Stream-sediment material collected consisted of alluvium in the active stream channel. When possible, samples were composited by collecting sediment from several localities in the active channel. However, some sites were swampy, with deep channels where it was not possible to identify the active channel bottom. In these instances, 


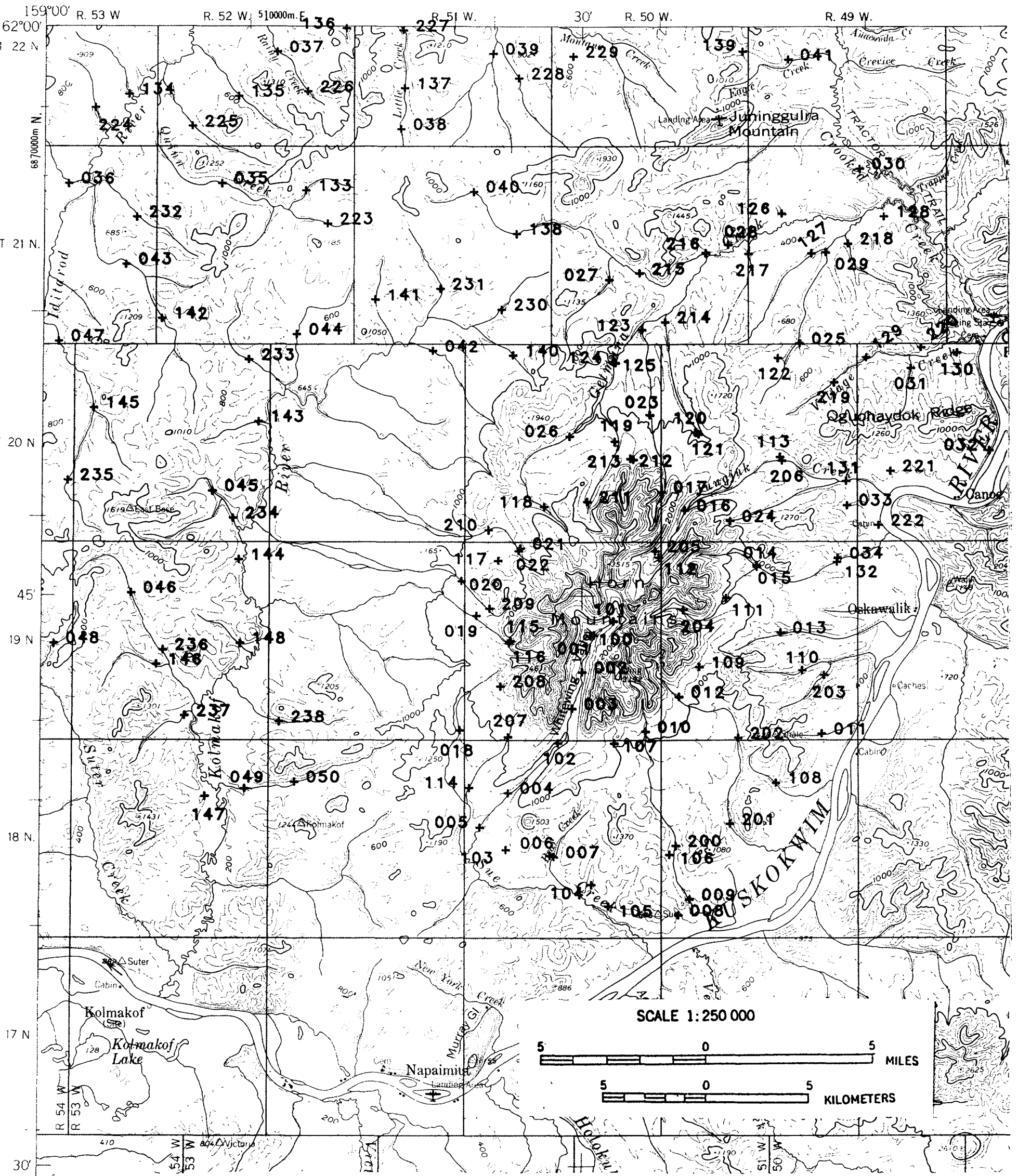

Figure 2. Localities of samples collected from the Horn Mountains study area in the Sleetmute quadrangle. 
any available sediment was collected from the channel bottom using specialized shovels with extendable handles capable of reaching into these deep streams. The stream sediment was then screened to minus-10 mesh and collected in a stainless steel gold pan. Approximately $2 \mathrm{~kg}$ of sediment was taken from the pan and saved as the stream-sediment sample.

Heavy-mineral-concentrate samples

Heavy-mineral-concentrates were collected from the same active alluvium as the stream-sediment samples. At each sample site, the gold pan was filled with stream sediment screened to minus-10 mesh and panned until most of the less dense minerals, organic materials, and clays were removed. This panned sample was saved as the heavymineral-concentrate sample. Streams sampled in areas covered by swampy lowlands often contain bed load material consisting primarily of clay with minor sand-sized material. In these instances, the gold pan was refilled several times to obtain adequate sand-sized material necessary for a heavy-mineral-concentrate sample.

\section{Sample Preparation}

In the laboratory, all 138 stream-sediment samples were dried below $50^{\circ} \mathrm{C}$, sieved to minus -80 mesh, pulverized, and chemically analyzed. The heavy-mineral-concentrate samples were sieved to minus35 mesh, and then separated using bromoform (specific gravity 2.85) to remove any remaining lighter minerals, primarily quartz and feldspar. The resultant heavy-mineral-concentrate samples were then separated into magnetic, paramagnetic, and nonmagnetic fractions using a modified Frantz Isodynamic Separator. The most magnetic material was removed at a setting of 0.25 ampere and contains mostly magnetite. The paramagnetic fraction was removed at 1.75 ampere and consists largely of ferromagnesian silicates and iron oxides. The nonmagnetic fraction of the heavy-mineral-concentrate samples contains sulfide minerals, gold, and some nonmagnetic oxides and silicates; this was the only fraction chemically analyzed. The nonmagnetic heavy-mineralconcentrate samples were split using a Jones-type multiple plate splitter; one split was hand ground and chemically analyzed and the other split was used for mineralogical analysis.

All of the 137 heavy-mineral-concentrate samples collected were examined microscopically for their mineralogical content. Following heavy-liquid and magnetic separation one heavy-mineral-concentrate sample (HM0029c) lacked sufficient material necessary for chemical analysis, resulting in a total of 136 concentrates being chemically analyzed.

\section{Semiquantitative Emission spectrography}

\section{ANALYTICAL TECHNIQUES}

The minus-80-mesh stream-sediment and minus-35-mesh nonmagneticheavy-mineral-concentrate samples were analyzed by a semiquantitative, direct-current arc emission spectrographic (SQS) technique adapted from Grimes and Marranzino (1968). Spectrographic results were determined by visually comparing spectra derived from the sample against spectra obtained from laboratory reference standards made from pure oxides and carbonates. Standard concentrations are geometrically spaced over any given order of magnitude as follows: $100,50,20,10$, 
etc. Samples whose concentrations were estimated to fall between those values were assigned values of $70,30,15$, etc. The precision of this analytical technique is approximately \pm one reporting interval at the 83 percent confidence level and \pm two reporting intervals at the 96 percent confidence level (Motooka and Grimes, 1976). Values determined for the major elements $\mathrm{Fe}, \mathrm{Mg}, \mathrm{Ca}, \mathrm{Na}, \mathrm{Ti}$, and $\mathrm{P}$ are given in weight percent; all other values are in parts per million (micrograms/gram). In addition, $P d$ and $P t$ were determined in the heavy-mineral-concentrate samples by SQS. The elements determined by sQS and their limits of determination are listed in table 1. Data for stream-sediment samples determined by the sQs technique are listed in table 3; sos data for the heavy-mineral-concentrate samples are listed in table 4 .

\section{wet Chemical Techniques}

Concentrations of $\mathrm{Ag}, \mathrm{As}, \mathrm{Au}, \mathrm{Bi}, \mathrm{Cd}, \mathrm{Cu}, \mathrm{Mo}, \mathrm{Pb}, \mathrm{Sb}$, and $\mathrm{Zn}$ were determined in the minus-80-mesh stream-sediment samples by inductively coupled plasma-atomic emission spectroscopy (ICP-AES) using the procedure developed by Motooka (1988). The sediments were decomposed with concentrated hydrochloric acid and hydrogen peroxide in a hotwater bath. The metals were extracted in diisobutyl ketone (DIBK) in the presence of ascorbic acid and potassium iodide. The DIBK phase was then aspirated directly into the plasma and element concentrations were determined simultaneously with a multichannel ICP instrument. Concentrations of $\mathrm{Au}, \mathrm{Te}$, and $\mathrm{Tl}$ in the minus-80-mesh streamsediment samples were determined by an atomic absorption spectrophotometry (AAS) technique adapted from Hubert and Chao (1985). The samples were digested using a series of hydrogen peroxide, hydrofluoric acid, aqua-regia, and hydrobromic acid-bromine solutions. Gold, Te and $T 1$ were separated and concentrated by extraction into methyl isobutyl ketone and determined by flame AAS. Concentrations for Au in the range of 0.002 to $0.050 \mathrm{ppm}$ were determined by graphite furnace AAS on samples that were shown to be less than $0.050 \mathrm{ppm}$ by the flame AAS technique. The graphite furnace AAs technique for Au was adapted from Meier (1980).

The minus-80-mesh stream-sediment samples were analyzed for se using continuous-flow hydride generation AAS (Sanzolone and Chao, 1987). In this method, the stream-sediment samples were digested using nitric, perchloric, and hydrofluoric acids; hydrochloric acid was added to form Se (IV), which is necessary for hydride generation. A mixture of hydrochloric acid, sodium borohydride, and sodium hydroxide was added to produce selenium hydride, which is then stripped and transported with inert gas to the atomizer of the atomic absorption spectrophotometer where se concentration was determined.

Mercury was measured in the minus-80-mesh stream-sediment samples using a modified version of the cold-vapor AAS technique (Kennedy and crock, 1987). The samples were decomposed with nitric acid and sodium dichromate. Mercury (II) was reduced to mercury gas with

hydroxylamine hydrochloride/sodium chloride and stannous chloride in a continuous flow system releasing the gas into a quartz cell of an atomic absorption spectrophotometer where concentration was determined.

Tungsten was determined in the minus-80-mesh stream-sediment samples by a visible spectrophotometric (VS) method by decomposing the samples with nitric, hydrofluoric, and hydrochloric acids (Welsch, 
1983). Stannous chloride and dithiol solution was added to reduce the $\mathrm{w}$, forming the blue tungsten-dithiol complex, which was then extracted into heptane. The color intensity of the tungsten-dithiol complex is proportional to the concentration of $\mathrm{W}$ in the sample. Tungsten concentrations were determined using a visible absorption spectrophotometer.

Concentrations of $F$ were determined in the minus-80-mesh streamsediment samples by selective-ion electrode (SIE) analysis using a method modified from Bodkin (1977). Samples were fused with lithium metaborate, and then dissolved in nitric acid. A complexing buffer was added, and $\mathrm{F}$ concentrations were determined using a F-selective ion electrode.

Concentrations of the platinum group elements (PGE) Pt, Pd, Rh, $\mathrm{Ru}$, and $\mathrm{Ir}$ were determined in the minus-80-mesh stream-sediment samples using an inductively coupled plasma-mass spectrometry (ICP-MS) technique (Meier and others, 1991). Digestion and separation of the PGE was made using a nickel sulfide fire assay procedure similar to that described by Jackson and others (1990). The nickel sulfide button is dissolved in hydrochloric acid, tellurium is added and reduced with stannous chloride to coprecipitate PGE sulfides, which are retained by filtration. The residue is dissolved in aqua regia and the solution is analyzed for PGE using an ICP-MS instrument.

The elements determined by the various wet chemical techniques and their limits of determination are listed in table 2 . Data determined for the stream-sediment samples by these methods are shown in table 3. Discrepancies in analyses for certain elements duplicated by different analytical methods, such as values determined for Au in stream-sediment samples, may be attributable to the particulate nature of minerals that contain Au, different sample aliquots used, and different extraction procedures. The AAS analysis of Au provides the most statistically representative results due to the larger sample aliquot analyzed. For example, a 10-gram sample aliquot is used for the AAS analysis, whereas a 10-milligram sample aliquot is used in the sQS technique.

\section{Optical Mineralogy}

Mineral identifications were made with a binocular microscope on each of the nonmagnetic, heavy-mineral-concentrate samples. The amount of a particular mineral in the concentrates was recorded in the following way: class $1,<1 \% ;$ class $2,1-5 \% ;$ class $3,>5-20 \%$; class 4 , $>20-50 \%$; and class $5,>50 \%$. If a particular mineral was not observed, an "0" was recorded. Mineralogy results are shown in table 5.

The following minerals were recognized in heavy-mineralconcentrate samples, abbreviations are listed here in parentheses and in the column headings in table 5: zircon (zir), apatite (apt), cassiterite (cass), rutile (rut), anatase (ant), brookite (brk), tourmaline (tour), pyroxene (pyx), sphene (spn), garnet (gar), fluorite (flu), pyrite (pyr), cinnabar (cinn), chalcopyrite (cpy), gold (gold), scheelite (schl), barite (bar), sphalerite (sphl), corundum (cor), muscovite (musc), andalusite (and), sillimanite (sil), monazite (monz), and chlorite (chl). 


\section{DATA STORAGE SYSTEM}

Upon completion of the analytical work, the results were entered into a computer-based file as part of the USGS Rock Analysis storage system (RASS) database. This database contains both descriptive geological information and analytical data. Any of this information may be retrieved and converted to a binary form (STATPAC) for computerized analysis or publication (VanTrump and Miesch, 1976).

The data in this report are also available on a 5.25 inch, 360-KB magnetic diskette in Theodorakos and others (1992). Access to this information requires an IBM compatible computer using MS DOS and a 5.25 inch drive capable of handling 360-KB diskettes. The diskette report contains the analytical results for the stream-sediment and heavy-mineral-concentrate samples in STATPAC file (.STP) format. An executable data conversion program STP2DAT.EXE (Grundy and Miesch, 1987) is also contained on the diskette that provides various format options into which the STATPAC file may be changed.

\section{DESCRIPTION OF DATA TABLE}

Table 3 contains the geochemical data for the stream-sediment samples collected during this study; tables 4 and 5 contain the geochemical and mineralogical data for the heavy-mineral-concentrate samples, respectively. Sample site locations are given in latitude and longitude in the tables, and these sample sites are plotted on figure 2. Sample site localities were abbreviated on figure 2 showing only a 3-digit number corresponding to sample numbers in the data tables.

The analytical method for each element shown in tables 3 and 4 is abbreviated as a suffix in the column headings. The designation "sQs" indicates semiquantitative emission spectrography, "ICP" indicates inductively coupled plasma-atomic emission spectroscopy and inductively coupled plasma-mass spectrometry, "AAS" indicates atomic absorption spectrophotometry, "Vs" indicates visible spectrophotometry, and "SIE" indicates selective-ion electrode analysis. The letter " $N$ " in the data table indicates that an element was looked for but not observed at the concentration shown, while an "<" indicates that an element was observed but present in concentrations below the lower limit of determination shown. A ">" was entered in the table after the upper limit of determination if an element was observed but was present in concentrations above this value. Lower and upper limits of determination for the ICP methods listed in tables 2 and 3 may be variable due to variable sample aliquot weight, dilution of an analytical aliquot, or instrumental interference correction.

\section{ACKNOWL EDGMENTS}

We would like thank Leon Bradley, Joe Curry, Jerry Motooka, Craig Motooka, Bruce Roushey, and John Sharkey for chemical analyses. Steve Sutley provided X-ray mineral identifications and Danny Abrams performed sample preparations. 


\section{REFERENCES CITED}

Bodkin, J.B., 1977, Determination of fluorine in silicates by use of an ion-selective electrode following fusion with lithium metaborate: The Analyst, v. 102, no. 1215, p. 409-413.

Bundtzen, T.K. and Gilbert, W.G., 1983, Outline of geology and mineral resources of upper Kuskokwim region, Alaska: Journal of the Alaska Geological Society, v. 3, p. 101-119.

Bundtzen, T.K., and Laird, G.M., 1982, Geologic map of the Iditarod D2 and eastern D-3 quadrangles, Alaska: Alaska Division of Geological and Geophysical Surveys, Geologic Report 72, 1 sheet, scale $1: 63,360$.

Cady, W.M., Wallace, R.E., Hoare, J.M., and Webber, E.J., 1955, The central Kuskokwim region, Alaska: U.S. Geological Survey

Professional Paper 268, $132 \mathrm{p}$.

Gray, J.E., Theodorakos, P.M., Bradley, L.A., and Bullock, J.H., Jr., in press, Favorable areas for metallic mineral resources in and near the Horn Mountains, sleetmute quadrangle, southwestern Alaska, in Dusel-Bacon, Cynthia, and Till, A.B., eds., Geologic studies in Alaska by the U.S. Geological Survey during 1992: U.S. Geological Survey Bulletin.

Grimes, D.J., and Marranzino, A.P., 1968, Direct-current arc and alternating-current spark emission spectrographic field methods for the semiquantitative analysis of geological materials: U.S. Geological survey Circular 591, 6 p.

Grundy, W.R., and Miesch, A.T., 1987, Brief descriptions of STATPAC and related statistical programs for the IBM personal computer: U.S. Geological Survey open-File Report 87-411-A, 34 p.

Hubert, A.E., and Chao, T.T., 1985, Determination of gold, indium, tellurium and thallium in the same sample digestion of geological materials by atomic-absorption spectroscopy and two-step solvent extraction: Talanta, v. 32, p. 383-387.

Jackson, S.E., Fryer, B.J., Gosse, W., Healey, D.C., Longerich, H.P., and Strong, D.F., 1990, Determination of the precious metals in geological materials by inductively coupled plasma-mass spectrometry (ICP-MS) with nickel sulphide fire-assay collection and tellurium coprecipitation, in Potts, P.J., Dupuy, C., and Bowles, J.F.W., eds., Microanalytical Methods in Mineralogy and Geochemistry: Chemical Geology, v. 83, p. 119-132.

Kennedy, K.R., and Crock, J.G., 1987, Determination of mercury in geological materials by continuous flow, cold-vapor, atomic-absorption spectrophotometry: Analytical Letters, v. 20, p. 899-908. 
Meier, A.L., 1980, Flameless atomic-absorption determination of gold in geological materials: Journal of Geochemical Exploration, $v$. 13 , p. 77-85.

Meier, A.L., Carlson, R.R., and Taggart, J.E., 1991, The determination of the platinum group elements in geologic materials by inductively coupled plasma mass spectrometry [abs.]: The Sixth Annual International Platinum Symposium, Perth, Australia, Abstracts with Programs.

Miller, M.L., and Bundtzen, T.K., 1988, Right-lateral offset solution for the Iditarod-Nixon Fork fault, western Alaska, in Galloway, J.P., and Hamilton, T.D., eds., Geologic studies in Alaska by the U.S. Geological Survey during 1987: U.S. Geological survey Circular 1016, p. 99-103.

Miller, M.L., and Bundtzen, T.K., 1992, Geologic history of the postaccretionary rocks, Iditarod quadrangle, west-central Alaska [abs.]: Geological Society of America Abstracts with Programs, v.24, no. 5 , p. 71 .

Miller, M.L., and Bundtzen, T.K., 1993, Geologic map of the Iditarod quadrangle, Alaska: U.S. Geological Survey Miscellaneous Field Studies Map MF-2219-A, scale 1:250,000.

Motooka, J.M., 1988, An exploration geochemical technique for the determination of preconcentrated organometallic halides by ICPAES: Applied Spectroscopy, v. 42, no. 7, p. 1293-1296.

Motooka, J. M., and Grimes, D. J., 1976, Analytical precision of onesixth order semiquantitative spectrographic analyses: U.S. Geological Survey Circular 738, 25 p.

Sanzolone, R.F., and Chao, T.T., 1987, Determination of selenium in thirty-two geochemical reference materials by continuous-flow hydride generation atomic absorption spectrophotometry: Geostandards Newsletter, v. 11, p. 81-85.

Theodorakos, P.M., Borden, J.C., Bullock, J.H., Jr., Gray, J.E., Hageman, P.L., 1992, Diskette version of analytical data for stream-sediment and heavy-mineral-concentrate samples collected from the Horn Mountains area, Sleetmute quadrangle, Alaska: U.S. Geological Survey open-File Report 92-708-B, 1-360 KB diskette.

VanTrump, George, Jr., and Miesch, A.T., 1976, The U.S. Geological Survey RASS-STATPAC system for management and statistical reduction of geochemical data: Computers and Geosciences, v. 3, p. $475-388$.

Welsch, E.P., 1983, A rapid geochemical spectrophotometric determination of tungsten with dithiol: Talanta, v. 30, p. 876-878. 
Table 1. Limits of determination for the spectrographic analysis of stream-sediment samples, based on a 10-mg sample.

[The spectrographic limits of determination for heavy-mineralconcentrate samples are based on a 5-mg sample weight, and are therefore two reporting intervals higher than the limits given for stream sediments]

Elements Lower determination limit Upper determination limit

Percent

\begin{tabular}{llr}
\hline Iron (Fe) & 0.05 & 20 \\
Magnesium (Mg) & 0.02 & 10 \\
Calcium (Ca) & 0.05 & 20 \\
Sodium (Na) & 0.2 & 5 \\
Titanium (Ti) & 0.002 & 1 \\
Phosphorous (P) & 0.2 & 10 \\
\hline
\end{tabular}

\begin{tabular}{|c|c|c|}
\hline silver (Aq) & 0.5 & 5.000 \\
\hline Arsenic (As) & 200 & 10,000 \\
\hline Gold (Au) & 10 & 500 \\
\hline Boron (B) & 10 & 2,000 \\
\hline Barium (Ba) & 20 & 5,000 \\
\hline Beryllium (Be) & 1 & 1,000 \\
\hline Bismuth (Bi) & 10 & 1,000 \\
\hline Cadmium (cd) & 20 & 500 \\
\hline Cobalt (Co) & 10 & 2,000 \\
\hline Chromium (Cr) & 10 & 5,000 \\
\hline Copper (Cu) & 5 & 20,000 \\
\hline Gallium (Ga) & 5 & 100 \\
\hline Germanium (Ge) & 10 & 100 \\
\hline Lanthanum (La) & 50 & 1,000 \\
\hline Manganese (Mn) & 10 & 5,000 \\
\hline Molybdenum (Mo) & 5 & 2,000 \\
\hline Niobium (Nb) & 20 & 2,000 \\
\hline Nickel (Ni) & 5 & 5,000 \\
\hline Lead (Pb) & 10 & 20,000 \\
\hline Antimony (Sb) & 100 & 10,000 \\
\hline Scandium (Sc) & 5 & 100 \\
\hline $\operatorname{Tin}(\mathrm{Sn})$ & 10 & 1,000 \\
\hline strontium (Sr) & 100 & 5,000 \\
\hline Thorium (Th) & 100 & 2,000 \\
\hline Vanadium (V) & 10 & 10,000 \\
\hline Tungsten (W) & 20 & 10,000 \\
\hline Yttrium (Y) & 10 & 2,000 \\
\hline Zinc $(\mathrm{Zn})$ & 200 & 10,000 \\
\hline Zirconium ( $\mathrm{Zr}$ ) & 10 & 1,000 \\
\hline Palladium (Pd) & 5 & 1,000 \\
\hline Platinum (Pt) & 20 & 1,000 \\
\hline
\end{tabular}

Determined in heavy-mineral-concentrate samples only. 
Table 2. Other analytical methods used and limits of determination*. [ICP-AES, inductively coupled plasma-atomic emission spectroscopy; AAS, atomic absorption spectrophotometry; VS, visible spectrophotometry; SIE, selective-ion electrode analysis; ICP-MS, inductively coupled plasma-mass spectrometry].

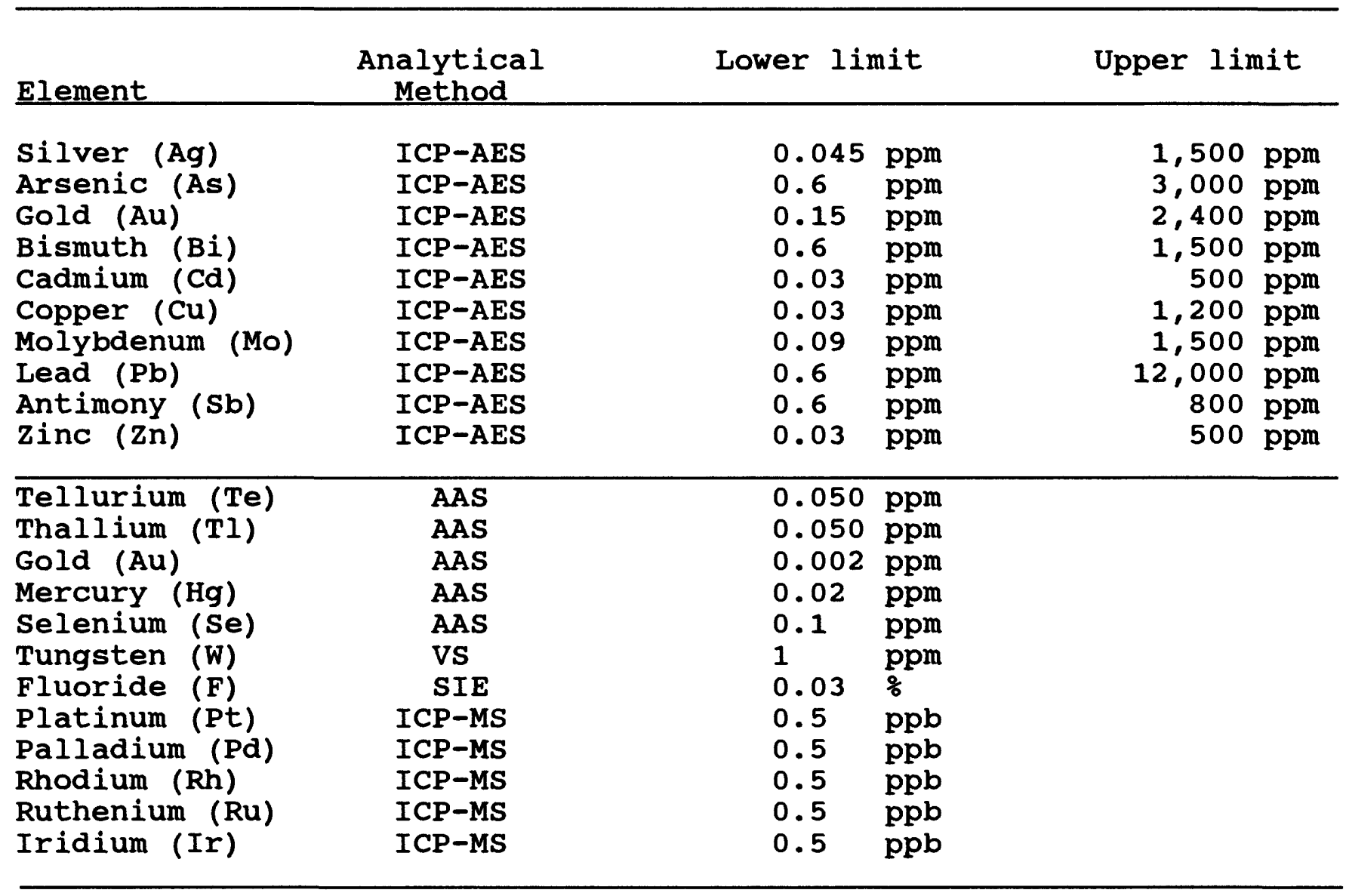

* NOTE: Limits of determination for the ICP-AES and ICP-MS methods listed in this table are nominal, and in table 3 may be variable. The variability in limits of determination for an element is due to variable sample aliquot weight, dilution of an analytical aliquot, or instrumental interference correction. 
Table 3. Analytical data for stream-sediment samples collected from the Horn Mountains area, Sleetmute quadrangle, Alaska. IICP, inductively coupled plasma spectroscopy; SQS, semiquant itative emission spectrography; MAS, atomic absorption

spectrophotometry; VS, visible spectrophotometry; SIE, selective-ion electrode analysis; N, not detected; <, detected but below the limit of determination shown; >, determined to be greater than the value shown]

\begin{tabular}{|c|c|c|c|c|c|c|c|c|c|c|}
\hline Sample & Latitude & Longitude & Ag ppm-ICP & As ppm-ICP & Au ppm-ICP & Bi ppm-ICP & Cd ppm-ICP & Cu ppm-ICP & Mo ppm-ICP & Pb ppm-IC \\
\hline $\begin{array}{l}\text { HM0001S } \\
\text { HM0002S } \\
\text { HM0003S } \\
\text { HM0004S } \\
\text { HM0005S } \\
\text { HM0006S } \\
\text { HM0007S } \\
\text { HM0008S } \\
\text { HM0009S } \\
\text { HM0010S }\end{array}$ & $\begin{array}{llr}61 & 43 & 55 \\
61 & 42 & 59 \\
61 & 42 & 1 \\
61 & 39 & 48 \\
61 & 38 & 54 \\
61 & 38 & 9 \\
61 & 38 & 8 \\
61 & 36 & 35 \\
61 & 37 & 1 \\
61 & 41 & 25\end{array}$ & $\begin{array}{rrr}158 & 29 & 30 \\
158 & 29 & 58 \\
158 & 30 & 32 \\
158 & 34 & 5 \\
158 & 35 & 38 \\
158 & 31 & 45 \\
158 & 31 & 32 \\
158 & 24 & 37 \\
158 & 24 & 0 \\
158 & 26 & 26\end{array}$ & $\begin{array}{l}.13 \\
.24 \\
.12 \\
.18 \\
.072 \\
N \\
.59 \\
N \\
N \\
.68\end{array}$ & $\begin{array}{c}180 \\
280 \\
360 \\
150 \\
5.4 \\
8 \\
210 \\
3.8 \\
9.1 \\
140\end{array}$ & $\begin{array}{l}N \\
\dot{N}^{14} \\
N \\
N \\
N \\
N \\
N \\
N \\
N\end{array}$ & $\begin{array}{l}1.3 \\
.74 \\
N \\
N \\
N \\
N \\
1.9 \\
N \\
N \\
N\end{array}$ & $\begin{array}{l}.3 \\
.28 \\
.28 \\
.56 \\
.15 \\
.097 \\
.14 \\
.11 \\
.21 \\
1.9\end{array}$ & $\begin{array}{c}35 \\
32 \\
26 \\
42 \\
12 \\
6.6 \\
120 \\
10 \\
12 \\
30\end{array}$ & $\begin{array}{l}1.1 \\
1.2 \\
1.9 \\
1.4 \\
.35 \\
.42 \\
.76 \\
.25 \\
.74 \\
.84\end{array}$ & $\begin{array}{l}13 \\
15 \\
16 \\
14 \\
8.1 \\
5.3 \\
16 \\
5.6 \\
9.1 \\
54\end{array}$ \\
\hline $\begin{array}{l}\text { HM0011S } \\
\text { HMO012S } \\
\text { HM0013S } \\
\text { HM0014S } \\
\text { HMO015S } \\
\text { HMO016S } \\
\text { HM0017S } \\
\text { HMO018S } \\
\text { HMO019S } \\
\text { HMO020S }\end{array}$ & $\begin{array}{lll}61 & 41 & 22 \\
61 & 42 & 20 \\
61 & 44 & 0 \\
61 & 45 & 46 \\
61 & 45 & 44 \\
61 & 47 & 13 \\
61 & 47 & 43 \\
61 & 41 & 27 \\
61 & 44 & 27 \\
61 & 45 & 21\end{array}$ & $\begin{array}{lll}158 & 16 & 41 \\
158 & 24 & 35 \\
158 & 18 & 56 \\
158 & 20 & 17 \\
158 & 20 & 16 \\
158 & 24 & 15 \\
158 & 25 & 33 \\
158 & 36 & 46 \\
158 & 35 & 51 \\
158 & 36 & 40\end{array}$ & $\begin{array}{l}N \\
N \\
N \\
N \\
N \\
.14 \\
._{N}^{95} \\
.091\end{array}$ & $\begin{array}{c}4.2 \\
14 \\
2.9 \\
18 \\
18 \\
180 \\
120 \\
10 \\
30 \\
12\end{array}$ & $\begin{array}{l}N \\
N \\
N \\
N \\
N \\
N \\
N \\
N \\
N \\
N\end{array}$ & $\begin{array}{l}N \\
N \\
N \\
N \\
N \\
1.7 \\
5 \\
N \\
N \\
N\end{array}$ & $\begin{array}{l}.15 \\
.15 \\
.14 \\
.13 \\
.16 \\
.43 \\
1.5 \\
.17 \\
.29 \\
.16\end{array}$ & $\begin{array}{l}12 \\
15 \\
16 \\
13 \\
14 \\
18 \\
33 \\
9.1 \\
18 \\
14\end{array}$ & $\begin{array}{c}.32 \\
1.6 \\
.43 \\
.93 \\
1.1 \\
1.5 \\
.92 \\
.62 \\
.8 \\
.79\end{array}$ & $\begin{array}{c}5.6 \\
12 \\
7.6 \\
9.1 \\
11 \\
17 \\
56 \\
6.5 \\
11 \\
11\end{array}$ \\
\hline $\begin{array}{l}\text { HM0021S } \\
\text { HM0022S } \\
\text { HM0023S } \\
\text { HM0024S } \\
\text { HM0025S } \\
\text { HM0026S } \\
\text { HM0027S } \\
\text { HM0028S } \\
\text { HM0029S } \\
\text { HM0030S }\end{array}$ & $\begin{array}{rrr}61 & 46 & 13 \\
61 & 46 & 9 \\
61 & 49 & 39 \\
61 & 46 & 57 \\
61 & 51 & 31 \\
61 & 49 & 10 \\
61 & 53 & 12 \\
61 & 54 & 7 \\
61 & 53 & 53 \\
61 & 56 & 11\end{array}$ & $\begin{array}{rrr}158 & 33 & 24 \\
158 & 33 & 30 \\
158 & 26 & 24 \\
158 & 21 & 45 \\
158 & 18 & 5 \\
158 & 30 & 40 \\
158 & 28 & 40 \\
158 & 22 & 5 \\
158 & 16 & 37 \\
158 & 14 & 31\end{array}$ & $\begin{array}{l}N \\
.11 \\
.2 \\
N \\
N \\
N \\
N \\
N \\
N \\
N\end{array}$ & $\begin{array}{c}25 \\
20 \\
120 \\
25 \\
3 \\
27 \\
.98 \\
4.6 \\
5.8 \\
N\end{array}$ & $\begin{array}{l}N \\
N \\
N \\
N \\
N \\
N \\
N \\
N \\
N \\
N\end{array}$ & $\begin{array}{l}N \\
.68 \\
2.2 \\
N \\
N \\
N \\
N \\
N \\
N \\
N\end{array}$ & $\begin{array}{l}.25 \\
.35 \\
.79 \\
.18 \\
.1 \\
.15 \\
.078 \\
.14 \\
.23 \\
.11\end{array}$ & $\begin{array}{c}20 \\
17 \\
20 \\
12 \\
11 \\
15 \\
6.1 \\
8.4 \\
16 \\
8.8\end{array}$ & $\begin{array}{l}.69 \\
.95 \\
1.3 \\
.9 \\
.34 \\
.56 \\
.37 \\
.31 \\
.48 \\
.3\end{array}$ & $\begin{array}{l}11 \\
13 \\
30 \\
9.3 \\
7.2 \\
8.9 \\
6.4 \\
7.5 \\
9.3 \\
5.8\end{array}$ \\
\hline $\begin{array}{l}\text { HMO031S } \\
\text { HMO032S } \\
\text { HMO033S } \\
\text { HM0034S } \\
\text { HM0035S } \\
\text { HMO036S } \\
\text { HM0037S } \\
\text { HMO038S } \\
\text { HMO039S } \\
\text { HMO040S }\end{array}$ & $\begin{array}{lll}61 & 50 & 56 \\
61 & 48 & 47 \\
61 & 47 & 21 \\
61 & 45 & 57 \\
61 & 55 & 49 \\
61 & 55 & 48 \\
61 & 59 & 19 \\
61 & 57 & 15 \\
61 & 59 & 16 \\
61 & 55 & 36\end{array}$ & $\begin{array}{rrr}158 & 11 & 41 \\
158 & 7 & 21 \\
158 & 15 & 17 \\
158 & 15 & 48 \\
158 & 50 & 5 \\
158 & 58 & 38 \\
158 & 47 & 3 \\
158 & 40 & 8 \\
158 & 34 & 58 \\
158 & 36 & 1\end{array}$ & $\begin{array}{l}N \\
N \\
N \\
N \\
N \\
N \\
N \\
i_{N}^{14}\end{array}$ & $\begin{array}{c}N \\
4.3 \\
N \\
2.9 \\
24 \\
5.3 \\
8.3 \\
4.8 \\
4.7 \\
8.6\end{array}$ & $\begin{array}{l}N \\
N \\
N \\
N \\
N \\
N \\
N \\
N \\
N \\
N\end{array}$ & $\begin{array}{l}N \\
N \\
N \\
N \\
N \\
N \\
N \\
N \\
N \\
N\end{array}$ & $\begin{array}{l}.088 \\
.11 \\
.11 \\
.14 \\
.15 \\
.14 \\
.11 \\
.27 \\
.099 \\
.16\end{array}$ & $\begin{array}{l}9.5 \\
10 \\
12 \\
11 \\
15 \\
9.2 \\
11 \\
14 \\
14 \\
7.7\end{array}$ & $\begin{array}{l}.42 \\
.35 \\
.35 \\
.26 \\
.81 \\
.41 \\
.54 \\
.61 \\
.55 \\
.42\end{array}$ & $\begin{array}{c}5.6 \\
4.5 \\
5.7 \\
5.6 \\
6.6 \\
4.7 \\
6.5 \\
11 \\
6.6 \\
9.8\end{array}$ \\
\hline $\begin{array}{l}\text { HM0041S } \\
\text { HM0042S } \\
\text { HM0043S } \\
\text { HM0044S } \\
\text { HM0045S } \\
\text { HM0046S } \\
\text { HM0047S } \\
\text { HM0048S } \\
\text { HM0049S } \\
\text { HM0050S }\end{array}$ & $\begin{array}{rrr}61 & 59 & 0 \\
61 & 51 & 25 \\
61 & 53 & 42 \\
61 & 51 & 52 \\
61 & 47 & 45 \\
61 & 45 & 3 \\
61 & 51 & 40 \\
61 & 43 & 44 \\
61 & 39 & 55 \\
61 & 40 & 6\end{array}$ & $\begin{array}{rrr}158 & 18 & 44 \\
158 & 38 & 19 \\
158 & 55 & 25 \\
158 & 45 & 54 \\
158 & 50 & 32 \\
158 & 55 & 1 \\
158 & 59 & 9 \\
158 & 59 & 17 \\
158 & 48 & 41 \\
158 & 45 & 55\end{array}$ & $\begin{array}{l}N \\
N \\
.1 \\
.072 \\
N \\
N \\
N \\
.095 \\
N \\
N\end{array}$ & $\begin{array}{l}6.2 \\
5 \\
4.7 \\
N \\
6 \\
7.1 \\
8.3 \\
6.1 \\
5.7 \\
18\end{array}$ & $\begin{array}{l}N \\
N \\
N \\
N \\
N \\
N \\
N \\
N \\
N \\
N\end{array}$ & $\begin{array}{l}N \\
N \\
N \\
N \\
N \\
N \\
N \\
N \\
N \\
N\end{array}$ & $\begin{array}{l}.098 \\
.14 \\
.24 \\
.28 \\
.077 \\
.1 \\
.095 \\
.22 \\
.081 \\
.14\end{array}$ & $\begin{array}{l}13 \\
9.2 \\
14 \\
14 \\
15 \\
15 \\
14 \\
19 \\
14 \\
18\end{array}$ & $\begin{array}{l}.66 \\
.17 \\
.51 \\
.41 \\
.76 \\
.81 \\
.46 \\
1 \\
.5 \\
.55\end{array}$ & $\begin{array}{l}6.8 \\
7 \\
8.3 \\
8.1 \\
7.1 \\
7.3 \\
7.2 \\
8.2 \\
5.5 \\
6.2\end{array}$ \\
\hline $\begin{array}{l}\text { HMO100S } \\
\text { HMO101S } \\
\text { HM0102S } \\
\text { HM0103S } \\
\text { HM0104S } \\
\text { HM0105S } \\
\text { HM0106S } \\
\text { HM0107S } \\
\text { HM0108S } \\
\text { HM0109S }\end{array}$ & $\begin{array}{rrr}61 & 43 & 56 \\
61 & 44 & 18 \\
61 & 41 & 7 \\
61 & 38 & 19 \\
61 & 37 & 24 \\
61 & 36 & 49 \\
61 & 38 & 11 \\
61 & 41 & 6 \\
61 & 40 & 4 \\
61 & 43 & 6\end{array}$ & $\begin{array}{lll}158 & 29 & 19 \\
158 & 28 & 15 \\
158 & 31 & 18 \\
158 & 34 & 12 \\
158 & 29 & 28 \\
158 & 28 & 30 \\
158 & 25 & 7 \\
158 & 28 & 10 \\
158 & 19 & 13 \\
158 & 23 & 27\end{array}$ & $\begin{array}{l}.14 \\
.15 \\
.08 \\
.084 \\
N \\
N \\
N \\
N \\
N \\
.11\end{array}$ & $\begin{array}{c}370 \\
340 \\
210 \\
15 \\
11 \\
5.7 \\
5.6 \\
13 \\
7.2 \\
18\end{array}$ & $\begin{array}{l}N \\
N \\
\dot{N} \\
N \\
N \\
N \\
N \\
N \\
N\end{array}$ & $\begin{array}{l}.96 \\
1.5 \\
N \\
N \\
N \\
N \\
N \\
N \\
N\end{array}$ & $\begin{array}{l}.34 \\
.24 \\
.19 \\
.13 \\
.086 \\
.11 \\
.097 \\
.13 \\
.14 \\
.28\end{array}$ & $\begin{array}{l}28 \\
39 \\
19 \\
6.5 \\
20 \\
10 \\
14 \\
14 \\
20 \\
15\end{array}$ & $\begin{array}{l}1.8 \\
1.6 \\
1.3 \\
.23 \\
.9 \\
.44 \\
.47 \\
.63 \\
.71 \\
1.1\end{array}$ & $\begin{array}{l}14 \\
11 \\
12 \\
6 \\
8.9 \\
7 \\
5.6 \\
11 \\
7.1 \\
9.3\end{array}$ \\
\hline $\begin{array}{l}\text { HMO110S } \\
\text { HMO111S } \\
\text { HMO112S } \\
\text { HMO113S } \\
\text { HMO114S }\end{array}$ & $\begin{array}{rrr}61 & 43 & 1 \\
61 & 44 & 55 \\
61 & 45 & 59 \\
61 & 48 & 35 \\
61 & 39 & 56\end{array}$ & $\begin{array}{rrr}158 & 17 & 45 \\
158 & 21 & 58 \\
158 & 25 & 43 \\
158 & 19 & 4 \\
158 & 36 & 13\end{array}$ & $\begin{array}{l}N \\
N \\
.26 \\
N \\
N\end{array}$ & $\begin{array}{c}8.3 \\
38 \\
290 \\
3.7 \\
29\end{array}$ & $\begin{array}{l}N \\
N \\
N \\
N \\
N\end{array}$ & $\begin{array}{c}N \\
N \\
2.2 \\
N \\
N\end{array}$ & $\begin{array}{l}.17 \\
.4 \\
.45 \\
.078 \\
.21\end{array}$ & $\begin{array}{c}20 \\
17 \\
25 \\
12 \\
8.3\end{array}$ & $\begin{array}{l}1 \\
.96 \\
1.4 \\
.42 \\
.84\end{array}$ & $\begin{array}{l}8.2 \\
13 \\
21 \\
7.1 \\
9.1\end{array}$ \\
\hline
\end{tabular}


Table 3. Analytical data for stream-sediment samples collected from the Horn Mountains area--Continued.

\begin{tabular}{|c|c|c|c|c|c|c|c|c|c|c|}
\hline Sample & Sb ppm-ICP & Zn ppm-ICP & Ca X-sas & Fe $x$-sas & Mg $\%$-sQS & Na \%-Sas & $P \%$-sas & Ti X-sQS & Ag ppm-sos As & ppm-SQS \\
\hline $\begin{array}{l}\text { HMO001s } \\
\text { HMO002S } \\
\text { HM0003S } \\
\text { HMO004S } \\
\text { HMO005S } \\
\text { HMO006S } \\
\text { HMO0075 } \\
\text { HMO008S } \\
\text { HMO009S } \\
\text { HMO010S }\end{array}$ & $\begin{array}{c}6.9 \\
12 \\
7.2 \\
27^{N} \\
N 5^{N} \\
2^{N}\end{array}$ & $\begin{array}{l}67 \\
76 \\
71 \\
96 \\
64 \\
49 \\
87 \\
53 \\
66 \\
150\end{array}$ & $\begin{array}{l}.15 \\
.1 \\
.15 \\
.2 \\
.1 \\
.15 \\
.15 \\
.15 \\
.1 \\
.2\end{array}$ & $\begin{array}{l}3 \\
2 \\
3 \\
7 \\
2 \\
2 \\
5 \\
2 \\
5 \\
5\end{array}$ & $\begin{array}{l}.5 \\
.2 \\
.5 \\
.5 \\
.2 \\
.2 \\
.5 \\
.3 \\
.5\end{array}$ & $\begin{array}{l}2 \\
1.5 \\
2 \\
1.5 \\
2 \\
2 \\
1.5 \\
3 \\
2 \\
2\end{array}$ & $\begin{array}{l}N \\
N \\
N \\
N \\
N \\
N \\
N \\
N \\
N \\
N\end{array}$ & $\begin{array}{l}.3 \\
.5 \\
.5 \\
.15 \\
.5 \\
.3 \\
.2 \\
.5 \\
.5\end{array}$ & $\begin{array}{l}N \\
N \\
N \\
N \\
N \\
N \\
.5 \\
N \\
N \\
<.5\end{array}$ & $\begin{array}{l}N \\
N \\
N \\
N \\
N \\
N \\
N \\
N \\
N \\
N\end{array}$ \\
\hline $\begin{array}{l}\text { HMO011S } \\
\text { HM0012S } \\
\text { HM0013S } \\
\text { HM0014S } \\
\text { HM0015S } \\
\text { HM0016S } \\
\text { HM0017s } \\
\text { HMO018S } \\
\text { HMO019S } \\
\text { HMO020S }\end{array}$ & $\begin{array}{c}N \\
.95 \\
N \\
1.4 \\
1 \\
9.2 \\
35 \\
3.5 \\
.72\end{array}$ & $\begin{array}{r}52 \\
67 \\
88 \\
74 \\
93 \\
78 \\
170 \\
81 \\
70 \\
77\end{array}$ & $\begin{array}{l}.15 \\
.15 \\
.1 \\
.1 \\
.2 \\
.2 \\
.2 \\
.15 \\
.2 \\
.2\end{array}$ & $\begin{array}{l}2 \\
3 \\
2 \\
3 \\
5 \\
5 \\
3 \\
3 \\
3 \\
5\end{array}$ & $\begin{array}{l}.3 \\
.5 \\
.3 \\
.3 \\
1.5 \\
.5 \\
.5 \\
.5 \\
.3 \\
.7\end{array}$ & $\begin{array}{l}2 \\
3 \\
1 \\
3 \\
3 \\
3 \\
3 \\
2 \\
3 \\
2\end{array}$ & $\begin{array}{l}N \\
N \\
N \\
N \\
N \\
N \\
N \\
N \\
N \\
N\end{array}$ & $\begin{array}{l}.3 \\
.5 \\
.2 \\
.5 \\
1.5 \\
.5 \\
.5 \\
.5 \\
. .5\end{array}$ & $\begin{array}{l}N \\
N \\
N \\
N \\
N \\
N \\
.5 \\
N \\
N \\
N\end{array}$ & $\begin{array}{l}N \\
N \\
N \\
N \\
N \\
N \\
N \\
N \\
N \\
N\end{array}$ \\
\hline $\begin{array}{l}\text { HMO021S } \\
\text { HMO022S } \\
\text { HMO023S } \\
\text { HM0024S } \\
\text { HMO025S } \\
\text { HMO026S } \\
\text { HM0027S } \\
\text { HM0028S } \\
\text { HM0029S } \\
\text { HMO03OS }\end{array}$ & $\begin{array}{c}1.5 \\
4 \\
15 \\
7 \\
N \\
1.4 \\
N \\
N \\
N \\
N\end{array}$ & $\begin{array}{r}89 \\
65 \\
110 \\
66 \\
67 \\
81 \\
41 \\
57 \\
65 \\
64\end{array}$ & $\begin{array}{l}.5 \\
.3 \\
.2 \\
.2 \\
.15 \\
.5 \\
.15 \\
.2 \\
.2 \\
.15\end{array}$ & $\begin{array}{l}3 \\
5 \\
3 \\
5 \\
3 \\
5 \\
2 \\
2 \\
3 \\
5\end{array}$ & $\begin{array}{r}1.5 \\
.5 \\
.5 \\
.7 \\
.5 \\
1.5 \\
.2 \\
.2 \\
.5 \\
.3\end{array}$ & $\begin{array}{l}3 \\
2 \\
2 \\
5 \\
3 \\
2 \\
3 \\
3 \\
2 \\
3\end{array}$ & $\begin{array}{l}N \\
N \\
N \\
N \\
N \\
N \\
N \\
N \\
N \\
N\end{array}$ & $\begin{array}{l}.5 \\
.7 \\
.3 \\
1.3 \\
.3 \\
.7 \\
.5 \\
.5 \\
.3 \\
.3\end{array}$ & $\begin{array}{l}N \\
N \\
N \\
N \\
N \\
N \\
N \\
N \\
N \\
N\end{array}$ & $\begin{array}{l}N \\
N \\
N \\
N \\
N \\
N \\
N \\
N \\
N \\
N\end{array}$ \\
\hline $\begin{array}{l}\text { HMO031S } \\
\text { HM0032S } \\
\text { HM0033S } \\
\text { HM0034S } \\
\text { HMO035S } \\
\text { HMO036S } \\
\text { HM0037S } \\
\text { HMO038S } \\
\text { HMO039S } \\
\text { HM0040S }\end{array}$ & $\begin{array}{c}N \\
N \\
N \\
1.3 \\
N \\
N \\
N \\
N \\
1.7\end{array}$ & $\begin{array}{l}60 \\
56 \\
58 \\
61 \\
92 \\
66 \\
77 \\
66 \\
81 \\
45\end{array}$ & $\begin{array}{l}.07 \\
.15 \\
.1 \\
.1 \\
.05 \\
.05 \\
.15 \\
.15 \\
.1 \\
.3\end{array}$ & $\begin{array}{l}2 \\
2 \\
1.5 \\
2 \\
5 \\
2 \\
5 \\
3 \\
3 \\
5\end{array}$ & $\begin{array}{l}.3 \\
.5 \\
.3 \\
.5 \\
.3 \\
.2 \\
.5 \\
.2 \\
.3 \\
.7\end{array}$ & $\begin{array}{l}2 \\
1.5 \\
3 \\
1.5 \\
1.5 \\
1.5 \\
1.5 \\
2 \\
2 \\
.2\end{array}$ & $\begin{array}{l}N \\
N \\
N \\
N \\
N \\
N \\
N \\
N \\
N \\
N\end{array}$ & $\begin{array}{l}.3 \\
.3 \\
.2 \\
.2 \\
.15 \\
.2 \\
.5 \\
.2 \\
.5 \\
1\end{array}$ & $\begin{array}{l}N \\
N \\
N \\
N \\
N \\
N \\
N \\
N \\
N \\
N\end{array}$ & $\begin{array}{l}N \\
N \\
N \\
N \\
N \\
N \\
N \\
N \\
N \\
N\end{array}$ \\
\hline $\begin{array}{l}\text { HMO041S } \\
\text { HM0042S } \\
\text { HM0043S } \\
\text { HM0044S } \\
\text { HM0045S } \\
\text { HM0046S } \\
\text { HM0047S } \\
\text { HMO048S } \\
\text { HM0049S } \\
\text { HM0050S }\end{array}$ & $\begin{array}{l}1_{N}^{N} \\
.8 \\
\dot{N}^{N} \\
.^{N} \\
1^{N}\end{array}$ & $\begin{array}{l}77 \\
56 \\
64 \\
63 \\
89 \\
91 \\
81 \\
99 \\
88 \\
84\end{array}$ & $\begin{array}{l}.05 \\
.15 \\
.15 \\
.15 \\
.1 \\
.07 \\
.15 \\
.05 \\
.05 \\
.05\end{array}$ & $\begin{array}{l}2 \\
3 \\
3 \\
3 \\
3 \\
5 \\
3 \\
3 \\
3 \\
3\end{array}$ & $\begin{array}{l}.2 \\
.3 \\
.2 \\
.3 \\
.5 \\
.3 \\
.5 \\
.3 \\
.7 \\
.3\end{array}$ & $\begin{array}{l}1.5 \\
2 \\
2 \\
2 \\
3 \\
2 \\
1.5 \\
1.5 \\
1.5 \\
1.5\end{array}$ & $\begin{array}{l}N \\
N \\
N \\
N \\
N \\
N \\
N \\
N \\
N \\
N\end{array}$ & $\begin{array}{l}.2 \\
.5 \\
.3 \\
.3 \\
.2 \\
.3 \\
.5 \\
.2 \\
.3 \\
.3\end{array}$ & $\begin{array}{l}N \\
N \\
N \\
N \\
N \\
N \\
N \\
N \\
N \\
N\end{array}$ & $\begin{array}{l}N \\
N \\
N \\
N \\
N \\
N \\
N \\
N \\
N\end{array}$ \\
\hline $\begin{array}{l}\text { HM0100S } \\
\text { HM0101S } \\
\text { HM0102S } \\
\text { HM0103S } \\
\text { HM0104S } \\
\text { HM0105S } \\
\text { HM0106S } \\
\text { HM0107S } \\
\text { HM0108S } \\
\text { HM0109S }\end{array}$ & $\begin{array}{l}12 \\
17 \\
6.3 \\
1.4 \\
N \\
.71 \\
N \\
2.3 \\
N \\
N\end{array}$ & $\begin{array}{l}64 \\
56 \\
60 \\
43 \\
96 \\
74 \\
71 \\
89 \\
96 \\
67\end{array}$ & $\begin{array}{l}.1 \\
.15 \\
.1 \\
.1 \\
.15 \\
.1 \\
.07 \\
.15 \\
.05 \\
.2\end{array}$ & $\begin{array}{l}1.5 \\
3 \\
2 \\
2 \\
5 \\
3 \\
2 \\
3 \\
5 \\
3\end{array}$ & $\begin{array}{l}.2 \\
.3 \\
.3 \\
.3 \\
.5 \\
.3 \\
.3 \\
.5 \\
.5 \\
.5\end{array}$ & $\begin{array}{l}2 \\
3 \\
3 \\
2 \\
1.5 \\
2 \\
1.5 \\
3 \\
1 \\
3\end{array}$ & $\begin{array}{l}N \\
N \\
N \\
N \\
N \\
N \\
N \\
N \\
N \\
N\end{array}$ & $\begin{array}{l}.3 \\
.2 \\
.5 \\
.7 \\
.3 \\
.3 \\
.2 \\
.3 \\
.3 \\
.3\end{array}$ & $\begin{array}{l}N \\
N \\
N \\
N \\
<.5 \\
N \\
N \\
N \\
N \\
N\end{array}$ & $\begin{array}{l}N \\
N \\
N \\
N \\
N \\
N \\
N \\
N \\
N \\
N\end{array}$ \\
\hline $\begin{array}{l}\text { HMO110S } \\
\text { HM0111S } \\
\text { HM0112S } \\
\text { HM0113S } \\
\text { HM0114S }\end{array}$ & $\begin{array}{c}5^{N} \\
19 \\
1.4\end{array}$ & $\begin{array}{l}97 \\
73 \\
86 \\
74 \\
57\end{array}$ & $\begin{array}{l}.07 \\
.15 \\
.2 \\
.1 \\
.2\end{array}$ & $\begin{array}{l}3 \\
3 \\
5 \\
2 \\
3\end{array}$ & $\begin{array}{l}.3 \\
.3 \\
.5 \\
.3 \\
.3\end{array}$ & $\begin{array}{l}1.5 \\
3 \\
2 \\
3 \\
3\end{array}$ & $\begin{array}{l}N \\
N \\
N \\
N \\
N\end{array}$ & $\begin{array}{l}.3 \\
.5 \\
.5 \\
.2 \\
.3\end{array}$ & $\begin{array}{l}N \\
N \\
N \\
N \\
N\end{array}$ & $\begin{array}{r}N \\
N \\
N \\
N \\
N\end{array}$ \\
\hline
\end{tabular}


Table 3. Analytical data for stream-sediment samples collected from the Horn Mountains area--Continued.

\begin{tabular}{|c|c|c|c|c|c|c|c|c|c|c|}
\hline Sample & Au ppm-SQS & B ppm-sas & Ba ppm-SQS & Be ppm-sas & Bi ppm-SQS & Cd ppm-sos & Co ppm-SQS & Cr ppm-sQs & Cu ppm-SQS Ge & Ga ppm-sa \\
\hline $\begin{array}{l}\text { HM0001S } \\
\text { HM0002S } \\
\text { HM0003S } \\
\text { HM0004S } \\
\text { HM0005S } \\
\text { HM0006S } \\
\text { HM0007S } \\
\text { HMO008S } \\
\text { HM0009S } \\
\text { HM0010S }\end{array}$ & $\begin{array}{l}N \\
N \\
N \\
N \\
N \\
N \\
N \\
N \\
N \\
N\end{array}$ & $\begin{array}{r}70 \\
150 \\
50 \\
150 \\
<10 \\
70 \\
15 \\
N \\
15 \\
<10\end{array}$ & $\begin{array}{r}500 \\
300 \\
300 \\
1,000 \\
300 \\
700 \\
700 \\
300 \\
500 \\
300\end{array}$ & $\begin{array}{r}<1 \\
N \\
<1 \\
<1 \\
N \\
<1 \\
N \\
N \\
N \\
1\end{array}$ & $\begin{array}{l}N \\
N \\
N \\
N \\
N \\
N \\
N \\
N \\
N \\
N\end{array}$ & $\begin{array}{l}N \\
N \\
N \\
N \\
N \\
N \\
N \\
N \\
N \\
N\end{array}$ & $\begin{array}{r}10 \\
<10 \\
10 \\
30 \\
10 \\
<10 \\
20 \\
<10 \\
15 \\
20\end{array}$ & $\begin{array}{r}100 \\
70 \\
100 \\
500 \\
30 \\
100 \\
300 \\
50 \\
200 \\
500\end{array}$ & $\begin{array}{r}50 \\
30 \\
50 \\
100 \\
10 \\
15 \\
150 \\
10 \\
20 \\
50\end{array}$ & $\begin{array}{r}30 \\
7 \\
20 \\
20 \\
15 \\
20 \\
15 \\
30 \\
30 \\
20\end{array}$ \\
\hline $\begin{array}{l}\text { HMO011S } \\
\text { HM0012S } \\
\text { HMO013S } \\
\text { HM0014S } \\
\text { HM0015S } \\
\text { HM0016S } \\
\text { HM0017S } \\
\text { HM0018S } \\
\text { HM0019S } \\
\text { HMO020S }\end{array}$ & $\begin{array}{l}N \\
N \\
N \\
N \\
N \\
N \\
N \\
N \\
N \\
N\end{array}$ & $\begin{array}{r}10 \\
N \\
10 \\
N \\
10 \\
100 \\
100 \\
10 \\
<10 \\
<10\end{array}$ & $\begin{array}{l}500 \\
500 \\
300 \\
300 \\
500 \\
500 \\
500 \\
500 \\
300 \\
500\end{array}$ & $\begin{array}{r}N \\
N \\
N \\
N \\
N \\
<1 \\
<1 \\
N \\
N \\
<1\end{array}$ & $\begin{array}{l}N \\
N \\
N \\
N \\
N \\
N \\
N \\
N \\
N \\
N\end{array}$ & $\begin{array}{l}N \\
N \\
N \\
N \\
N \\
N \\
N \\
N \\
N \\
N\end{array}$ & $\begin{array}{r}<10 \\
10 \\
10 \\
10 \\
30 \\
10 \\
15 \\
10 \\
<10 \\
20\end{array}$ & $\begin{array}{r}100 \\
200 \\
50 \\
200 \\
700 \\
200 \\
200 \\
100 \\
50 \\
500\end{array}$ & $\begin{array}{l}15 \\
30 \\
15 \\
20 \\
50 \\
30 \\
70 \\
15 \\
30 \\
30\end{array}$ & $\begin{array}{r}20 \\
30 \\
7 \\
20 \\
50 \\
30 \\
50 \\
20 \\
30 \\
30\end{array}$ \\
\hline $\begin{array}{l}\text { HM0021S } \\
\text { HMO022S } \\
\text { HM0023S } \\
\text { HM0024S } \\
\text { HM0025S } \\
\text { HM0026S } \\
\text { HM00275 } \\
\text { HM0028S } \\
\text { HM0029S } \\
\text { HM0030S }\end{array}$ & $\begin{array}{l}N \\
N \\
N \\
N \\
N \\
N \\
N \\
N \\
N \\
N\end{array}$ & $\begin{array}{r}N \\
15 \\
100 \\
<10 \\
<10 \\
15 \\
N \\
N \\
10 \\
10\end{array}$ & $\begin{array}{r}500 \\
500 \\
500 \\
500 \\
300 \\
500 \\
2,000 \\
1,000 \\
500 \\
300\end{array}$ & $\begin{array}{l}<1 \\
<1 \\
N \\
N \\
N \\
N \\
N \\
N \\
N \\
N\end{array}$ & $\begin{array}{l}N \\
N \\
N \\
N \\
N \\
N \\
N \\
N \\
N \\
N\end{array}$ & $\begin{array}{l}N \\
N \\
N \\
N \\
N \\
N \\
N \\
N \\
N \\
N\end{array}$ & $\begin{array}{r}15 \\
15 \\
15 \\
10 \\
15 \\
30 \\
<10 \\
<10 \\
20 \\
15\end{array}$ & $\begin{array}{r}500 \\
150 \\
300 \\
500 \\
300 \\
1,000 \\
200 \\
200 \\
100 \\
300\end{array}$ & $\begin{array}{r}20 \\
30 \\
30 \\
50 \\
7 \\
30 \\
7 \\
7 \\
20 \\
15\end{array}$ & $\begin{array}{l}30 \\
30 \\
20 \\
70 \\
30 \\
50 \\
30 \\
30 \\
30 \\
30\end{array}$ \\
\hline $\begin{array}{l}\text { HMO031S } \\
\text { HM0032S } \\
\text { HM0033S } \\
\text { HM0034S } \\
\text { HMO035S } \\
\text { HM0036S } \\
\text { HM0037S } \\
\text { HM0038S } \\
\text { HM0039S } \\
\text { HM0040S }\end{array}$ & $\begin{array}{l}N \\
N \\
N \\
N \\
N \\
N \\
N \\
N \\
N\end{array}$ & $\begin{array}{r}N \\
10 \\
N \\
10 \\
<10 \\
<10 \\
15 \\
10 \\
10 \\
10\end{array}$ & $\begin{array}{l}200 \\
500 \\
300 \\
300 \\
500 \\
300 \\
500 \\
500 \\
500 \\
700\end{array}$ & $\begin{array}{c}N \\
N \\
N \\
N \\
N \\
N \\
N \\
N \\
N \\
N \\
<1\end{array}$ & $\begin{array}{l}N \\
N \\
N \\
N \\
N \\
N \\
N \\
N \\
N \\
N\end{array}$ & $\begin{array}{l}N \\
N \\
N \\
N \\
N \\
N \\
N \\
N \\
N \\
N\end{array}$ & $\begin{array}{r}<10 \\
<10 \\
<10 \\
<10 \\
10 \\
15 \\
20 \\
15 \\
20 \\
15\end{array}$ & $\begin{array}{r}70 \\
50 \\
70 \\
100 \\
500 \\
300 \\
1,500 \\
50 \\
500 \\
1,000\end{array}$ & $\begin{array}{l}7 \\
15 \\
10 \\
20 \\
10 \\
15 \\
20 \\
15 \\
20 \\
20\end{array}$ & $\begin{array}{l}15 \\
20 \\
10 \\
10 \\
15 \\
15 \\
15 \\
15 \\
15 \\
20\end{array}$ \\
\hline $\begin{array}{l}\text { HMO041S } \\
\text { HMO042S } \\
\text { HM0043S } \\
\text { HM0044S } \\
\text { HM0045S } \\
\text { HM0046S } \\
\text { HMO047S } \\
\text { HM0048S } \\
\text { HM0049S } \\
\text { HM0050S }\end{array}$ & $\begin{array}{l}N \\
N \\
N \\
N \\
N \\
N \\
N \\
N \\
N \\
N\end{array}$ & $\begin{array}{r}<10 \\
10 \\
<10 \\
15 \\
N \\
10 \\
10 \\
10 \\
20 \\
10\end{array}$ & $\begin{array}{l}200 \\
500 \\
500 \\
700 \\
300 \\
300 \\
700 \\
300 \\
500 \\
500\end{array}$ & $\begin{array}{l}N \\
N \\
N \\
N \\
N \\
N \\
N \\
N \\
N \\
N\end{array}$ & $\begin{array}{l}N \\
N \\
N \\
N \\
N \\
N \\
N \\
N \\
N \\
N\end{array}$ & $\begin{array}{l}N \\
N \\
N \\
N \\
N \\
N \\
N \\
N \\
N \\
N\end{array}$ & $\begin{array}{l}10 \\
10 \\
10 \\
20 \\
20 \\
20 \\
15 \\
15 \\
15 \\
15\end{array}$ & $\begin{array}{r}500 \\
150 \\
70 \\
100 \\
200 \\
1,000 \\
500 \\
200 \\
200 \\
500\end{array}$ & $\begin{array}{l}10 \\
20 \\
20 \\
20 \\
20 \\
15 \\
15 \\
20 \\
20 \\
20\end{array}$ & $\begin{array}{l}10 \\
20 \\
30 \\
20 \\
30 \\
20 \\
15 \\
20 \\
30 \\
15\end{array}$ \\
\hline $\begin{array}{l}\text { HM0100S } \\
\text { HM0101S } \\
\text { HM0102S } \\
\text { HM0103S } \\
\text { HM0104S } \\
\text { HM0105S } \\
\text { HM0106S } \\
\text { HM0107S } \\
\text { HM0108S } \\
\text { HM0109S }\end{array}$ & $\begin{array}{l}N \\
N \\
N \\
N \\
N \\
N \\
N \\
N \\
N \\
N\end{array}$ & $\begin{array}{r}100 \\
100 \\
50 \\
30 \\
20 \\
15 \\
<10 \\
<10 \\
10 \\
<10\end{array}$ & $\begin{array}{l}300 \\
500 \\
300 \\
500 \\
700 \\
500 \\
200 \\
500 \\
300 \\
500\end{array}$ & $\begin{array}{l}<1 \\
N \\
N \\
N \\
N \\
N \\
N \\
N \\
N \\
N\end{array}$ & $\begin{array}{l}N \\
N \\
N \\
N \\
N \\
N \\
N \\
N \\
N \\
N\end{array}$ & $\begin{array}{l}N \\
N \\
N \\
N \\
N \\
N \\
N \\
N \\
N \\
N\end{array}$ & $\begin{array}{r}<10 \\
10 \\
<10 \\
<10 \\
20 \\
10 \\
15 \\
15 \\
20 \\
10\end{array}$ & $\begin{array}{r}50 \\
100 \\
200 \\
200 \\
500 \\
200 \\
150 \\
700 \\
150 \\
100\end{array}$ & $\begin{array}{l}30 \\
50 \\
30 \\
15 \\
50 \\
15 \\
10 \\
20 \\
30 \\
30\end{array}$ & $\begin{array}{l}15 \\
20 \\
20 \\
20 \\
30 \\
20 \\
15 \\
30 \\
30 \\
30\end{array}$ \\
\hline $\begin{array}{l}\text { HM0110S } \\
\text { HM0111S } \\
\text { HM0112S } \\
\text { HM0113S } \\
\text { HMO114S }\end{array}$ & $\begin{array}{l}N \\
N \\
N \\
N \\
N\end{array}$ & $\begin{array}{r}20 \\
<10 \\
70 \\
N \\
20\end{array}$ & $\begin{array}{l}300 \\
300 \\
500 \\
300 \\
700\end{array}$ & $\begin{array}{l}N \\
N \\
1 \\
N \\
N\end{array}$ & $\begin{array}{l}N \\
N \\
N \\
N \\
N\end{array}$ & $\begin{array}{l}N \\
N \\
N \\
N \\
N\end{array}$ & $\begin{array}{l}15 \\
10 \\
15 \\
10 \\
10\end{array}$ & $\begin{array}{r}150 \\
50 \\
100 \\
50 \\
100\end{array}$ & $\begin{array}{l}20 \\
20 \\
50 \\
10 \\
15\end{array}$ & $\begin{array}{l}20 \\
20 \\
50 \\
20 \\
30\end{array}$ \\
\hline
\end{tabular}


Table 3. Analytical data for stream-sediment samples collected from the Horn Mountains area--Continued.

\begin{tabular}{|c|c|c|c|c|c|c|c|c|c|}
\hline Sample & Ge ppm-Sas & La ppm-SQS & Mn ppm-SQS & Mo ppm-SQS & Nb ppm-SQS & Mi ppm-SQS & Pb ppm-sas & Sb ppm-SQS & Sc ppm-SQS \\
\hline $\begin{array}{l}\text { HM0001S } \\
\text { HMO002S } \\
\text { HM0003S } \\
\text { HM0004S } \\
\text { HMO005S } \\
\text { HMO006S } \\
\text { HM00075 } \\
\text { HMO008S } \\
\text { HM0009S } \\
\text { HMO010S }\end{array}$ & $\begin{array}{l}N \\
N \\
N \\
N \\
N \\
N \\
N \\
N \\
N \\
N\end{array}$ & $\begin{array}{l}N \\
N \\
N \\
N \\
N \\
N \\
N \\
N \\
N \\
N\end{array}$ & $\begin{array}{r}500 \\
300 \\
300 \\
1,000 \\
200 \\
200 \\
300 \\
100 \\
300 \\
500\end{array}$ & $\begin{array}{r}N \\
N \\
<5 \\
<5 \\
N \\
N \\
N \\
N \\
N \\
N \\
N\end{array}$ & $\begin{array}{l}N \\
N \\
N \\
N \\
N \\
N \\
N \\
N \\
N \\
N\end{array}$ & $\begin{array}{r}15 \\
7 \\
15 \\
70 \\
15 \\
10 \\
30 \\
10 \\
30 \\
100\end{array}$ & $\begin{array}{r}15 \\
10 \\
15 \\
10 \\
<10 \\
<10 \\
<10 \\
<10 \\
<10 \\
30\end{array}$ & $\begin{array}{l}N \\
N \\
N \\
N \\
N \\
N \\
N \\
N \\
N \\
N\end{array}$ & $\begin{array}{r}5 \\
<5 \\
5 \\
10 \\
<5 \\
<5 \\
5 \\
<5 \\
5 \\
7\end{array}$ \\
\hline $\begin{array}{l}\text { HM0011S } \\
\text { HM0012S } \\
\text { HM0013S } \\
\text { HM0014S } \\
\text { HM0015S } \\
\text { HM0016S } \\
\text { HM0017S } \\
\text { HM0018S } \\
\text { HMO019S } \\
\text { HM0020S }\end{array}$ & $\begin{array}{l}N \\
N \\
N \\
N \\
N \\
N \\
N \\
N \\
N \\
N\end{array}$ & $\begin{array}{l}N \\
N \\
N \\
N \\
N \\
N \\
N \\
N \\
N \\
N\end{array}$ & $\begin{array}{l}100 \\
200 \\
100 \\
150 \\
200 \\
200 \\
200 \\
700 \\
300 \\
300\end{array}$ & $\begin{array}{r}N \\
<5 \\
N \\
N \\
N \\
N \\
<5 \\
N \\
N \\
N \\
N \\
<5\end{array}$ & $\begin{array}{l}\mathbf{N} \\
\mathbf{N} \\
\mathbf{N} \\
\mathbf{N} \\
\mathbf{N} \\
\mathbf{N} \\
\mathbf{N} \\
\mathbf{N} \\
\mathbf{N} \\
\mathbf{N}\end{array}$ & $\begin{array}{l}20 \\
15 \\
30 \\
15 \\
70 \\
15 \\
20 \\
20 \\
10 \\
50\end{array}$ & $\begin{array}{r}N \\
10 \\
N \\
<10 \\
10 \\
10 \\
50 \\
<10 \\
10 \\
10\end{array}$ & $\begin{array}{l}N \\
N \\
N \\
N \\
N \\
N \\
N \\
N \\
N \\
N\end{array}$ & $\begin{array}{r}<5 \\
5 \\
<5 \\
<5 \\
7 \\
5 \\
5 \\
5 \\
<5 \\
10\end{array}$ \\
\hline $\begin{array}{l}\text { HM0021S } \\
\text { HM0022S } \\
\text { HM0023S } \\
\text { HM0024S } \\
\text { HM0025S } \\
\text { HM0026S } \\
\text { HM0027S } \\
\text { HM0028S } \\
\text { HM0029S } \\
\text { HM0030S }\end{array}$ & $\begin{array}{l}N \\
N \\
N \\
N \\
N \\
N \\
N \\
N \\
N \\
N\end{array}$ & $\begin{array}{r}N \\
N \\
N \\
N \\
N \\
N \\
N \\
100 \\
N \\
N\end{array}$ & $\begin{array}{r}300 \\
300 \\
300 \\
500 \\
100 \\
500 \\
70 \\
70 \\
500 \\
100\end{array}$ & $\begin{array}{l}N \\
N \\
N \\
N \\
N \\
N \\
N \\
N \\
N \\
N\end{array}$ & $\begin{array}{l}N \\
N \\
N \\
N \\
N \\
N \\
N \\
N \\
N \\
N\end{array}$ & $\begin{array}{r}15 \\
20 \\
30 \\
7 \\
15 \\
50 \\
7 \\
7 \\
20 \\
20\end{array}$ & $\begin{array}{r}10 \\
<10 \\
20 \\
15 \\
<10 \\
<10 \\
15 \\
20 \\
<10 \\
N\end{array}$ & $\begin{array}{l}N \\
N \\
N \\
N \\
N \\
N \\
N \\
N \\
N \\
N\end{array}$ & $\begin{array}{r}5 \\
7 \\
7 \\
5 \\
5 \\
10 \\
<5 \\
<5 \\
5 \\
<5\end{array}$ \\
\hline $\begin{array}{l}\text { HM0031S } \\
\text { HM0032S } \\
\text { HM0033S } \\
\text { HM0034S } \\
\text { HM0035S } \\
\text { HM0036S } \\
\text { HM0037S } \\
\text { HM0038S } \\
\text { HM0039S } \\
\text { HMO04OS }\end{array}$ & $\begin{array}{l}N \\
N \\
N \\
N \\
N \\
N \\
N \\
N \\
N \\
N\end{array}$ & $\begin{array}{r}N \\
<50 \\
N \\
N \\
N \\
N \\
N \\
N \\
N \\
N\end{array}$ & $\begin{array}{r}100 \\
500 \\
70 \\
150 \\
150 \\
100 \\
200 \\
150 \\
100 \\
300\end{array}$ & $\begin{array}{l}N \\
N \\
N \\
N \\
N \\
N \\
N \\
N \\
N \\
N\end{array}$ & $\begin{array}{l}N \\
N \\
N \\
N \\
N \\
N \\
N \\
N \\
N \\
N\end{array}$ & $\begin{array}{l}15 \\
15 \\
10 \\
20 \\
15 \\
15 \\
50 \\
15 \\
30 \\
15\end{array}$ & $\begin{array}{r}N \\
<10 \\
<10 \\
N \\
N \\
N \\
N \\
N \\
N \\
10\end{array}$ & $\begin{array}{l}N \\
N \\
N \\
N \\
N \\
N \\
N \\
N \\
N \\
N\end{array}$ & $\begin{array}{l}<5 \\
<5 \\
<5 \\
<5 \\
<5 \\
<5 \\
7 \\
5 \\
5 \\
7\end{array}$ \\
\hline $\begin{array}{l}\text { HM0041S } \\
\text { HM0042S } \\
\text { HM0043S } \\
\text { HM0044S } \\
\text { HM0045S } \\
\text { HM0046S } \\
\text { HM0047S } \\
\text { HM0048S } \\
\text { HM0049S } \\
\text { HM0050S }\end{array}$ & $\begin{array}{l}N \\
N \\
N \\
N \\
N \\
N \\
N \\
N \\
N \\
N\end{array}$ & $\begin{array}{r}N \\
N \\
N \\
<50 \\
N \\
N \\
N \\
N \\
N \\
N\end{array}$ & $\begin{array}{l}150 \\
200 \\
150 \\
200 \\
200 \\
200 \\
100 \\
100 \\
150 \\
70\end{array}$ & $\begin{array}{l}N \\
N \\
N \\
N \\
N \\
N \\
N \\
N \\
N \\
N\end{array}$ & $\begin{array}{l}N \\
N \\
N \\
N \\
N \\
N \\
N \\
N \\
N \\
N\end{array}$ & $\begin{array}{l}20 \\
10 \\
20 \\
20 \\
15 \\
30 \\
50 \\
30 \\
30 \\
50\end{array}$ & $\begin{array}{r}N \\
<10 \\
<10 \\
N \\
<10 \\
N \\
N \\
N \\
N \\
N\end{array}$ & $\begin{array}{l}N \\
N \\
N \\
N \\
N \\
N \\
N \\
N \\
N \\
N\end{array}$ & $\begin{array}{r}<5 \\
<5 \\
<5 \\
5 \\
5 \\
5 \\
<5 \\
5 \\
5 \\
<5 \\
<5\end{array}$ \\
\hline $\begin{array}{l}\text { HM0100S } \\
\text { HM0101S } \\
\text { HM0102S } \\
\text { HM0103S } \\
\text { HM0104S } \\
\text { HM0105S } \\
\text { HM0106S } \\
\text { HM0107S } \\
\text { HM0108S } \\
\text { HM0109S }\end{array}$ & $\begin{array}{l}N \\
N \\
N \\
N \\
N \\
N \\
N \\
N \\
N \\
N\end{array}$ & $\begin{array}{r}N \\
N \\
N \\
N \\
N \\
50 \\
N \\
N \\
N \\
N\end{array}$ & $\begin{array}{l}150 \\
150 \\
150 \\
300 \\
200 \\
150 \\
70 \\
200 \\
100 \\
150\end{array}$ & $\begin{array}{r}N \\
N \\
N \\
N \\
N \\
<5 \\
N \\
N \\
N \\
N \\
N \\
<5\end{array}$ & $\begin{array}{l}N \\
N \\
N \\
N \\
N \\
N \\
N \\
N \\
N \\
N\end{array}$ & $\begin{array}{r}10 \\
15 \\
7 \\
10 \\
70 \\
20 \\
20 \\
30 \\
50 \\
15\end{array}$ & $\begin{array}{r}10 \\
15 \\
10 \\
10 \\
N \\
<10 \\
N \\
10 \\
N \\
10\end{array}$ & $\begin{array}{l}N \\
N \\
N \\
N \\
N \\
N \\
N \\
N \\
N \\
N\end{array}$ & $\begin{array}{r}<5 \\
<5 \\
<5 \\
<5 \\
7 \\
7 \\
<5 \\
<5 \\
<5 \\
5 \\
5\end{array}$ \\
\hline $\begin{array}{l}\text { HM0110S } \\
\text { HM0111S } \\
\text { HM0112S } \\
\text { HM0113S } \\
\text { HM0114S }\end{array}$ & $\begin{array}{l}N \\
N \\
N \\
N \\
N\end{array}$ & $\begin{array}{l}N \\
N \\
N \\
N \\
N\end{array}$ & $\begin{array}{r}100 \\
200 \\
300 \\
50 \\
500\end{array}$ & $\begin{array}{r}N \\
N \\
<5 \\
N \\
N\end{array}$ & $\begin{array}{l}N \\
N \\
N \\
N \\
N\end{array}$ & $\begin{array}{r}50 \\
10 \\
20 \\
7 \\
15\end{array}$ & $\begin{array}{r}N \\
15 \\
15 \\
10 \\
<10\end{array}$ & $\begin{array}{l}N \\
N \\
N \\
N \\
N\end{array}$ & $\begin{array}{r}<5 \\
5 \\
7 \\
<5 \\
<5\end{array}$ \\
\hline
\end{tabular}


Table 3. Analytical deta for stream-sediment samples collected from the Horn Mountains area--Continued.

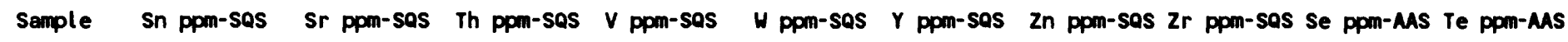

\begin{tabular}{|c|c|c|c|c|c|c|c|c|c|c|}
\hline $\begin{array}{l}\text { HMO001S } \\
\text { HM0002S } \\
\text { HM0003S } \\
\text { HMO004S } \\
\text { HM0005S } \\
\text { HM0006S } \\
\text { HM0007S } \\
\text { HMO008S } \\
\text { HMO009S } \\
\text { HM0010S }\end{array}$ & $\begin{array}{l}N \\
N \\
N \\
N \\
N \\
N \\
N \\
N \\
N \\
N\end{array}$ & $\begin{array}{r}N \\
N \\
N \\
N \\
N \\
N \\
N \\
N \\
N\end{array}$ & $\begin{array}{l}N \\
N \\
N \\
N \\
N \\
N \\
N \\
N \\
N \\
N\end{array}$ & $\begin{array}{r}30 \\
20 \\
30 \\
100 \\
50 \\
30 \\
50 \\
30 \\
70 \\
70\end{array}$ & $\begin{array}{r}N \\
N \\
N \\
N \\
N \\
N \\
N \\
N \\
N \\
<20\end{array}$ & $\begin{array}{r}<10 \\
N \\
10 \\
15 \\
<10 \\
<10 \\
N \\
N \\
10 \\
15\end{array}$ & $\begin{array}{l}N \\
N \\
N \\
N \\
N \\
N \\
N \\
N \\
N \\
N\end{array}$ & $\begin{array}{r}70 \\
20 \\
30 \\
150 \\
50 \\
150 \\
50 \\
100 \\
150 \\
70\end{array}$ & $\begin{array}{l}1 \\
1 \\
1 \\
1.2 \\
.3 \\
.1 \\
.1 \\
.2 \\
.3 \\
1.4\end{array}$ & $\begin{array}{l}.2 \\
.1 \\
N \\
.05 \\
N \\
N \\
N \\
.05 \\
.05 \\
N\end{array}$ \\
\hline $\begin{array}{l}\text { HM0011S } \\
\text { HMO012S } \\
\text { HM0013S } \\
\text { HM0014S } \\
\text { HM0015S } \\
\text { HM0016S } \\
\text { HM0017S } \\
\text { HM0018S } \\
\text { HM0019S } \\
\text { HM0020S }\end{array}$ & $\begin{array}{l}N \\
N \\
N \\
N \\
N \\
N \\
N \\
N \\
N \\
N\end{array}$ & $\begin{array}{r}N \\
N \\
N \\
N \\
N \\
N \\
N \\
N \\
N \\
N \\
N\end{array}$ & $\begin{array}{l}N \\
N \\
N \\
N \\
N \\
N \\
N \\
N \\
N \\
N\end{array}$ & $\begin{array}{r}50 \\
30 \\
100 \\
30 \\
50 \\
30 \\
30 \\
70 \\
30 \\
50\end{array}$ & $\begin{array}{r}N \\
N \\
N \\
N \\
N \\
N \\
N \\
<20 \\
N \\
N\end{array}$ & $\begin{array}{r}15 \\
<10 \\
N \\
<10 \\
10 \\
10 \\
10 \\
<10 \\
<10 \\
15\end{array}$ & $\begin{array}{l}N \\
N \\
N \\
N \\
N \\
N \\
N \\
N \\
N \\
N\end{array}$ & $\begin{array}{r}150 \\
50 \\
30 \\
30 \\
100 \\
70 \\
70 \\
30 \\
50 \\
100\end{array}$ & $\begin{array}{r}.3 \\
.7 \\
1.1 \\
.7 \\
.4 \\
1 \\
2 \\
.6 \\
.8 \\
.5\end{array}$ & $\begin{array}{l}N \\
.2 \\
N \\
.1 \\
.1 \\
.45 \\
N \\
N \\
.1\end{array}$ \\
\hline $\begin{array}{l}\text { HM0021S } \\
\text { HM0022S } \\
\text { HM0023S } \\
\text { HM0024S } \\
\text { HM0025S } \\
\text { HM0026S } \\
\text { HM0027S } \\
\text { HM0028S } \\
\text { HM0029S } \\
\text { HM0030S }\end{array}$ & $\begin{array}{l}N \\
N \\
N \\
N \\
N \\
N \\
N \\
N \\
N \\
N\end{array}$ & $\begin{array}{r}<100 \\
N \\
N \\
N \\
N \\
N 100 \\
N \\
N \\
N \\
N\end{array}$ & $\begin{array}{l}\mathbf{N} \\
\mathbf{N} \\
\mathbf{N} \\
\mathbf{N} \\
\mathbf{N} \\
\mathbf{N} \\
\mathbf{N} \\
\mathbf{N} \\
\mathbf{N} \\
\mathbf{N}\end{array}$ & $\begin{array}{l}50 \\
50 \\
50 \\
20 \\
50 \\
70 \\
30 \\
30 \\
70 \\
50\end{array}$ & $\begin{array}{l}N \\
N \\
N \\
N \\
N \\
N \\
N \\
N \\
N \\
N\end{array}$ & $\begin{array}{r}N \\
15 \\
10 \\
<10 \\
<10 \\
15 \\
N \\
10 \\
15 \\
<10\end{array}$ & $\begin{array}{l}N \\
N \\
N \\
N \\
N \\
N \\
N \\
N \\
N \\
N\end{array}$ & $\begin{array}{r}30 \\
150 \\
70 \\
50 \\
50 \\
100 \\
150 \\
200 \\
70 \\
150\end{array}$ & $\begin{array}{l}1 \\
1.3 \\
2.3 \\
.4 \\
.1 \\
.3 \\
.1 \\
.1 \\
.4 \\
<.1\end{array}$ & $\begin{array}{l}N \\
N \\
\dot{N}^{N} \\
N \\
\dot{N}^{1} \\
N \\
N \\
.05\end{array}$ \\
\hline $\begin{array}{l}\text { HMO031S } \\
\text { HMO032S } \\
\text { HMO033S } \\
\text { HMO034S } \\
\text { HMO035S } \\
\text { HMO036S } \\
\text { HMO037S } \\
\text { HMO038S } \\
\text { HMO039S } \\
\text { HMO040S }\end{array}$ & $\begin{array}{l}N \\
N \\
N \\
N \\
N \\
N \\
N \\
N \\
N \\
N\end{array}$ & $\begin{array}{r}N \\
N \\
N \\
N \\
N \\
N \\
N \\
N \\
N \\
N 100\end{array}$ & $\begin{array}{l}N \\
N \\
N \\
N \\
N \\
N \\
N \\
N \\
N \\
N\end{array}$ & $\begin{array}{r}50 \\
50 \\
30 \\
30 \\
30 \\
50 \\
100 \\
50 \\
70 \\
50\end{array}$ & $\begin{array}{l}N \\
N \\
N \\
N \\
N \\
N \\
N \\
N \\
N \\
N\end{array}$ & $\begin{array}{r}N \\
<10 \\
N \\
<10 \\
N \\
N \\
N \\
<10 \\
N \\
10\end{array}$ & $\begin{array}{l}N \\
N \\
N \\
N \\
N \\
N \\
N \\
N \\
N \\
N\end{array}$ & $\begin{array}{r}100 \\
100 \\
50 \\
150 \\
20 \\
50 \\
70 \\
30 \\
70 \\
100\end{array}$ & $\begin{array}{l}.2 \\
.2 \\
.2 \\
.2 \\
.2 \\
.2 \\
.2 \\
.4 \\
.1 \\
.1\end{array}$ & $\begin{array}{l}N \\
N \\
.05 \\
N \\
.1 \\
N \\
.2 \\
.05 \\
N\end{array}$ \\
\hline $\begin{array}{l}\text { HM0041S } \\
\text { HM0042S } \\
\text { HM0043S } \\
\text { HM0044S } \\
\text { HM0045S } \\
\text { HM0046S } \\
\text { HM0047S } \\
\text { HM0048S } \\
\text { HM0049S } \\
\text { HM0050S }\end{array}$ & $\begin{array}{l}N \\
N \\
N \\
N \\
N \\
N \\
N \\
N \\
N \\
N\end{array}$ & $\begin{array}{r}N \\
<100 \\
N \\
<100 \\
N \\
N \\
N \\
N \\
N \\
N\end{array}$ & $\begin{array}{l}N \\
N \\
N \\
N \\
N \\
N \\
N \\
N \\
N \\
N\end{array}$ & $\begin{array}{r}50 \\
30 \\
70 \\
100 \\
70 \\
70 \\
50 \\
50 \\
70 \\
50\end{array}$ & $\begin{array}{r}N \\
N \\
N \\
H \\
<20 \\
N \\
N \\
N \\
N \\
N\end{array}$ & $\begin{array}{r}N \\
<10 \\
<10 \\
10 \\
N \\
N \\
<10 \\
N \\
N \\
N\end{array}$ & $\begin{array}{l}N \\
N \\
N \\
N \\
N \\
N \\
N \\
N \\
N \\
N\end{array}$ & $\begin{array}{r}100 \\
50 \\
70 \\
100 \\
50 \\
50 \\
100 \\
50 \\
30 \\
70\end{array}$ & $\begin{array}{l}.1 \\
.1 \\
.3 \\
.3 \\
.2 \\
.3 \\
.2 \\
.5 \\
.3 \\
.3\end{array}$ & $\begin{array}{l}N \\
N \\
N \\
N \\
N \\
N \\
.3 \\
N \\
N\end{array}$ \\
\hline $\begin{array}{l}\text { HM0100S } \\
\text { HM0101S } \\
\text { HM0102S } \\
\text { HM0103S } \\
\text { HM0104S } \\
\text { HM0105S } \\
\text { HM0106S } \\
\text { HM01075 } \\
\text { HM0108S } \\
\text { HMO109S }\end{array}$ & $\begin{array}{l}N \\
N \\
N \\
N \\
N \\
N \\
N \\
N \\
N \\
N\end{array}$ & $\begin{array}{l}N \\
N \\
N \\
N \\
N \\
N \\
N \\
N \\
N \\
N\end{array}$ & $\begin{array}{l}N \\
N \\
N \\
N \\
N \\
N \\
N \\
N \\
N \\
N\end{array}$ & $\begin{array}{r}50 \\
20 \\
15 \\
20 \\
150 \\
50 \\
50 \\
50 \\
70 \\
50\end{array}$ & $\begin{array}{l}N \\
N \\
N \\
N \\
N \\
N \\
N \\
N \\
N \\
N\end{array}$ & $\begin{array}{r}10 \\
<10 \\
N \\
<10 \\
10 \\
N \\
N \\
N \\
<10 \\
15\end{array}$ & $\begin{array}{l}N \\
N \\
N \\
N \\
N \\
N \\
N \\
N \\
N \\
N\end{array}$ & $\begin{array}{r}70 \\
50 \\
15 \\
150 \\
150 \\
100 \\
100 \\
70 \\
70 \\
70\end{array}$ & $\begin{array}{r}1.1 \\
1.2 \\
.5 \\
.2 \\
.3 \\
.2 \\
.2 \\
.6 \\
.3 \\
2.1\end{array}$ & $\begin{array}{l}.15 \\
N \\
.15 \\
N \\
N \\
N \\
N \\
N \\
N \\
N\end{array}$ \\
\hline $\begin{array}{l}\text { HMO110S } \\
\text { HMO111S } \\
\text { HMO112S } \\
\text { HMO113S } \\
\text { HMO114S }\end{array}$ & $\begin{array}{l}\mathbf{N} \\
\mathbf{N} \\
\mathbf{N} \\
\mathbf{N} \\
\mathbf{N}\end{array}$ & $\begin{array}{r}N \\
N \\
<100 \\
N \\
N\end{array}$ & $\begin{array}{l}N \\
N \\
N \\
N \\
N\end{array}$ & $\begin{array}{r}100 \\
30 \\
50 \\
30 \\
50\end{array}$ & $\begin{array}{l}N \\
N \\
N \\
N \\
N\end{array}$ & $\begin{array}{r}N \\
15 \\
15 \\
N \\
<10\end{array}$ & $\begin{array}{l}N \\
N \\
N \\
N \\
N\end{array}$ & $\begin{array}{r}70 \\
70 \\
100 \\
20 \\
50\end{array}$ & $\begin{array}{l}1 \\
1.1 \\
1 \\
.1 \\
.6\end{array}$ & $\begin{array}{l}N \\
N \\
.2 \\
N \\
N\end{array}$ \\
\hline
\end{tabular}


Table 3. Analytical data for stream-sediment samples collected from the Horn Mountains area--Continued.

Sample Tl ppm-AAS AU ppm-AAS Hg ppm-AAS W ppm-VS F \%-SIE Pt ppb-ICP Pd ppb-ICP Rh ppb-ICP RU ppb-ICP Ir ppb-ICP

\begin{tabular}{|c|c|c|c|c|c|c|c|c|c|c|}
\hline $\begin{array}{l}\text { HMO001S } \\
\text { HM0002S } \\
\text { HM0003S } \\
\text { HM0004S } \\
\text { HM0005S } \\
\text { HMO006S } \\
\text { HM0007S } \\
\text { HMO008S } \\
\text { HM0009S } \\
\text { HM0010S }\end{array}$ & $\begin{array}{l}.3 \\
.35 \\
.25 \\
.6 \\
.3 \\
.4 \\
.3 \\
.35 \\
.4 \\
.2\end{array}$ & $\begin{array}{l}.01 \\
.7 \\
.004 \\
.002 \\
N \\
N \\
.05 \\
.004 \\
.01\end{array}$ & $\begin{array}{l}.12 \\
.1 \\
.18 \\
.19 \\
.07 \\
.81 \\
.41 \\
.05 \\
.06 \\
.22\end{array}$ & $\begin{array}{l}2 \\
6 \\
2 \\
3 \\
1 \\
2 \\
2 \\
1 \\
2 \\
2\end{array}$ & $\begin{array}{l}.06 \\
.06 \\
.12 \\
.09 \\
.15 \\
.04 \\
.06 \\
.05 \\
.04 \\
.05\end{array}$ & $\begin{array}{l}<.5 \\
1 \\
<.5 \\
<1 \\
<.5 \\
<1 \\
<.5 \\
<.5 \\
<1 \\
<.5\end{array}$ & $\begin{array}{l}1.1 \\
.5 \\
.5 \\
1.5 \\
<.5 \\
<.5 \\
<.5 \\
<.5 \\
.6 \\
<.5\end{array}$ & $\begin{array}{l}<.5 \\
<.5 \\
<.5 \\
<.5 \\
<.5 \\
<.5 \\
<.5 \\
<.5 \\
<.5 \\
<.5\end{array}$ & $\begin{array}{l}<.5 \\
<.5 \\
<.5 \\
<.5 \\
<.5 \\
<.5 \\
<.5 \\
<.5 \\
<.5 \\
<.5\end{array}$ & $\begin{array}{l}<.5 \\
<.5 \\
<.5 \\
<.5 \\
<.5 \\
<.5 \\
<.5 \\
<.5 \\
<.5 \\
<.5\end{array}$ \\
\hline $\begin{array}{l}\text { HM0011S } \\
\text { HM0012S } \\
\text { HM0013S } \\
\text { HM0014S } \\
\text { HM0015S } \\
\text { HM0016S } \\
\text { HM0017S } \\
\text { HM0018S } \\
\text { HM0019S } \\
\text { HM0020S }\end{array}$ & $\begin{array}{l}.3 \\
.2 \\
.25 \\
.2 \\
.35 \\
.5 \\
.5 \\
.3 \\
.5 \\
.25\end{array}$ & $\begin{array}{l}N \\
N \\
N \\
N \\
N \\
<.002 \\
<.002 \\
<.002 \\
N \\
N\end{array}$ & $\begin{array}{l}.04 \\
.04 \\
.08 \\
.32 \\
.08 \\
.46 \\
2 \\
.08 \\
.04 \\
.12\end{array}$ & $\begin{array}{l}1 \\
2 \\
3 \\
1 \\
3 \\
4 \\
2 \\
1 \\
5 \\
2\end{array}$ & $\begin{array}{l}.06 \\
.05 \\
.08 \\
.06 \\
.1 \\
.07 \\
.08 \\
.06 \\
.06 \\
.06\end{array}$ & $\begin{array}{l}<.5 \\
<.5 \\
<.5 \\
<.5 \\
<1 \\
<1 \\
<1 \\
<.5 \\
<1 \\
<.5\end{array}$ & $\begin{array}{l}<.5 \\
<.5 \\
.7 \\
<.5 \\
<.5 \\
.5 \\
.7 \\
<.5 \\
<.5 \\
<.5\end{array}$ & $\begin{array}{l}<.5 \\
<.5 \\
<.5 \\
<.5 \\
<.5 \\
<.5 \\
<.5 \\
<.5 \\
<.5 \\
<.5\end{array}$ & $\begin{array}{l}<.5 \\
<.5 \\
1.3 \\
<.5 \\
<.5 \\
<.5 \\
<.5 \\
<.5 \\
<.5 \\
<.5\end{array}$ & $\begin{array}{l}<.5 \\
<.5 \\
<.5 \\
<.5 \\
<.5 \\
<.5 \\
<.5 \\
<.5 \\
<.5 \\
<.5\end{array}$ \\
\hline $\begin{array}{l}\text { HM0021S } \\
\text { HM0022S } \\
\text { HM0023S } \\
\text { HM0024S } \\
\text { HM0025S } \\
\text { HM0026S } \\
\text { HM0027S } \\
\text { HM0028S } \\
\text { HM0029S } \\
\text { HM0030S }\end{array}$ & $\begin{array}{l}.15 \\
.5 \\
.2 \\
.3 \\
.2 \\
.3 \\
.35 \\
.3 \\
.2 \\
.35\end{array}$ & $\begin{array}{l}N \\
N \\
N \\
N \\
N \\
N \\
N \\
N \\
N \\
N\end{array}$ & $\begin{array}{l}1.73 \\
.14 \\
.85 \\
.33 \\
.02 \\
.21 \\
.12 \\
.02 \\
.06 \\
.04\end{array}$ & $\begin{array}{l}2 \\
2 \\
2 \\
5 \\
1 \\
1 \\
N \\
5 \\
2 \\
3\end{array}$ & $\begin{array}{l}.05 \\
.06 \\
.07 \\
.05 \\
.04 \\
.06 \\
.03 \\
.06 \\
.05 \\
.04\end{array}$ & $\begin{array}{l}<.5 \\
<1 \\
<1 \\
<1 \\
<2 \\
<.5 \\
<.5 \\
<.5 \\
<.5 \\
<1\end{array}$ & $\begin{array}{l}<.5 \\
<.5 \\
<1 \\
<.5 \\
<.5 \\
<.5 \\
<.5 \\
<.5 \\
.5 \\
<.5\end{array}$ & $\begin{array}{l}<.5 \\
<.5 \\
<1 \\
<.5 \\
<.5 \\
<.5 \\
<.5 \\
<.5 \\
<.5 \\
<.5\end{array}$ & $\begin{array}{l}<.5 \\
<.5 \\
<1 \\
<.5 \\
<1 \\
<.5 \\
<.5 \\
<.5 \\
<.5 \\
.5\end{array}$ & $\begin{array}{l}<.5 \\
<.5 \\
<1 \\
<.5 \\
<.5 \\
<.5 \\
<.5 \\
<.5 \\
<.5 \\
<.5\end{array}$ \\
\hline $\begin{array}{l}\text { HM0031S } \\
\text { HMO032S } \\
\text { HM0033S } \\
\text { HM0034S } \\
\text { HM0035S } \\
\text { HM0036S } \\
\text { HM0037S } \\
\text { HM0038S } \\
\text { HM0039S } \\
\text { HM0040S }\end{array}$ & $\begin{array}{l}.25 \\
.25 \\
.3 \\
.4 \\
.2 \\
.3 \\
.2 \\
.45 \\
.15 \\
.35\end{array}$ & $\begin{array}{l}.012 \\
N \\
N \\
N \\
N \\
N \\
N \\
<.002 \\
.004 \\
. N\end{array}$ & $\begin{array}{l}.04 \\
3.4 \\
.06 \\
.07 \\
.07 \\
.03 \\
.14 \\
.11 \\
.04 \\
.04\end{array}$ & $\begin{array}{l}2 \\
2 \\
1 \\
1 \\
2 \\
2 \\
2 \\
2 \\
1 \\
1\end{array}$ & $\begin{array}{l}.05 \\
.05 \\
.03 \\
.04 \\
.04 \\
.04 \\
.06 \\
.05 \\
.04 \\
.04\end{array}$ & $\begin{array}{l}<.5 \\
<.5 \\
<.5 \\
<1 \\
<.5 \\
<1 \\
<.5 \\
<1 \\
<.5\end{array}$ & $\begin{array}{l}<.5 \\
<.5 \\
<.5 \\
<.5 \\
<.5 \\
<.5 \\
<.5 \\
<.5 \\
.5 \\
<.5 \\
.5\end{array}$ & $\begin{array}{l}<.5 \\
<.5 \\
<.5 \\
<.5 \\
<.5 \\
<.5 \\
<.5 \\
<.5 \\
<.5 \\
<.5\end{array}$ & $\begin{array}{l}<.5 \\
<.5 \\
<.5 \\
<.5 \\
<.5 \\
<.5 \\
<.5 \\
<.5 \\
.5 \\
<.5 \\
.6\end{array}$ & $\begin{array}{l}<.5 \\
<.5 \\
<.5 \\
<.5 \\
<.5 \\
<.5 \\
<.5 \\
<.5 \\
<.5 \\
<.5\end{array}$ \\
\hline $\begin{array}{l}\text { HM0041S } \\
\text { HM0042S } \\
\text { HM0043S } \\
\text { HM0044S } \\
\text { HM0045S } \\
\text { HM0046S } \\
\text { HM0047S } \\
\text { HM0048S } \\
\text { HM0049S } \\
\text { HM0050S }\end{array}$ & $\begin{array}{l}.2 \\
.35 \\
.4 \\
.4 \\
.15 \\
.2 \\
.25 \\
.25 \\
.2 \\
.2\end{array}$ & $\begin{array}{l}.002 \\
N \\
N \\
N \\
N \\
N \\
<.002 \\
N \\
N \\
<.002\end{array}$ & $\begin{array}{l}.09 \\
.22 \\
.16 \\
.11 \\
.04 \\
1 \\
2.2 \\
.08 \\
.74 \\
5.6\end{array}$ & $\begin{array}{l}2 \\
1 \\
2 \\
3 \\
2 \\
1 \\
1 \\
1 \\
1 \\
2\end{array}$ & $\begin{array}{l}.05 \\
.04 \\
.06 \\
.04 \\
.04 \\
.05 \\
.05 \\
.06 \\
.06 \\
.04\end{array}$ & $\begin{array}{l}<.5 \\
<1 \\
<1 \\
<1 \\
<.5 \\
<.5 \\
<.5 \\
<.5 \\
<.5 \\
<.5\end{array}$ & $\begin{array}{l}<.5 \\
<.5 \\
.5 \\
<.5 \\
<.5 \\
<.5 \\
<.5 \\
.7 \\
<.5 \\
<.5\end{array}$ & $\begin{array}{l}<.5 \\
<.5 \\
<.5 \\
<.5 \\
<.5 \\
<.5 \\
<.5 \\
<.5 \\
<.5 \\
<.5\end{array}$ & $\begin{array}{l}<.5 \\
.7 \\
<.5 \\
<.5 \\
<.5 \\
<.5 \\
<.5 \\
<.5 \\
<.5 \\
<.5\end{array}$ & $\begin{array}{l}<.5 \\
<.5 \\
<.5 \\
<.5 \\
<.5 \\
<.5 \\
<.5 \\
<.5 \\
<.5 \\
<.5\end{array}$ \\
\hline $\begin{array}{l}\text { HM0100S } \\
\text { HM0101S } \\
\text { HM0102S } \\
\text { HM0103S } \\
\text { HM0104S } \\
\text { HM0105S } \\
\text { HM0106S } \\
\text { HM0107S } \\
\text { HM0108S } \\
\text { HM0109S }\end{array}$ & $\begin{array}{l}.3 \\
.35 \\
.3 \\
.5 \\
.2 \\
.45 \\
.2 \\
.15 \\
.15 \\
.25\end{array}$ & $\begin{array}{l}.008 \\
.25 \\
.15 \\
.002 \\
N \\
.006 \\
\dot{N}_{N} \\
.018 \\
\dot{N}^{0}\end{array}$ & $\begin{array}{l}.16 \\
.16 \\
.07 \\
.07 \\
.41 \\
.18 \\
.04 \\
.07 \\
.03 \\
.1\end{array}$ & $\begin{array}{r}4 \\
10 \\
6 \\
3 \\
2 \\
2 \\
2 \\
N \\
2 \\
2\end{array}$ & $\begin{array}{l}.06 \\
.06 \\
.06 \\
.04 \\
.06 \\
.05 \\
.05 \\
.04 \\
.05 \\
.05\end{array}$ & $\begin{array}{l}<.5 \\
<.5 \\
<1 \\
<1 \\
<.5 \\
2 \\
<.5 \\
<.5 \\
<.5 \\
<.5\end{array}$ & $\begin{array}{r}.7 \\
<1 \\
<.9 \\
<.5 \\
.5 \\
2.5 \\
<.5 \\
<.5 \\
<.5 \\
1.3\end{array}$ & $\begin{array}{l}<.5 \\
<.5 \\
<1 \\
<.5 \\
<.5 \\
<.5 \\
<.5 \\
<.5 \\
<.5 \\
<.5\end{array}$ & $\begin{array}{l}<.5 \\
<.5 \\
<1 \\
<.5 \\
<.5 \\
1.5 \\
<.5 \\
<.5 \\
<.5 \\
<.5\end{array}$ & $\begin{array}{l}<.5 \\
<.5 \\
<1 \\
<.5 \\
<.5 \\
<.5 \\
<.5 \\
<.5 \\
<.5 \\
<.5\end{array}$ \\
\hline $\begin{array}{l}\text { HMO110S } \\
\text { HM0111S } \\
\text { HM0112S } \\
\text { HM0113S } \\
\text { HMO114S }\end{array}$ & $\begin{array}{l}.3 \\
.25 \\
.45 \\
.2 \\
.5\end{array}$ & $\begin{array}{l}N \\
N \\
.026 \\
N \\
N\end{array}$ & $\begin{array}{l}.1 \\
.25 \\
.35 \\
.05 \\
.08\end{array}$ & $\begin{array}{l}2 \\
3 \\
2 \\
2 \\
7\end{array}$ & $\begin{array}{l}.06 \\
.05 \\
.06 \\
.13 \\
.04\end{array}$ & $\begin{array}{l}<.5 \\
<.5 \\
<1 \\
<.5\end{array}$ & $\begin{array}{l}.7 \\
<.5 \\
1.3 \\
<.5 \\
<.5\end{array}$ & $\begin{array}{l}<.5 \\
<.5 \\
<.5 \\
<.5 \\
<.5\end{array}$ & $\begin{array}{l}<.5 \\
<.5 \\
.5 \\
<.5 \\
<.5\end{array}$ & $\begin{array}{l}<.5 \\
<.5 \\
<.5 \\
<.5 \\
<.5\end{array}$ \\
\hline
\end{tabular}


Table 3. Analytical data for stream-sediment samples collected from the Horn Mountains area--Continued.

\begin{tabular}{|c|c|c|c|c|c|c|c|c|c|c|}
\hline Sample & Lat i tude & Longi tude & Ag ppm-ICP & As ppm-ICP & Au ppm-ICP & Bi ppm-ICP & Cd ppm-ICP & Cu ppm-ICP & Ho ppm-ICP & Pb Ppm-ICP \\
\hline $\begin{array}{l}\text { HMO115S } \\
\text { HMO116S } \\
\text { HM0117S } \\
\text { HM0118S } \\
\text { HMO119S } \\
\text { HM0120S } \\
\text { HM0121S } \\
\text { HM0122S } \\
\text { HMO123S } \\
\text { HM0124S }\end{array}$ & $\begin{array}{rrr}61 & 43 & 48 \\
61 & 43 & 44 \\
61 & 45 & 54 \\
61 & 47 & 19 \\
61 & 49 & 2 \\
61 & 49 & 11 \\
61 & 49 & 10 \\
61 & 51 & 8 \\
61 & 51 & 53 \\
61 & 51 & 1\end{array}$ & $\begin{array}{rrr}158 & 33 & 56 \\
158 & 34 & 2 \\
158 & 34 & 38 \\
158 & 32 & 6 \\
158 & 28 & 9 \\
158 & 23 & 47 \\
158 & 23 & 42 \\
158 & 19 & 16 \\
158 & 26 & 53 \\
158 & 28 & 25\end{array}$ & $\begin{array}{l}.13 \\
N \\
N \\
.1 \\
.25 \\
N \\
N \\
N \\
.095\end{array}$ & $\begin{array}{c}28 \\
57 \\
13 \\
120 \\
130 \\
15 \\
14 \\
6.6 \\
53 \\
4\end{array}$ & $\begin{array}{l}N \\
N \\
N \\
N \\
N \\
N \\
N \\
N \\
N \\
N\end{array}$ & $\begin{array}{l}N \\
N \\
N \\
N \\
N \\
N \\
N \\
N \\
.74 \\
N\end{array}$ & $\begin{array}{l}.25 \\
.24 \\
.3 \\
.53 \\
.7 \\
.13 \\
.18 \\
.11 \\
.36 \\
.097\end{array}$ & $\begin{array}{l}25 \\
19 \\
9.8 \\
24 \\
12 \\
7.1 \\
9.2 \\
8.7 \\
10 \\
7.4\end{array}$ & $\begin{array}{l}1 \\
1.1 \\
1.2 \\
.7 \\
.9 \\
.46 \\
.56 \\
.39 \\
.75 \\
.53\end{array}$ & $\begin{array}{c}15 \\
12 \\
11 \\
17 \\
25 \\
8.5 \\
9.5 \\
7.1 \\
17 \\
6.2\end{array}$ \\
\hline $\begin{array}{l}\text { HMO125S } \\
\text { HMO126S } \\
\text { HMO127S } \\
\text { HM0128S } \\
\text { HM0129S } \\
\text { HM0130S } \\
\text { HM0131S } \\
\text { HM0132S } \\
\text { HM0133S } \\
\text { HM0134S }\end{array}$ & $\begin{array}{rrr}61 & 51 & 0 \\
61 & 54 & 57 \\
61 & 53 & 53 \\
61 & 54 & 56 \\
61 & 51 & 14 \\
61 & 51 & 21 \\
61 & 47 & 59 \\
61 & 45 & 51 \\
61 & 55 & 38 \\
61 & 58 & 11\end{array}$ & $\begin{array}{rrr}158 & 28 & 17 \\
158 & 19 & 1 \\
158 & 17 & 24 \\
158 & 13 & 9 \\
158 & 14 & 9 \\
158 & 9 & 9 \\
158 & 15 & 19 \\
158 & 15 & 50 \\
158 & 45 & 25 \\
158 & 55 & 19\end{array}$ & $\begin{array}{l}N \\
N \\
\dot{N} \\
N \\
N \\
N \\
N \\
N \\
N\end{array}$ & $\begin{array}{l}29 \\
10 \\
5.2 \\
6.3 \\
4.5 \\
6.2 \\
19 \\
11 \\
5.4 \\
6.2\end{array}$ & $\begin{array}{l}N \\
N \\
N \\
N \\
N \\
N \\
N \\
N \\
N \\
N\end{array}$ & $\begin{array}{l}N \\
N \\
N \\
N \\
N \\
N \\
N \\
N \\
N \\
N\end{array}$ & $\begin{array}{l}.18 \\
.15 \\
.28 \\
.14 \\
.15 \\
.11 \\
.12 \\
.14 \\
.18 \\
.16\end{array}$ & $\begin{array}{l}12 \\
12 \\
15 \\
13 \\
10 \\
6.6 \\
13 \\
13 \\
16 \\
11\end{array}$ & $\begin{array}{l}.57 \\
.46 \\
.57 \\
.73 \\
.37 \\
.21 \\
.57 \\
.72 \\
.59 \\
.56\end{array}$ & $\begin{array}{l}10 \\
7.5 \\
9.8 \\
8.4 \\
7 \\
4.6 \\
7 \\
6.5 \\
10 \\
7.4\end{array}$ \\
\hline $\begin{array}{l}\text { HMO135S } \\
\text { HMO136S } \\
\text { HM0137S } \\
\text { HM0138S } \\
\text { HM0139S } \\
\text { HMO140S } \\
\text { HM0141S } \\
\text { HM0142S } \\
\text { HM0143S } \\
\text { HM0144S }\end{array}$ & $\begin{array}{rrr}61 & 58 & 8 \\
61 & 59 & 56 \\
61 & 58 & 20 \\
61 & 54 & 30 \\
61 & 59 & 13 \\
61 & 51 & 14 \\
61 & 52 & 47 \\
61 & 52 & 17 \\
61 & 49 & 34 \\
61 & 45 & 56\end{array}$ & $\begin{array}{rrr}158 & 49 & 25 \\
158 & 43 & 12 \\
158 & 39 & 55 \\
158 & 33 & 39 \\
158 & 21 & 18 \\
158 & 34 & 2 \\
158 & 41 & 31 \\
158 & 53 & 23 \\
158 & 48 & 0 \\
158 & 49 & 3\end{array}$ & $\begin{array}{l}.07 \\
N \\
N \\
N \\
N \\
.087 \\
i^{-23} \\
. \\
N\end{array}$ & $\begin{array}{l}3^{N} \\
11 \\
3.5 \\
N \\
6.8 \\
18 \\
67 \\
8.9 \\
3.8\end{array}$ & $\begin{array}{l}N \\
N \\
N \\
N \\
N \\
N \\
N \\
N \\
N \\
N\end{array}$ & $\begin{array}{l}N \\
N \\
N \\
N \\
N \\
N \\
N \\
N \\
N \\
N\end{array}$ & $\begin{array}{l}.17 \\
.13 \\
.17 \\
.33 \\
.099 \\
.22 \\
.23 \\
.15 \\
.098 \\
.12\end{array}$ & $\begin{array}{l}12 \\
14 \\
20 \\
19 \\
13 \\
12 \\
19 \\
59 \\
17 \\
11\end{array}$ & $\begin{array}{l}.6 \\
.52 \\
.96 \\
.63 \\
.65 \\
.47 \\
1 \\
.43 \\
.29 \\
.56\end{array}$ & $\begin{array}{c}7.7 \\
6.7 \\
10 \\
11 \\
7.5 \\
6.9 \\
13 \\
11 \\
6.9 \\
5.8\end{array}$ \\
\hline $\begin{array}{l}\text { HM0145S } \\
\text { HMO146S } \\
\text { HMO147S } \\
\text { HM0148S } \\
\text { HM0200S } \\
\text { HM0201S } \\
\text { HM0202S } \\
\text { HM0203S } \\
\text { HM0204S } \\
\text { HM0205S }\end{array}$ & $\begin{array}{lll}61 & 49 & 55 \\
61 & 43 & 11 \\
61 & 39 & 43 \\
61 & 43 & 44 \\
61 & 38 & 25 \\
61 & 38 & 59 \\
61 & 41 & 15 \\
61 & 42 & 54 \\
61 & 44 & 36 \\
61 & 46 & 9\end{array}$ & $\begin{array}{lll}158 & 57 & 10 \\
158 & 53 & 35 \\
158 & 50 & 51 \\
158 & 48 & 57 \\
158 & 24 & 46 \\
158 & 21 & 47 \\
158 & 21 & 18 \\
158 & 16 & 33 \\
158 & 24 & 20 \\
158 & 25 & 54\end{array}$ & $\begin{array}{l}N \\
.23 \\
N \\
N \\
N \\
N \\
.083 \\
N \\
.11 \\
.53\end{array}$ & $\begin{array}{c}5.1 \\
72 \\
12 \\
1.4 \\
14 \\
10 \\
1.9 \\
4.8 \\
34 \\
250\end{array}$ & $\begin{array}{l}N \\
N \\
N \\
N \\
N \\
N \\
N \\
N \\
N \\
N\end{array}$ & $\begin{array}{c}N \\
1.7 \\
N \\
N \\
N \\
N \\
N \\
N \\
6.4\end{array}$ & $\begin{array}{l}.088 \\
.12 \\
.12 \\
.82 \\
.24 \\
.22 \\
.21 \\
.15 \\
.56 \\
.69\end{array}$ & $\begin{array}{l}8.5 \\
65 \\
20 \\
12 \\
13 \\
14 \\
14 \\
12 \\
13 \\
71\end{array}$ & $\begin{array}{l}.33 \\
.63 \\
.71 \\
.55 \\
.78 \\
.7 \\
.35 \\
.46 \\
1.1 \\
.65\end{array}$ & $\begin{array}{c}5.1 \\
9.5 \\
8 \\
6.6 \\
11 \\
8.2 \\
7.8 \\
5.8 \\
14 \\
27\end{array}$ \\
\hline $\begin{array}{l}\text { HM0206S } \\
H M 0207 S \\
H M 0208 S \\
H M 0209 S \\
H M 0210 S \\
H M 0211 S \\
H M 0212 S \\
H M 0213 S \\
H M 0214 S \\
H M 0215 S\end{array}$ & $\begin{array}{rrr}61 & 48 & 27 \\
61 & 41 & 17 \\
61 & 42 & 37 \\
61 & 44 & 38 \\
61 & 46 & 43 \\
61 & 47 & 27 \\
61 & 48 & 29 \\
61 & 48 & 31 \\
61 & 52 & 7 \\
61 & 53 & 22\end{array}$ & $\begin{array}{rrr}158 & 18 & 58 \\
158 & 34 & 4 \\
158 & 34 & 30 \\
158 & 35 & 7 \\
158 & 35 & 11 \\
158 & 29 & 42 \\
158 & 27 & 18 \\
158 & 27 & 23 \\
158 & 25 & 31 \\
158 & 26 & 58\end{array}$ & $\begin{array}{l}N \\
.074 \\
N \\
.11 \\
\hat{H}^{-25} \\
.5 \\
.5 \\
.076 \\
N \\
N\end{array}$ & $\begin{array}{c}85 \\
100 \\
20 \\
14 \\
17 \\
220 \\
180 \\
130 \\
8.5 \\
5.4\end{array}$ & $\begin{array}{l}N \\
N \\
N \\
N \\
N \\
N \\
N \\
N \\
N \\
N\end{array}$ & $\begin{array}{l}N \\
N \\
N \\
N \\
N \\
N \\
4 \\
1.6 \\
N \\
N\end{array}$ & $\begin{array}{l}.24 \\
.27 \\
.31 \\
.25 \\
.22 \\
.62 \\
.68 \\
.46 \\
.099 \\
.13\end{array}$ & $\begin{array}{l}18 \\
14 \\
21 \\
14 \\
11 \\
15 \\
22 \\
16 \\
5.8 \\
6.1\end{array}$ & $\begin{array}{c}1.1 \\
1.3 \\
1.2 \\
.93 \\
.56 \\
1.6 \\
.97 \\
1.1 \\
.41 \\
.51\end{array}$ & $\begin{array}{c}13 \\
11 \\
12 \\
12 \\
9 \\
33 \\
57 \\
14 \\
6.2 \\
6.7\end{array}$ \\
\hline $\begin{array}{l}\text { HMO216S } \\
\text { HMO217S } \\
\text { HMO218S } \\
\text { HMO219S } \\
\text { HMO220S } \\
\text { HMO221S } \\
\text { HMO222S } \\
\text { HMO223S } \\
\text { HMO224S } \\
\text { HMO225S }\end{array}$ & $\begin{array}{rrr}61 & 53 & 55 \\
61 & 53 & 54 \\
61 & 54 & 8 \\
61 & 50 & 31 \\
61 & 51 & 29 \\
61 & 48 & 15 \\
61 & 46 & 49 \\
61 & 54 & 46 \\
61 & 57 & 49 \\
61 & 57 & 20\end{array}$ & $\begin{array}{rrr}158 & 23 & 16 \\
158 & 20 & 54 \\
158 & 15 & 22 \\
158 & 16 & 9 \\
158 & 11 & 7 \\
158 & 12 & 50 \\
158 & 13 & 31 \\
158 & 44 & 13 \\
158 & 57 & 22 \\
158 & 51 & 53\end{array}$ & $\begin{array}{l}N \\
N \\
N \\
N \\
N \\
N \\
N \\
N \\
N \\
N\end{array}$ & $\begin{array}{l}18 \\
5.7 \\
4.3 \\
2.8 \\
5.4 \\
1.8 \\
5.2 \\
4.3 \\
4.1 \\
2.8\end{array}$ & $\begin{array}{l}N \\
N \\
N \\
N \\
N \\
N \\
N \\
N \\
N \\
N\end{array}$ & $\begin{array}{l}N \\
N \\
N \\
N \\
N \\
N \\
N \\
N \\
N \\
N\end{array}$ & $\begin{array}{l}.11 \\
.19 \\
.13 \\
.13 \\
.11 \\
.12 \\
.15 \\
.075 \\
.13 \\
.16\end{array}$ & $\begin{array}{l}9.8 \\
16 \\
12 \\
10 \\
14 \\
7.5 \\
14 \\
13 \\
9.4 \\
7.3\end{array}$ & $\begin{array}{l}.37 \\
.48 \\
.48 \\
.41 \\
.69 \\
.2 \\
.35 \\
.49 \\
.46 \\
.37\end{array}$ & $\begin{array}{l}7.4 \\
9 \\
6.1 \\
5.5 \\
6.4 \\
3.6 \\
5.8 \\
5.9 \\
5.4 \\
4.9\end{array}$ \\
\hline $\begin{array}{l}\text { HM0226S } \\
\text { HM0227S } \\
\text { HM0228S } \\
\text { HM0229S } \\
\text { HM0230S }\end{array}$ & $\begin{array}{lll}61 & 58 & 15 \\
61 & 59 & 53 \\
61 & 58 & 36 \\
61 & 59 & 11 \\
61 & 52 & 31\end{array}$ & $\begin{array}{rrr}158 & 45 & 20 \\
158 & 40 & 1 \\
158 & 33 & 32 \\
158 & 30 & 29 \\
158 & 34 & 28\end{array}$ & $\begin{array}{l}N \\
N \\
\dot{N}^{14} \\
.078\end{array}$ & $\begin{array}{l}1.3 \\
3.5 \\
79 \\
16 \\
6.5\end{array}$ & $\begin{array}{l}N \\
N \\
N \\
N \\
N\end{array}$ & $\begin{array}{l}N \\
N \\
N \\
N \\
N\end{array}$ & $\begin{array}{l}.12 \\
.14 \\
.2 \\
.25 \\
.23\end{array}$ & $\begin{array}{l}15 \\
13 \\
22 \\
15 \\
13\end{array}$ & $\begin{array}{l}.64 \\
.53 \\
.85 \\
.91 \\
.53\end{array}$ & $\begin{array}{l}7.8 \\
7.2 \\
7.8 \\
9.6 \\
8.1\end{array}$ \\
\hline
\end{tabular}


Table 3. Analytical data for stream-sediment samples collected from the Horn Mountains area--Continued.

Sample sb ppm-ICP Zn ppm-ICP Ca $x$-sas fe $x$-sas Mg $x$-sas Na $x$-sas $p$-sas ti $x$-sas Ag ppm-sas As ppm-sas

\begin{tabular}{|c|c|c|c|c|c|c|c|c|c|c|}
\hline $\begin{array}{l}\text { HMO115s } \\
\text { HMO116S } \\
\text { HMO117S } \\
\text { HM0118S } \\
\text { HM0119S } \\
\text { HM0120S } \\
\text { HM0121S } \\
\text { HMO122S } \\
\text { HM0123S } \\
\text { HM0124S }\end{array}$ & $\begin{array}{c}6 \\
1.1 \\
N \\
7.9 \\
45 \\
N \\
1.2 \\
N .1 \\
N\end{array}$ & $\begin{array}{r}86 \\
64 \\
87 \\
95 \\
100 \\
73 \\
62 \\
60 \\
81 \\
50\end{array}$ & $\begin{array}{l}.2 \\
.3 \\
.2 \\
.2 \\
.15 \\
.2 \\
.7 \\
.2 \\
.3 \\
.15\end{array}$ & $\begin{array}{l}3 \\
5 \\
5 \\
3 \\
5 \\
3 \\
5 \\
5 \\
3 \\
2\end{array}$ & $\begin{array}{l}.5 \\
.7 \\
.3 \\
.7 \\
.7 \\
.7 \\
.7 \\
1.7 \\
.2\end{array}$ & $\begin{array}{l}3 \\
3 \\
3 \\
3 \\
3 \\
3 \\
2 \\
2 \\
2 \\
3\end{array}$ & $\begin{array}{l}N \\
N \\
N \\
N \\
N \\
N \\
N \\
N \\
N \\
N\end{array}$ & \begin{tabular}{c}
.7 \\
.7 \\
.7 \\
.5 \\
.7 \\
.7 \\
$>19$ \\
1 \\
\multicolumn{1}{c}{.5} \\
.15
\end{tabular} & $\begin{array}{l}N \\
N \\
N \\
N \\
N \\
N \\
N \\
N \\
N \\
N\end{array}$ & $\begin{array}{l}N \\
N \\
N \\
N \\
N \\
N \\
N \\
N \\
N \\
N\end{array}$ \\
\hline $\begin{array}{l}\text { HMO125S } \\
\text { HM0126S } \\
\text { HM0127S } \\
\text { HM0128S } \\
\text { HM0129S } \\
\text { HM0130S } \\
\text { HM0131S } \\
\text { HM0132S } \\
\text { HM0133S } \\
\text { HM0134S }\end{array}$ & $\begin{array}{l}3 \\
N \\
N \\
.97 \\
N \\
1.2 \\
N \\
.99 \\
.71 \\
.^{2}\end{array}$ & $\begin{array}{l}70 \\
58 \\
64 \\
80 \\
73 \\
53 \\
70 \\
70 \\
75 \\
74\end{array}$ & $\begin{array}{l}.3 \\
.15 \\
.15 \\
.15 \\
.1 \\
.1 \\
.15 \\
.15 \\
.07 \\
<.05\end{array}$ & $\begin{array}{l}3 \\
3 \\
3 \\
7 \\
1.5 \\
3 \\
3 \\
5 \\
2 \\
2\end{array}$ & $\begin{array}{l}1 \\
.2 \\
.2 \\
1 \\
.3 \\
.5 \\
.5 \\
.5 \\
.2 \\
.2\end{array}$ & $\begin{array}{l}1.5 \\
2 \\
2 \\
3 \\
3 \\
2 \\
2 \\
1.5 \\
2.7 \\
.7\end{array}$ & $\begin{array}{l}N \\
N \\
N \\
N \\
N \\
N \\
N \\
N \\
N \\
N \\
N\end{array}$ & $\begin{array}{c}1 \\
.3 \\
.3 \\
.5 \\
.2 \\
.3 \\
.5 \\
1.2 \\
.2 \\
.15\end{array}$ & $\begin{array}{l}N \\
N \\
N \\
N \\
N \\
N \\
N \\
N \\
N \\
N \\
N\end{array}$ & $\begin{array}{l}N \\
N \\
N \\
N \\
N \\
N \\
N \\
N \\
N \\
N \\
N\end{array}$ \\
\hline $\begin{array}{l}\text { HM0135S } \\
\text { HM0136S } \\
\text { HMO137S } \\
\text { HM0138S } \\
\text { HMO139S } \\
\text { HM0140S } \\
\text { HM0141S } \\
\text { HM0142S } \\
\text { HM0143S } \\
\text { HM0144S }\end{array}$ & $\begin{array}{c}.72 \\
N \\
1.8 \\
N \\
N \\
N \\
N \\
17 \\
2.6 \\
N\end{array}$ & $\begin{array}{l}73 \\
76 \\
95 \\
71 \\
73 \\
61 \\
76 \\
77 \\
61 \\
76\end{array}$ & $\begin{array}{l}.07 \\
.15 \\
.15 \\
.1 \\
.05 \\
.1 \\
.1 \\
.1 \\
.07 \\
.1\end{array}$ & $\begin{array}{l}2 \\
5 \\
5 \\
2 \\
3 \\
3 \\
3 \\
2 \\
1.5 \\
3\end{array}$ & $\begin{array}{l}.3 \\
.5 \\
.5 \\
.2 \\
.5 \\
.3 \\
.2 \\
.3 \\
.2 \\
.3\end{array}$ & $\begin{array}{l}1.5 \\
2.5 \\
1.5 \\
2 \\
2 \\
2.2 \\
2 \\
3 \\
2\end{array}$ & $\begin{array}{l}N \\
N \\
N \\
N \\
N \\
N \\
N \\
N \\
N \\
N \\
N\end{array}$ & $\begin{array}{l}.2 \\
.5 \\
.5 \\
.15 \\
.3 \\
.2 \\
.2 \\
.3 \\
.2 \\
.3\end{array}$ & $\begin{array}{l}\mathbf{N} \\
\mathbf{N} \\
\mathbf{N} \\
\mathbf{N} \\
\mathbf{N} \\
\mathbf{N} \\
\mathbf{N} \\
\mathbf{N} \\
\mathbf{N} \\
\mathbf{N}\end{array}$ & $\begin{array}{l}N \\
N \\
N \\
N \\
N \\
N \\
N \\
N \\
N \\
N\end{array}$ \\
\hline $\begin{array}{l}\text { HM0145s } \\
\text { HM0146S } \\
\text { HM0147S } \\
\text { HM0148S } \\
\text { HM0200S } \\
\text { HM0201S } \\
\text { HM0202S } \\
\text { HM0203S } \\
\text { HM0204S } \\
\text { HM0205S }\end{array}$ & $\begin{array}{c}22 \\
1.4 \\
N \\
3.8 \\
1.6 \\
.76 \\
1.4 \\
7.5 \\
24\end{array}$ & $\begin{array}{r}60 \\
77 \\
100 \\
84 \\
80 \\
79 \\
60 \\
71 \\
59 \\
120\end{array}$ & $\begin{array}{l}.1 \\
.07 \\
.05 \\
.1 \\
.1 \\
.1 \\
.15 \\
.1 \\
.15 \\
.2\end{array}$ & $\begin{array}{l}2 \\
3 \\
3 \\
3 \\
5 \\
3 \\
5 \\
3 \\
3 \\
3\end{array}$ & $\begin{array}{l}.3 \\
.3 \\
.3 \\
.3 \\
.5 \\
.3 \\
.3 \\
.3 \\
.5 \\
.5\end{array}$ & $\begin{array}{l}3 \\
2 \\
1.5 \\
2 \\
3 \\
2 \\
2 \\
1.5 \\
2 \\
2\end{array}$ & $\begin{array}{l}N \\
N \\
N \\
N \\
N \\
N \\
N \\
N \\
N \\
N \\
N \\
N\end{array}$ & $\begin{array}{l}.3 \\
.3 \\
.3 \\
.3 \\
.5 \\
.3 \\
.5 \\
.5 \\
.5 \\
.5\end{array}$ & $\begin{array}{l}N \\
N \\
N \\
N \\
N \\
N \\
N \\
N \\
N \\
N \\
<.5\end{array}$ & $\begin{array}{l}N \\
N \\
N \\
N \\
N \\
N \\
N \\
N \\
N \\
N\end{array}$ \\
\hline $\begin{array}{l}\text { HM0206s } \\
\text { HM0207S } \\
\text { HM0208S } \\
\text { HM0209S } \\
\text { HM0210S } \\
\text { HM0211S } \\
\text { HM0212S } \\
\text { HM0213s } \\
\text { HM0214S } \\
\text { HM0215S }\end{array}$ & $\begin{array}{c}2.5 \\
1.5 \\
N \\
1.9 \\
1.6 \\
57 \\
29 \\
7 \\
N \\
1.3\end{array}$ & $\begin{array}{r}83 \\
66 \\
73 \\
69 \\
67 \\
110 \\
120 \\
70 \\
51 \\
51\end{array}$ & $\begin{array}{l}.2 \\
.15 \\
.2 \\
.1 \\
.3 \\
.07 \\
.15 \\
.15 \\
.3 \\
.05\end{array}$ & $\begin{array}{l}5 \\
3 \\
3 \\
3 \\
3 \\
2 \\
3 \\
3 \\
2 \\
1.5\end{array}$ & $\begin{array}{l}1 \\
.3 \\
.3 \\
.3 \\
.7 \\
.3 \\
.7 \\
.7 \\
.5 \\
.15\end{array}$ & $\begin{array}{l}2 \\
3 \\
3 \\
3 \\
3 \\
2 \\
2 \\
1.5 \\
2 \\
3\end{array}$ & $\begin{array}{l}N \\
N \\
N \\
N \\
N \\
N \\
N \\
N \\
N \\
N \\
N\end{array}$ & $\begin{array}{l}1 \\
.3 \\
.5 \\
.5 \\
.5 \\
.2 \\
.2 \\
.5 \\
.7 \\
.2\end{array}$ & $\begin{array}{l}N \\
N \\
N \\
N \\
N \\
N \\
N \\
<.5 \\
N \\
N \\
N\end{array}$ & $\begin{array}{l}N \\
N \\
N \\
N \\
N \\
N \\
N \\
N \\
N \\
N\end{array}$ \\
\hline $\begin{array}{l}\text {HMO216S } \\
\text { HM0217S } \\
\text { HM0218S } \\
\text { HM0219S } \\
\text { HM0220S } \\
\text { HM0221S } \\
\text { HM0222S } \\
\text { HM0223S } \\
\text { HM0224S } \\
\text { HM0225S }\end{array}$ & $\begin{array}{l}1.5 \\
N \\
N \\
.81 \\
N \\
N \\
N \\
N \\
1.3 \\
1.8\end{array}$ & $\begin{array}{l}68 \\
61 \\
62 \\
73 \\
87 \\
48 \\
62 \\
78 \\
56 \\
47\end{array}$ & $\begin{array}{l}.15 \\
.2 \\
.1 \\
.07 \\
.1 \\
.15 \\
.15 \\
.15 \\
.07 \\
.15\end{array}$ & $\begin{array}{l}1.5 \\
2 \\
3 \\
3 \\
3 \\
2 \\
2 \\
3 \\
2 \\
2\end{array}$ & $\begin{array}{l}.5 \\
.3 \\
.3 \\
.5 \\
.5 \\
.5 \\
.2 \\
.5 \\
.15 \\
.3\end{array}$ & $\begin{array}{l}3 \\
1.5 \\
1.5 \\
3 \\
2 \\
2 \\
1.5 \\
2 \\
1 \\
1.5\end{array}$ & $\begin{array}{l}N \\
N \\
N \\
N \\
N \\
N \\
N \\
N \\
N \\
N \\
N\end{array}$ & $\begin{array}{l}.3 \\
.3 \\
.5 \\
.2 \\
.5 \\
.3 \\
.3 \\
.3 \\
.5 \\
.5\end{array}$ & $\begin{array}{l}N \\
N \\
N \\
N \\
N \\
N \\
N \\
N \\
N \\
N\end{array}$ & $\begin{array}{l}N \\
N \\
N \\
N \\
N \\
N \\
N \\
N \\
N \\
N\end{array}$ \\
\hline $\begin{array}{l}\text { HM0226s } \\
\text { HM0227S } \\
\text { HM0228S } \\
\text { HM0229S } \\
\text { HM0230S }\end{array}$ & $\begin{array}{l}N \\
2.1 \\
5.7 \\
2.9 \\
N\end{array}$ & $\begin{array}{l}79 \\
73 \\
79 \\
85 \\
75\end{array}$ & $\begin{array}{l}.07 \\
.1 \\
.07 \\
.05 \\
.2\end{array}$ & $\begin{array}{l}2 \\
3 \\
3 \\
3 \\
5\end{array}$ & $\begin{array}{l}.5 \\
.5 \\
.5 \\
.3 \\
.3\end{array}$ & $\begin{array}{l}1.5 \\
2 \\
2 \\
3 \\
3\end{array}$ & $\begin{array}{l}N \\
N \\
N \\
N \\
N\end{array}$ & $\begin{array}{l}.2 \\
.5 \\
.5 \\
.2 \\
.3\end{array}$ & $\begin{array}{l}N \\
N \\
N \\
N \\
N\end{array}$ & $\begin{array}{l}\mathbf{N} \\
\mathbf{N} \\
\mathbf{N} \\
\mathbf{N} \\
\mathbf{N}\end{array}$ \\
\hline
\end{tabular}


Table 3. Analytical data for stream-sediment samples collected from the Horn Mountains area--Continued.

\begin{tabular}{|c|c|c|c|c|c|c|c|c|c|c|}
\hline Sample & Au ppm-sas & B ppm-sas & Ba ppm-Sas & Be ppm-SOS & Bi ppm-Sas & Cd ppm-SQS & Co ppm-Sas & Cr ppm-SQS & Cu ppm-sas & Ga ppm-SOS \\
\hline $\begin{array}{l}\text { HMO115S } \\
\text { HMO116S } \\
\text { HM0117S } \\
\text { HM0118S } \\
\text { HM0119S } \\
\text { HM0120S } \\
\text { HM0121S } \\
\text { HM0122S } \\
\text { HM0123S } \\
\text { HM0124S }\end{array}$ & $\begin{array}{l}N \\
N \\
N \\
N \\
N \\
N \\
N \\
N \\
N \\
N\end{array}$ & $\begin{array}{r}10 \\
10 \\
<10 \\
20 \\
20 \\
20 \\
15 \\
N \\
150 \\
<10\end{array}$ & $\begin{array}{r}300 \\
700 \\
500 \\
500 \\
300 \\
300 \\
1,500 \\
300 \\
1,000 \\
500\end{array}$ & $\begin{array}{r}<1 \\
<1 \\
N \\
<1 \\
N \\
N \\
N \\
<1 \\
N \\
<1 \\
N\end{array}$ & $\begin{array}{l}N \\
N \\
N \\
N \\
N \\
N \\
N \\
N \\
N \\
N\end{array}$ & $\begin{array}{l}N \\
N \\
N \\
N \\
N \\
N \\
N \\
N \\
N\end{array}$ & $\begin{array}{l}10 \\
15 \\
15 \\
15 \\
15 \\
15 \\
20 \\
30 \\
15 \\
N\end{array}$ & $\begin{array}{r}50 \\
150 \\
100 \\
500 \\
500 \\
500 \\
500 \\
1,000 \\
200 \\
50\end{array}$ & $\begin{array}{r}30 \\
50 \\
30 \\
50 \\
30 \\
10 \\
30 \\
30 \\
15 \\
5\end{array}$ & $\begin{array}{l}20 \\
50 \\
50 \\
30 \\
50 \\
30 \\
50 \\
30 \\
20 \\
30\end{array}$ \\
\hline $\begin{array}{l}\text { HM0125S } \\
\text { HM0126S } \\
\text { HM0127S } \\
\text { HM0128S } \\
\text { HM0129S } \\
\text { HM0130S } \\
\text { HM0131S } \\
\text { HM0132S } \\
\text { HM0133S } \\
\text { HM0134S }\end{array}$ & $\begin{array}{l}N \\
N \\
N \\
N \\
N \\
N \\
N \\
N \\
N \\
N\end{array}$ & $\begin{array}{r}10 \\
10 \\
10 \\
<10 \\
<10 \\
<10 \\
20 \\
15 \\
<10 \\
N\end{array}$ & $\begin{array}{r}\mathbf{5 0 0} \\
\mathbf{5 0 0} \\
\mathbf{5 0 0} \\
1,000 \\
\mathbf{3 0 0} \\
\mathbf{3 0 0} \\
\mathbf{5 0 0} \\
\mathbf{5 0 0} \\
\mathbf{2 0 0} \\
\mathbf{2 0 0}\end{array}$ & $\begin{array}{l}N \\
N \\
N \\
N \\
N \\
N \\
N \\
N \\
N\end{array}$ & $\begin{array}{l}N \\
N \\
N \\
N \\
N \\
N \\
N \\
N \\
N \\
N\end{array}$ & $\begin{array}{l}N \\
N \\
N \\
N \\
N \\
N \\
N \\
N \\
N \\
N\end{array}$ & $\begin{array}{l}15 \\
15 \\
20 \\
30 \\
10 \\
10 \\
15 \\
15 \\
10 \\
10\end{array}$ & $\begin{array}{r}300 \\
150 \\
30 \\
1,000 \\
50 \\
100 \\
100 \\
200 \\
200 \\
200\end{array}$ & $\begin{array}{l}20 \\
15 \\
15 \\
20 \\
10 \\
10 \\
20 \\
30 \\
15 \\
10\end{array}$ & $\begin{array}{l}15 \\
10 \\
10 \\
30 \\
15 \\
30 \\
20 \\
20 \\
15 \\
7\end{array}$ \\
\hline $\begin{array}{l}\text { HMO135S } \\
\text { HM0136S } \\
\text { HM0137S } \\
\text { HM0138S } \\
\text { HM0139S } \\
\text { HM0140S } \\
\text { HM0141S } \\
\text { HM0142S } \\
\text { HMO1435 } \\
\text { HM0144S }\end{array}$ & $\begin{array}{l}N \\
N \\
N \\
N \\
N \\
N \\
N \\
N \\
N \\
N\end{array}$ & $\begin{array}{r}<10 \\
10 \\
15 \\
10 \\
<10 \\
10 \\
<10 \\
10 \\
<10 \\
10\end{array}$ & $\begin{array}{l}300 \\
300 \\
500 \\
300 \\
300 \\
500 \\
300 \\
500 \\
200 \\
300\end{array}$ & $\begin{array}{l}N \\
N \\
N \\
N \\
N \\
N \\
N \\
N \\
N \\
N\end{array}$ & $\begin{array}{l}N \\
N \\
N \\
N \\
N \\
N \\
N \\
N \\
N \\
N\end{array}$ & $\begin{array}{l}N \\
N \\
N \\
N \\
N \\
N \\
N \\
N \\
N \\
N\end{array}$ & $\begin{array}{r}10 \\
15 \\
30 \\
10 \\
15 \\
10 \\
15 \\
15 \\
<10 \\
15\end{array}$ & $\begin{array}{r}50 \\
500 \\
500 \\
50 \\
200 \\
50 \\
200 \\
150 \\
200 \\
50\end{array}$ & $\begin{array}{l}10 \\
20 \\
50 \\
15 \\
15 \\
15 \\
15 \\
70 \\
15 \\
10\end{array}$ & $\begin{array}{l}15 \\
20 \\
30 \\
15 \\
20 \\
15 \\
20 \\
10 \\
10 \\
15\end{array}$ \\
\hline $\begin{array}{l}\text { HMO145S } \\
\text { HM0146S } \\
\text { HM0147S } \\
\text { HM0148S } \\
\text { HM0200S } \\
\text { HM0201S } \\
\text { HM0202S } \\
\text { HM0203S } \\
\text { HM0204S } \\
\text { HM0205S }\end{array}$ & $\begin{array}{l}N \\
N \\
N \\
N \\
N \\
N \\
N \\
N \\
N \\
N\end{array}$ & $\begin{array}{r}15 \\
<10 \\
10 \\
10 \\
10 \\
<10 \\
10 \\
10 \\
<10 \\
200\end{array}$ & $\begin{array}{l}500 \\
300 \\
300 \\
300 \\
500 \\
300 \\
500 \\
500 \\
300 \\
500\end{array}$ & $\begin{array}{l}N \\
N \\
N \\
N \\
N \\
N \\
N \\
N \\
N \\
<1 \\
<1\end{array}$ & $\begin{array}{l}N \\
N \\
N \\
N \\
N \\
N \\
N \\
N \\
N \\
N\end{array}$ & $\begin{array}{l}N \\
N \\
N \\
N \\
N \\
N \\
N \\
N \\
N \\
N\end{array}$ & $\begin{array}{r}<10 \\
15 \\
15 \\
15 \\
20 \\
10 \\
20 \\
10 \\
10 \\
15\end{array}$ & $\begin{array}{r}150 \\
700 \\
100 \\
100 \\
300 \\
70 \\
100 \\
200 \\
70 \\
150\end{array}$ & $\begin{array}{l}10 \\
70 \\
20 \\
15 \\
20 \\
15 \\
20 \\
20 \\
20 \\
70\end{array}$ & $\begin{array}{l}30 \\
15 \\
20 \\
15 \\
50 \\
15 \\
20 \\
15 \\
30 \\
20\end{array}$ \\
\hline $\begin{array}{l}\text { HM0206S } \\
\text { HM0207S } \\
\text { HM0208S } \\
\text { HM0209S } \\
\text { HM0210S } \\
\text { HM0211S } \\
\text { HM0212S } \\
\text { HM0213S } \\
\text { HM0214S } \\
\text { HM0215S }\end{array}$ & $\begin{array}{l}N \\
N \\
N \\
N \\
N \\
N \\
N \\
N \\
N \\
N\end{array}$ & $\begin{array}{r}70 \\
15 \\
<10 \\
N \\
15 \\
10 \\
100 \\
20 \\
<10 \\
N\end{array}$ & $\begin{array}{l}500 \\
500 \\
300 \\
300 \\
500 \\
300 \\
300 \\
300 \\
700 \\
700\end{array}$ & $\begin{array}{r}N \\
N \\
N \\
<1 \\
N \\
N \\
<1 \\
<1 \\
N \\
N\end{array}$ & $\begin{array}{l}N \\
N \\
N \\
N \\
N \\
N \\
N \\
N \\
N \\
N\end{array}$ & $\begin{array}{l}N \\
N \\
N \\
N \\
N \\
N \\
N \\
N \\
N \\
N\end{array}$ & $\begin{array}{r}20 \\
10 \\
10 \\
<10 \\
10 \\
10 \\
10 \\
15 \\
10 \\
N\end{array}$ & $\begin{array}{r}300 \\
50 \\
150 \\
70 \\
300 \\
200 \\
300 \\
150 \\
300 \\
100\end{array}$ & $\begin{array}{l}50 \\
15 \\
30 \\
20 \\
20 \\
15 \\
20 \\
20 \\
15 \\
5\end{array}$ & $\begin{array}{l}30 \\
30 \\
30 \\
30 \\
30 \\
30 \\
30 \\
10 \\
30 \\
30\end{array}$ \\
\hline $\begin{array}{l}\text { HMO216S } \\
\text { HMO217S } \\
\text { HM0218S } \\
\text { HM0219S } \\
\text { HM0220S } \\
\text { HM0221S } \\
\text { HM0222S } \\
\text { HM0223S } \\
\text { HM0224S } \\
\text { HM0225S }\end{array}$ & $\begin{array}{l}N \\
N \\
N \\
N \\
N \\
N \\
N \\
N \\
N \\
N\end{array}$ & $\begin{array}{r}10 \\
10 \\
15 \\
<10 \\
<10 \\
10 \\
15 \\
10 \\
10 \\
15\end{array}$ & $\begin{array}{l}700 \\
300 \\
500 \\
500 \\
300 \\
300 \\
300 \\
500 \\
300 \\
500\end{array}$ & $\begin{array}{l}N \\
N \\
N \\
N \\
N \\
N \\
N \\
N \\
N \\
N\end{array}$ & $\begin{array}{l}N \\
N \\
N \\
N \\
N \\
N \\
N \\
N \\
N \\
N\end{array}$ & $\begin{array}{l}N \\
N \\
N \\
N \\
N \\
N \\
N \\
N \\
N \\
N\end{array}$ & $\begin{array}{r}10 \\
15 \\
15 \\
15 \\
20 \\
<10 \\
10 \\
15 \\
10 \\
10\end{array}$ & $\begin{array}{r}200 \\
200 \\
150 \\
300 \\
70 \\
100 \\
150 \\
150 \\
150 \\
100\end{array}$ & $\begin{array}{l}10 \\
20 \\
20 \\
20 \\
20 \\
10 \\
15 \\
15 \\
15 \\
10\end{array}$ & $\begin{array}{l}50 \\
10 \\
20 \\
20 \\
20 \\
30 \\
10 \\
20 \\
10 \\
10\end{array}$ \\
\hline $\begin{array}{l}\text { HM0226S } \\
\text { HM0227S } \\
\text { HM0228S } \\
\text { HM0229S } \\
\text { HM0230S }\end{array}$ & $\begin{array}{l}N \\
N \\
N \\
N \\
N\end{array}$ & $\begin{array}{r}10 \\
<10 \\
70 \\
<10 \\
<10\end{array}$ & $\begin{array}{l}500 \\
500 \\
500 \\
300 \\
700\end{array}$ & $\begin{array}{l}\mathbf{N} \\
\mathbf{N} \\
\mathbf{N} \\
\mathbf{N} \\
\mathbf{N}\end{array}$ & $\begin{array}{l}N \\
N \\
N \\
N \\
N\end{array}$ & $\begin{array}{l}N \\
N \\
N \\
N \\
N\end{array}$ & $\begin{array}{r}15 \\
15 \\
10 \\
<10 \\
20\end{array}$ & $\begin{array}{l}300 \\
700 \\
300 \\
300 \\
100\end{array}$ & $\begin{array}{l}15 \\
20 \\
50 \\
15 \\
20\end{array}$ & $\begin{array}{l}15 \\
30 \\
30 \\
30 \\
30\end{array}$ \\
\hline
\end{tabular}


Table 3. Analytical data for stream-sediment samples collected from the Horn Mountains area--Continued.

\begin{tabular}{|c|c|c|c|c|c|c|c|c|c|}
\hline Sample & Ge ppm-SQS & La ppm-sas & Mn ppm-SQS & Mo ppm-SQS & Nb ppm-SQS & Ni ppm-SQS & Pb ppm-SQS & Sb ppm-SQS & Sc ppm-SQS \\
\hline $\begin{array}{l}\text { HM0115S } \\
\text { HM0116S } \\
\text { HM0117S } \\
\text { HM0118S } \\
\text { HM0119S } \\
\text { HM0120S } \\
\text { HM0121S } \\
\text { HM0122S } \\
\text { HM0123S } \\
\text { HM0124S }\end{array}$ & $\begin{array}{l}N \\
N \\
N \\
N \\
N \\
N \\
N \\
N \\
N\end{array}$ & $\begin{array}{r}N \\
N \\
N \\
N \\
N \\
N \\
N \\
N \\
N \\
N\end{array}$ & $\begin{array}{r}300 \\
700 \\
500 \\
500 \\
300 \\
300 \\
700 \\
300 \\
300 \\
30\end{array}$ & $\begin{array}{l}N \\
\mathbf{5} \\
N \\
N \\
N \\
N \\
N \\
N \\
N \\
N\end{array}$ & $\begin{array}{l}N \\
N \\
N \\
N \\
N \\
N \\
N \\
N \\
N \\
N\end{array}$ & $\begin{array}{l}10 \\
20 \\
10 \\
20 \\
20 \\
10 \\
15 \\
50 \\
20 \\
7\end{array}$ & $\begin{array}{r}10 \\
15 \\
10 \\
15 \\
20 \\
<10 \\
10 \\
<10 \\
15 \\
15\end{array}$ & $\begin{array}{l}N \\
N \\
N \\
N \\
N \\
N \\
N \\
N \\
N \\
N\end{array}$ & $\begin{array}{r}7 \\
7 \\
7 \\
5 \\
5 \\
5 \\
7 \\
7 \\
<5 \\
<5\end{array}$ \\
\hline $\begin{array}{l}\text { HM0125S } \\
\text { HM0126S } \\
\text { HM0127S } \\
\text { HM0128S } \\
\text { HM0129S } \\
\text { HM0130S } \\
\text { HM0131S } \\
\text { HM0132S } \\
\text { HM0133S } \\
\text { HM0134S }\end{array}$ & $\begin{array}{l}N \\
N \\
N \\
N \\
N \\
N \\
N \\
N \\
N \\
N\end{array}$ & $\begin{array}{l}N \\
N \\
N \\
N \\
N \\
N \\
N \\
N \\
N \\
N\end{array}$ & $\begin{array}{r}300 \\
300 \\
500 \\
300 \\
200 \\
150 \\
200 \\
700 \\
70 \\
200\end{array}$ & $\begin{array}{l}N \\
N \\
N \\
N \\
N \\
N \\
N \\
<5 \\
N \\
N\end{array}$ & $\begin{array}{r}N \\
N \\
N \\
N \\
N \\
N \\
N \\
20 \\
N \\
N\end{array}$ & $\begin{array}{l}30 \\
15 \\
15 \\
50 \\
10 \\
15 \\
30 \\
30 \\
15 \\
15\end{array}$ & $\begin{array}{r}10 \\
N \\
<10 \\
<10 \\
10 \\
<10 \\
N \\
N \\
<10 \\
N\end{array}$ & $\begin{array}{l}N \\
N \\
N \\
N \\
N \\
N \\
N \\
N \\
N \\
N\end{array}$ & $\begin{array}{r}5 \\
<5 \\
5 \\
5 \\
N \\
<5 \\
5 \\
7 \\
<5 \\
<5\end{array}$ \\
\hline $\begin{array}{l}\text { HM0135S } \\
\text { HM0136S } \\
\text { HM0137S } \\
\text { HM0138S } \\
\text { HM0139S } \\
\text { HM0140S } \\
\text { HM0141S } \\
\text { HM0142S } \\
\text { HM0143S } \\
\text { HM0144S }\end{array}$ & $\begin{array}{l}N \\
N \\
N \\
N \\
N \\
N \\
N \\
N \\
N \\
N\end{array}$ & $\begin{array}{l}N \\
N \\
N \\
N \\
N \\
N \\
N \\
N \\
N \\
N\end{array}$ & $\begin{array}{r}100 \\
200 \\
500 \\
200 \\
100 \\
200 \\
300 \\
200 \\
50 \\
100\end{array}$ & $\begin{array}{l}N \\
N \\
N \\
N \\
N \\
N \\
N \\
N \\
N \\
N\end{array}$ & $\begin{array}{l}N \\
N \\
N \\
N \\
N \\
N \\
N \\
N \\
N \\
N\end{array}$ & $\begin{array}{l}15 \\
30 \\
70 \\
10 \\
30 \\
15 \\
15 \\
20 \\
10 \\
20\end{array}$ & $\begin{array}{r}N \\
N \\
<10 \\
10 \\
N \\
N \\
<10 \\
<10 \\
<10 \\
N\end{array}$ & $\begin{array}{l}N \\
N \\
N \\
N \\
N \\
N \\
N \\
N \\
N \\
N\end{array}$ & $\begin{array}{r}<5 \\
<5 \\
7 \\
<5 \\
<5 \\
<5 \\
5 \\
5 \\
<5 \\
<5\end{array}$ \\
\hline $\begin{array}{l}\text { HM0145S } \\
\text { HM0146S } \\
\text { HM0147S } \\
\text { HM0148S } \\
\text { HM0200S } \\
\text { HM0201S } \\
\text { HM0202S } \\
\text { HM0203S } \\
\text { HM0204S } \\
\text { HM0205S }\end{array}$ & $\begin{array}{l}N \\
N \\
N \\
N \\
N \\
N \\
N \\
N \\
N \\
N\end{array}$ & $\begin{array}{c}N \\
N \\
N \\
N \\
N \\
N \\
N \\
N \\
N \\
N \\
N\end{array}$ & $\begin{array}{r}70 \\
70 \\
100 \\
150 \\
300 \\
300 \\
300 \\
100 \\
300 \\
500\end{array}$ & $\begin{array}{l}N \\
N \\
N \\
N \\
N \\
N \\
N \\
N \\
N \\
N\end{array}$ & $\begin{array}{l}N \\
N \\
N \\
N \\
N \\
N \\
N \\
N \\
N \\
N\end{array}$ & $\begin{array}{l}15 \\
30 \\
30 \\
20 \\
30 \\
15 \\
50 \\
70 \\
10 \\
15\end{array}$ & $\begin{array}{r}N \\
N \\
N \\
N \\
N 10 \\
N \\
<10 \\
N \\
15 \\
20\end{array}$ & $\begin{array}{l}N \\
N \\
N \\
N \\
N \\
N \\
N \\
N \\
N\end{array}$ & $\begin{array}{r}<5 \\
<5 \\
<5 \\
<5 \\
<5 \\
<5 \\
7 \\
<5 \\
5 \\
7\end{array}$ \\
\hline $\begin{array}{l}\text { HM0206S } \\
\text { HM0207S } \\
\text { HM0208S } \\
\text { HM0209S } \\
\text { HM0210S } \\
\text { HM0211S } \\
\text { HM0212S } \\
\text { HM0213S } \\
\text { HM0214S } \\
\text { HM0215S }\end{array}$ & $\begin{array}{l}N \\
N \\
N \\
N \\
N \\
N \\
N \\
N \\
N \\
N\end{array}$ & $\begin{array}{l}N \\
N \\
N \\
N \\
N \\
N \\
N \\
N \\
N \\
N\end{array}$ & $\begin{array}{r}500 \\
150 \\
300 \\
150 \\
500 \\
150 \\
150 \\
200 \\
200 \\
70\end{array}$ & $\begin{array}{l}N \\
N \\
N \\
N \\
N \\
N \\
N \\
N \\
N \\
N\end{array}$ & $\begin{array}{l}N \\
N \\
N \\
N \\
N \\
N \\
N \\
N \\
N \\
N\end{array}$ & $\begin{array}{l}30 \\
10 \\
10 \\
10 \\
10 \\
10 \\
15 \\
15 \\
10 \\
5\end{array}$ & $\begin{array}{r}10 \\
15 \\
15 \\
10 \\
10 \\
20 \\
70 \\
<10 \\
10 \\
15\end{array}$ & $\begin{array}{l}N \\
N \\
N \\
N \\
N \\
N \\
N \\
N \\
N \\
N\end{array}$ & $\begin{array}{r}10 \\
5 \\
5 \\
5 \\
5 \\
<5 \\
5 \\
5 \\
<5 \\
N\end{array}$ \\
\hline $\begin{array}{l}\text { HM0216S } \\
\text { HM0217S } \\
\text { HM0218S } \\
\text { HM0219S } \\
\text { HM0220S } \\
\text { HM0221S } \\
\text { HM0222S } \\
\text { HM0223S } \\
\text { HM0224S } \\
\text { HM0225S }\end{array}$ & $\begin{array}{l}N \\
N \\
N \\
N \\
N \\
N \\
N \\
N \\
N \\
N\end{array}$ & $\begin{array}{r}<50 \\
N \\
N \\
N \\
N \\
<50 \\
<50 \\
<50 \\
N \\
N\end{array}$ & $\begin{array}{r}70 \\
200 \\
300 \\
100 \\
200 \\
100 \\
150 \\
200 \\
100 \\
200\end{array}$ & $\begin{array}{l}\mathbf{N} \\
\mathbf{N} \\
\mathbf{N} \\
\mathbf{N} \\
\mathbf{N} \\
\mathbf{N} \\
\mathbf{N} \\
\mathbf{N} \\
\mathbf{N} \\
\mathbf{N}\end{array}$ & $\begin{array}{l}\mathbf{N} \\
\mathbf{N} \\
\mathbf{N} \\
\mathbf{N} \\
\mathbf{N} \\
\mathbf{N} \\
\mathbf{N} \\
\mathbf{N} \\
\mathbf{N} \\
\mathbf{N}\end{array}$ & $\begin{array}{l}15 \\
15 \\
30 \\
20 \\
50 \\
15 \\
20 \\
30 \\
20 \\
30\end{array}$ & $\begin{array}{r}20 \\
<10 \\
N \\
<10 \\
N \\
<10 \\
N \\
N \\
N \\
N\end{array}$ & $\begin{array}{l}N \\
N \\
N \\
N \\
N \\
N \\
N \\
N \\
N \\
N\end{array}$ & $\begin{array}{r}<5 \\
5 \\
5 \\
<5 \\
5 \\
<5 \\
<5 \\
5 \\
<5 \\
<5\end{array}$ \\
\hline $\begin{array}{l}\text { HM0226S } \\
\text { HM0227S } \\
\text { HM0228S } \\
\text { HM0229S } \\
\text { HM0230S }\end{array}$ & $\begin{array}{l}N \\
N \\
N \\
N \\
N\end{array}$ & $\begin{array}{l}\mathbf{N} \\
\mathbf{N} \\
\mathbf{N} \\
\mathbf{N} \\
\mathbf{N}\end{array}$ & $\begin{array}{r}100 \\
100 \\
500 \\
150 \\
1,000\end{array}$ & $\begin{array}{l}N \\
N \\
N \\
N \\
N\end{array}$ & $\begin{array}{l}N \\
N \\
N \\
N \\
N\end{array}$ & $\begin{array}{l}30 \\
50 \\
20 \\
15 \\
20\end{array}$ & $\begin{array}{r}N \\
N \\
10 \\
<10 \\
<10\end{array}$ & $\begin{array}{l}N \\
N \\
N \\
N \\
N\end{array}$ & $\begin{array}{r}5 \\
<5 \\
5 \\
<5 \\
5\end{array}$ \\
\hline
\end{tabular}


Table 3. Analytical data for stream-sediment samples collected from the Horn Mountains area--Continued.

\begin{tabular}{|c|c|c|c|c|c|c|c|c|c|c|}
\hline Sample & Sn ppm-Sas & sr ppm-SQS & Th ppm-SQS & V ppm-SQS & W ppm-sas & Y ppm-sas & Zn ppm-sas & S Zr ppm-sas & Se ppm-MAS & Te ppm-AAS \\
\hline $\begin{array}{l}\text { HMO115S } \\
\text { HMO116S } \\
\text { HMO117S } \\
\text { HMO118S } \\
\text { HMO119S } \\
\text { HMO120S } \\
\text { HM0121S } \\
\text { HMO122S } \\
\text { HMO123S } \\
\text { HMO124S }\end{array}$ & $\begin{array}{l}N \\
N \\
N \\
N \\
N \\
N \\
N \\
N \\
N \\
N\end{array}$ & $\begin{array}{r}N \\
<100 \\
<100 \\
<100 \\
N \\
N \\
<100 \\
N \\
N \\
N\end{array}$ & $\begin{array}{l}N \\
N \\
N \\
N \\
N \\
N \\
N \\
N \\
N \\
N\end{array}$ & $\begin{array}{l}50 \\
50 \\
30 \\
50 \\
50 \\
20 \\
70 \\
70 \\
70 \\
20\end{array}$ & $\begin{array}{l}N \\
N \\
N \\
N \\
N \\
N \\
N \\
N \\
N \\
N\end{array}$ & $\begin{array}{r}10 \\
20 \\
20 \\
<10 \\
<10 \\
<10 \\
10 \\
<10 \\
N \\
N\end{array}$ & $\begin{array}{l}N \\
N \\
N \\
N \\
N \\
N \\
N \\
N \\
N \\
N\end{array}$ & $\begin{array}{r}50 \\
70 \\
100 \\
70 \\
30 \\
50 \\
200 \\
70 \\
150 \\
30\end{array}$ & $\begin{array}{r}.8 \\
1.2 \\
.5 \\
.5 \\
1.3 \\
.3 \\
.2 \\
.1 \\
.6 \\
.3\end{array}$ & $\begin{array}{l}\dot{N}^{2} \\
N \\
N \\
N \\
N \\
N \\
N \\
N \\
N\end{array}$ \\
\hline $\begin{array}{l}\text { HMO125S } \\
\text { HMO126S } \\
\text { HM0127S } \\
\text { HMO128S } \\
\text { HM0129S } \\
\text { HM0130S } \\
\text { HM0131S } \\
\text { HM0132S } \\
\text { HM0133S } \\
\text { HMO134S }\end{array}$ & $\begin{array}{l}N \\
N \\
N \\
N \\
N \\
N \\
N \\
N \\
N \\
N\end{array}$ & $\begin{array}{r}<100 \\
N \\
N \\
N \\
N \\
N \\
N \\
N \\
N \\
N \\
N \\
N\end{array}$ & $\begin{array}{l}N \\
N \\
N \\
N \\
N \\
N \\
N \\
N \\
N \\
N\end{array}$ & $\begin{array}{r}50 \\
30 \\
50 \\
100 \\
30 \\
30 \\
70 \\
50 \\
50 \\
50\end{array}$ & $\begin{array}{l}N \\
N \\
N \\
N \\
N \\
N \\
N \\
N \\
N \\
N\end{array}$ & $\begin{array}{r}<10 \\
<10 \\
<10 \\
N \\
N \\
<10 \\
10 \\
10 \\
N \\
N\end{array}$ & $\begin{array}{l}N \\
N \\
N \\
N \\
N \\
N \\
N \\
N \\
N \\
N\end{array}$ & $\begin{array}{r}70 \\
70 \\
50 \\
50 \\
30 \\
200 \\
100 \\
100 \\
30 \\
30\end{array}$ & $\begin{array}{l}.4 \\
.2 \\
.3 \\
.2 \\
.3 \\
.1 \\
.3 \\
.4 \\
.3 \\
.2\end{array}$ & $\begin{array}{l}N \\
N \\
.05 \\
N \\
N \\
N \\
N \\
N \\
N \\
N\end{array}$ \\
\hline $\begin{array}{l}\text { HMO135S } \\
\text { HMO136S } \\
\text { HMO137S } \\
\text { HMO138S } \\
\text { HMO139S } \\
\text { HMO140S } \\
\text { HM0141S } \\
\text { HMO142S } \\
\text { HM0143S } \\
\text { HMO144S }\end{array}$ & $\begin{array}{l}N \\
N \\
N \\
N \\
N \\
N \\
N \\
N \\
N \\
N\end{array}$ & $\begin{array}{l}N \\
N \\
N \\
N \\
N \\
N \\
N \\
N \\
N \\
N\end{array}$ & $\begin{array}{l}N \\
N \\
N \\
N \\
N \\
N \\
N \\
N \\
N \\
N\end{array}$ & $\begin{array}{r}30 \\
100 \\
100 \\
30 \\
50 \\
50 \\
50 \\
50 \\
30 \\
70\end{array}$ & $\begin{array}{l}N \\
N \\
N \\
N \\
N \\
N \\
N \\
N \\
N \\
N\end{array}$ & $\begin{array}{r}N \\
<10 \\
10 \\
N \\
N \\
<10 \\
<10 \\
<10 \\
N \\
N\end{array}$ & $\begin{array}{l}N \\
N \\
N \\
N \\
N \\
N \\
N \\
N \\
N \\
N\end{array}$ & $\begin{array}{r}30 \\
200 \\
70 \\
20 \\
200 \\
70 \\
50 \\
50 \\
30 \\
70\end{array}$ & $\begin{array}{l}.1 \\
.2 \\
.3 \\
.5 \\
.2 \\
.3 \\
.3 \\
.3 \\
.1 \\
.1\end{array}$ & $\begin{array}{l}N \\
N \\
N \\
N \\
N \\
N \\
N \\
N \\
N \\
.05\end{array}$ \\
\hline $\begin{array}{l}\text { HM0145S } \\
\text { HMO146S } \\
\text { HM0147S } \\
\text { HM0148S } \\
\text { HM0200S } \\
\text { HM0201S } \\
\text { HM0202S } \\
\text { HM0203S } \\
\text { HM0204S } \\
\text { HM0205S }\end{array}$ & $\begin{array}{l}N \\
N \\
N \\
N \\
N \\
N \\
N \\
N \\
N \\
N\end{array}$ & $<\begin{array}{r}N \\
N \\
N \\
N \\
N \\
N \\
N \\
N \\
N\end{array}$ & $\begin{array}{l}N \\
N \\
N \\
N \\
N \\
N \\
N \\
N \\
N \\
N\end{array}$ & $\begin{array}{r}20 \\
70 \\
70 \\
50 \\
70 \\
50 \\
100 \\
70 \\
30 \\
30\end{array}$ & $\begin{array}{r}N \\
N \\
N \\
<20 \\
N \\
N \\
N \\
N \\
N \\
N\end{array}$ & $\begin{array}{r}N \\
N \\
N \\
<10 \\
<10 \\
N \\
15 \\
<10 \\
10 \\
15\end{array}$ & $\begin{array}{l}N \\
N \\
N \\
N \\
N \\
N \\
N \\
N \\
N \\
N\end{array}$ & $\begin{array}{r}30 \\
100 \\
50 \\
70 \\
70 \\
70 \\
150 \\
500 \\
50 \\
50\end{array}$ & $\begin{array}{r}.4 \\
.3 \\
.5 \\
.1 \\
.4 \\
.4 \\
.2 \\
.3 \\
1.5 \\
.9\end{array}$ & $\begin{array}{l}N \\
N \\
.05 \\
N \\
N \\
N \\
N \\
N \\
N \\
.6\end{array}$ \\
\hline $\begin{array}{l}\text { HM0206S } \\
\text { HM0207S } \\
\text { HM0208S } \\
\text { HM0209S } \\
\text { HM0210S } \\
\text { HM0211S } \\
\text { HM0212S } \\
\text { HM0213S } \\
\text { HM0214S } \\
\text { HM0215S }\end{array}$ & $\begin{array}{l}N \\
N \\
N \\
N \\
N \\
N \\
N \\
N \\
N \\
N\end{array}$ & $\begin{array}{r}<100 \\
N \\
N \\
N \\
<100 \\
N \\
N \\
N \\
<100 \\
N\end{array}$ & $\begin{array}{l}N \\
N \\
N \\
N \\
N \\
N \\
N \\
N \\
N \\
N\end{array}$ & $\begin{array}{l}70 \\
30 \\
50 \\
30 \\
50 \\
30 \\
30 \\
50 \\
30 \\
15\end{array}$ & $\begin{array}{r}N \\
N \\
N \\
N \\
N \\
<20 \\
N \\
N \\
N \\
N\end{array}$ & $\begin{array}{r}10 \\
<10 \\
10 \\
10 \\
<10 \\
N \\
<10 \\
<10 \\
N \\
N\end{array}$ & $\begin{array}{l}N \\
N \\
N \\
N \\
N \\
N \\
N \\
N \\
N \\
N\end{array}$ & $\begin{array}{r}150 \\
50 \\
30 \\
50 \\
50 \\
20 \\
50 \\
50 \\
30 \\
20\end{array}$ & $\begin{array}{l}.6 \\
1.2 \\
1.1 \\
.9 \\
.5 \\
.9 \\
2 \\
1.6 \\
.2 \\
.1\end{array}$ & $\begin{array}{l}.05 \\
N \\
N \\
N \\
N \\
.2 \\
.1 \\
N \\
N\end{array}$ \\
\hline $\begin{array}{l}\text { HM0216S } \\
\text { HMO217S } \\
\text { HM0218S } \\
\text { HM0219S } \\
\text { HM0220S } \\
\text { HMO221S } \\
\text { HM0222S } \\
\text { HM0223S } \\
\text { HM0224S } \\
\text { HM0225S }\end{array}$ & $\begin{array}{l}N \\
N \\
N \\
N \\
N \\
N \\
N \\
N \\
N \\
N\end{array}$ & $\begin{array}{l}N \\
N \\
N \\
N \\
N \\
N \\
N \\
N \\
N \\
N\end{array}$ & $\begin{array}{l}N \\
N \\
N \\
N \\
N \\
N \\
N \\
N \\
N \\
N\end{array}$ & $\begin{array}{r}30 \\
50 \\
70 \\
50 \\
100 \\
50 \\
50 \\
70 \\
70 \\
70\end{array}$ & $\begin{array}{l}N \\
N \\
N \\
N \\
N \\
N \\
N \\
N \\
N \\
N\end{array}$ & $\begin{array}{r}N \\
<10 \\
10 \\
<10 \\
<10 \\
<10 \\
N \\
<10 \\
<10 \\
<10\end{array}$ & $\begin{array}{l}N \\
N \\
N \\
N \\
N \\
N \\
N \\
N \\
N \\
N\end{array}$ & $\begin{array}{r}30 \\
70 \\
150 \\
70 \\
200 \\
300 \\
150 \\
70 \\
70 \\
300\end{array}$ & $\begin{array}{l}.3 \\
.3 \\
.1 \\
.1 \\
.2 \\
<.1 \\
.2 \\
.2 \\
.2 \\
.1\end{array}$ & $\begin{array}{l}N \\
.2 \\
N \\
N \\
.05 \\
N \\
N \\
N \\
N\end{array}$ \\
\hline $\begin{array}{l}\text { HM0226s } \\
\text { HM0227S } \\
\text { HM02285 } \\
\text { HM0229S } \\
\text { HM0230S }\end{array}$ & $\begin{array}{l}N \\
N \\
N \\
N \\
N\end{array}$ & $\begin{array}{r}N \\
N \\
N \\
N \\
<100\end{array}$ & $\begin{array}{l}N \\
N \\
N \\
N \\
N\end{array}$ & $\begin{array}{l}70 \\
70 \\
50 \\
50 \\
50\end{array}$ & $\begin{array}{l}N \\
N \\
N \\
N\end{array}$ & $\begin{array}{r}<10 \\
10 \\
<10 \\
N \\
10\end{array}$ & $\begin{array}{l}N \\
N \\
N \\
N\end{array}$ & $\begin{array}{r}70 \\
50 \\
70 \\
30 \\
100\end{array}$ & $\begin{array}{l}.3 \\
.2 \\
.5 \\
.2 \\
.4\end{array}$ & $\begin{array}{l}N \\
N \\
N \\
N \\
N\end{array}$ \\
\hline
\end{tabular}


Table 3. Analytical data for stream-sediment samples collected from the Horn Mountains area--Continued.

\begin{tabular}{|c|c|c|c|c|c|c|c|c|c|c|}
\hline Sample & Tl ppm-AAS & AU ppm-AAS & Hg ppm-MAS & W ppm-VS & F \%-SIE & Pt ppb-ICP & Pd ppb-ICP & Rh ppb-ICP & Ru ppb-ICP & Ir ppb-ICP \\
\hline $\begin{array}{l}\text { HM0115S } \\
\text { HM0116S } \\
\text { HM0117S } \\
\text { HM0118S } \\
\text { HM0119S } \\
\text { HM0120S } \\
\text { HM0121S } \\
\text { HM0122S } \\
\text { HM0123S } \\
\text { HM0124S }\end{array}$ & $\begin{array}{l}.65 \\
.2 \\
.35 \\
.4 \\
.45 \\
.25 \\
.4 \\
.2 \\
.3 \\
.4\end{array}$ & $\begin{array}{l}<.002 \\
N \\
N \\
N \\
.002 \\
<.002 \\
N \\
N \\
N \\
N\end{array}$ & $\begin{array}{l}.12 \\
.07 \\
.11 \\
.65 \\
.23 \\
1.32 \\
.85 \\
.05 \\
.56 \\
.18\end{array}$ & $\begin{array}{l}3 \\
4 \\
7 \\
2 \\
2 \\
1 \\
3 \\
1 \\
4 \\
4\end{array}$ & $\begin{array}{l}.11 \\
.06 \\
.04 \\
.04 \\
.06 \\
.05 \\
.05 \\
.04 \\
.08 \\
.05\end{array}$ & $\begin{array}{l}<2 \\
<.5 \\
<.5 \\
<1 \\
<1 \\
<.5 \\
<1 \\
<1 \\
<1\end{array}$ & $\begin{array}{l}<.5 \\
<.5 \\
<.5 \\
<.5 \\
<.5 \\
<.5 \\
<.5 \\
<.5 \\
-. . \\
<.5\end{array}$ & $\begin{array}{l}<.5 \\
<.5 \\
<.5 \\
<.5 \\
<.5 \\
<.5 \\
<.5 \\
<.5 \\
-. .\end{array}$ & $\begin{array}{r}<1 \\
<.5 \\
<.5 \\
<.5 \\
.7 \\
<.5 \\
.6 \\
.6 \\
-. \\
.5\end{array}$ & $\begin{array}{l}<.5 \\
<.5 \\
<.5 \\
<.5 \\
<.5 \\
<.5 \\
<.5 \\
<.5 \\
-. . \\
<.5\end{array}$ \\
\hline $\begin{array}{l}\text { HM0125S } \\
\text { HM0126S } \\
\text { HM0127S } \\
\text { HM0128S } \\
\text { HM0129S } \\
\text { HM0130S } \\
\text { HM0131S } \\
\text { HM0132S } \\
\text { HM0133S } \\
\text { HM0134S }\end{array}$ & $\begin{array}{l}.4 \\
.2 \\
.45 \\
.3 \\
.2 \\
.35 \\
.25 \\
.25 \\
.3 \\
.3\end{array}$ & $\begin{array}{l}N \\
N \\
N \\
N \\
N \\
N \\
N \\
.004 \\
N \\
N\end{array}$ & $\begin{array}{l}.35 \\
2.4 \\
.08 \\
6.7 \\
.07 \\
1.25 \\
.07 \\
.43 \\
.1 \\
.18\end{array}$ & $\begin{array}{l}1 \\
1 \\
N \\
3 \\
N \\
1 \\
2 \\
2 \\
1 \\
1\end{array}$ & $\begin{array}{l}.08 \\
.04 \\
.05 \\
.08 \\
.04 \\
.03 \\
.05 \\
.05 \\
.04 \\
.04\end{array}$ & $\begin{array}{l}<1.5 \\
<1 \\
<1 \\
<.5 \\
<1.5 \\
<.5 \\
<.5 \\
<.5\end{array}$ & $\begin{array}{l}<.5 \\
<.5 \\
.8 \\
<.5 \\
<.5 \\
<.5 \\
<.5 \\
<.5 \\
.5 \\
<.5\end{array}$ & $\begin{array}{l}<.5 \\
<.5 \\
<.6 \\
<.5 \\
<.5 \\
<.5 \\
<.5 \\
<.5 \\
<.5 \\
<.5\end{array}$ & $\begin{array}{l}<.5 \\
<.5 \\
<.6 \\
<.5 \\
<.5 \\
<.5 \\
<.5 \\
<.5 \\
<.5 \\
4\end{array}$ & $\begin{array}{l}<.5 \\
<.5 \\
<.6 \\
<.5 \\
<.5 \\
<.5 \\
<.5 \\
<.5 \\
<.5 \\
.9\end{array}$ \\
\hline $\begin{array}{l}\text { HM0135S } \\
\text { HM0136S } \\
\text { HM0137S } \\
\text { HM0138S } \\
\text { HM0139S } \\
\text { HM0140S } \\
\text { HM0141S } \\
\text { HM0142S } \\
\text { HM0143S } \\
\text { HM0144S }\end{array}$ & $\begin{array}{l}.4 \\
.3 \\
.4 \\
.2 \\
.25 \\
.3 \\
.2 \\
.2 \\
.2 \\
.35\end{array}$ & $\begin{array}{l}N \\
.006 \\
N \\
N \\
N \\
N \\
N \\
.028 \\
.006 \\
N\end{array}$ & $\begin{array}{l}.06 \\
.04 \\
.07 \\
.39 \\
.09 \\
1.54 \\
.09 \\
.28 \\
.11 \\
.64\end{array}$ & $\begin{array}{l}2 \\
2 \\
1 \\
3 \\
1 \\
1 \\
1 \\
1 \\
2 \\
2\end{array}$ & $\begin{array}{l}.05 \\
.04 \\
.05 \\
.05 \\
.04 \\
.04 \\
.04 \\
.04 \\
.04 \\
.04\end{array}$ & $\begin{array}{l}<1 \\
<1 \\
1 \\
<.5 \\
<.5 \\
<1 \\
<.5 \\
<.5 \\
<.5 \\
<1\end{array}$ & $\begin{array}{l}<.5 \\
<.5 \\
.5 \\
.6 \\
<.5 \\
<.5 \\
.5 \\
<.5 \\
<.5 \\
<.5\end{array}$ & $\begin{array}{l}<.5 \\
<.5 \\
<.5 \\
<.5 \\
<.5 \\
<.5 \\
<.5 \\
<.5 \\
<.5 \\
<.5\end{array}$ & $\begin{array}{l}<.5 \\
<.5 \\
<.5 \\
<.5 \\
<.5 \\
<.5 \\
<.5 \\
<.5 \\
<.5 \\
<.5\end{array}$ & $\begin{array}{l}<.5 \\
<.5 \\
<.5 \\
<.5 \\
<.5 \\
<.5 \\
<.5 \\
<.5 \\
<.5 \\
<.5\end{array}$ \\
\hline $\begin{array}{l}\text { HM0145S } \\
\text { HM0146S } \\
\text { HM0147S } \\
\text { HM0148S } \\
\text { HM0200S } \\
\text { HM0201S } \\
\text { HM0202S } \\
\text { HM0203S } \\
\text { HM0204S } \\
\text { HM0205S }\end{array}$ & $\begin{array}{l}.2 \\
.35 \\
.25 \\
.15 \\
.15 \\
.2 \\
.4 \\
.15 \\
.45 \\
.45\end{array}$ & $\begin{array}{l}<.002 \\
.028 \\
<.002 \\
N \\
N \\
N \\
N \\
.012 \\
N \\
.01\end{array}$ & $\begin{array}{l}3.4 \\
.88 \\
.05 \\
.04 \\
.11 \\
.04 \\
.68 \\
.12 \\
.53 \\
.31\end{array}$ & $\begin{array}{l}2 \\
2 \\
1 \\
2 \\
3 \\
2 \\
3 \\
3 \\
2 \\
5\end{array}$ & $\begin{array}{l}.04 \\
.04 \\
.06 \\
.04 \\
.05 \\
.05 \\
.05 \\
.06 \\
.05 \\
.09\end{array}$ & $\begin{array}{l}<.5 \\
<1 \\
<.5 \\
<.5 \\
<1 \\
<.5 \\
<1 \\
<1 \\
<1 \\
<.5\end{array}$ & $\begin{array}{l}<.5 \\
<.5 \\
.7 \\
<.5 \\
<.5 \\
<.5 \\
.6 \\
.9 \\
<.5 \\
.8\end{array}$ & $\begin{array}{l}<.5 \\
<.5 \\
<.5 \\
<.5 \\
<.5 \\
<.5 \\
<.5 \\
<.5 \\
<.5 \\
<.5\end{array}$ & $\begin{array}{l}<.5 \\
.5 \\
<.5 \\
<.5 \\
<.5 \\
<.5 \\
<.5 \\
.5 \\
<.5 \\
<.5\end{array}$ & $\begin{array}{l}<.5 \\
<.5 \\
<.5 \\
<.5 \\
<.5 \\
<.5 \\
<.5 \\
<.5 \\
<.5 \\
<.5\end{array}$ \\
\hline $\begin{array}{l}\text { HM0206S } \\
\text { HM0207S } \\
\text { HM0208S } \\
\text { HM0209S } \\
\text { HM0210S } \\
\text { HM0211S } \\
\text { HM0212S } \\
\text { HM0213S } \\
\text { HM0214S } \\
\text { HM0215S }\end{array}$ & $\begin{array}{l}.25 \\
.35 \\
.2 \\
.45 \\
.4 \\
.3 \\
.25 \\
.45 \\
.25 \\
.3\end{array}$ & $\begin{array}{l}N \\
<.002 \\
.006 \\
N \\
N \\
.004 \\
.018 \\
N \\
N \\
N\end{array}$ & $\begin{array}{l}2.13 \\
.05 \\
.09 \\
.19 \\
.39 \\
.33 \\
4^{.14} \\
.64 \\
8^{.64}\end{array}$ & $\begin{array}{r}2 \\
16 \\
2 \\
2 \\
2 \\
2 \\
2 \\
1 \\
1 \\
3\end{array}$ & $\begin{array}{l}.05 \\
.06 \\
.06 \\
.05 \\
.05 \\
.08 \\
.09 \\
.05 \\
.05 \\
.04\end{array}$ & $\begin{array}{l}<.5 \\
<.5 \\
<.5 \\
<1 \\
2 \\
<.5 \\
<.5 \\
<1 \\
<.5\end{array}$ & $\begin{array}{l}<.5 \\
<.5 \\
.5 \\
<.5 \\
<.5 \\
<.5 \\
<.5 \\
<.5 \\
<.5 \\
<.5\end{array}$ & $\begin{array}{l}<.5 \\
<.5 \\
<.5 \\
<.5 \\
<.5 \\
<.5 \\
<.5 \\
<.5 \\
<.5 \\
<.5\end{array}$ & $\begin{array}{l}<.5 \\
<.5 \\
<.5 \\
.6 \\
<.5 \\
<.5 \\
<.5 \\
<.5 \\
<.5 \\
.6\end{array}$ & $\begin{array}{l}<.5 \\
<.5 \\
<.5 \\
<.5 \\
<.5 \\
<.5 \\
<.5 \\
<.5 \\
<.5 \\
<.5\end{array}$ \\
\hline $\begin{array}{l}\text { HM0216S } \\
\text { HM0217S } \\
\text { HM0218S } \\
\text { HM0219S } \\
\text { HM0220S } \\
\text { HM0221S } \\
\text { HM0222S } \\
\text { HM0223S } \\
\text { HM0224S } \\
\text { HM0225S }\end{array}$ & $\begin{array}{l}.3 \\
.2 \\
.1 \\
.35 \\
.2 \\
.45 \\
.25 \\
.15 \\
.3 \\
.35\end{array}$ & $\begin{array}{l}N \\
N \\
N \\
N \\
N \\
N \\
N \\
N \\
N \\
N\end{array}$ & $\begin{array}{l}.2 \\
.07 \\
.41 \\
.04 \\
.02 \\
.02 \\
.3 \\
.13 \\
.05 \\
.47\end{array}$ & $\begin{array}{l}1 \\
N \\
1 \\
1 \\
2 \\
3 \\
1 \\
1 \\
2 \\
2\end{array}$ & $\begin{array}{l}.06 \\
.04 \\
.04 \\
.04 \\
.04 \\
.05 \\
.04 \\
.04 \\
.05 \\
.04\end{array}$ & $\begin{array}{l}<.5 \\
<.5 \\
<.5 \\
<1.7 \\
<1.7 \\
<.5 \\
<.5 \\
<1 \\
<1\end{array}$ & $\begin{array}{l}<.5 \\
<.5 \\
<.5 \\
<.5 \\
<.5 \\
<.5 \\
<.5 \\
<.5 \\
<.5 \\
<.5\end{array}$ & $\begin{array}{l}<.5 \\
<.5 \\
<.5 \\
<.5 \\
<.5 \\
<.5 \\
<.5 \\
<.5 \\
<.5 \\
<.5\end{array}$ & $\begin{array}{l}<.5 \\
<.5 \\
<.5 \\
.5 \\
<.5 \\
<.5 \\
<.5 \\
<.5 \\
<.5 \\
<.5\end{array}$ & $\begin{array}{l}<.5 \\
<.5 \\
<.5 \\
<.5 \\
<.5 \\
<.5 \\
<.5 \\
<.5 \\
<.5 \\
<.5\end{array}$ \\
\hline $\begin{array}{l}\text { HM0226S } \\
\text { HM0227S } \\
\text { HM0228S } \\
\text { HM0229S } \\
\text { HM0230S }\end{array}$ & $\begin{array}{l}.25 \\
.3 \\
.25 \\
.4 \\
.4\end{array}$ & $\begin{array}{l}N \\
N \\
.1 \\
.002\end{array}$ & $\begin{array}{l}.04 \\
.22 \\
.18 \\
.65 \\
.14\end{array}$ & $\begin{array}{l}2 \\
3 \\
2 \\
3 \\
3\end{array}$ & $\begin{array}{l}.06 \\
.04 \\
.06 \\
.05 \\
.04\end{array}$ & $\begin{array}{l}<.5 \\
<1 \\
<.5 \\
<1\end{array}$ & $\begin{array}{r}<.5 \\
<.5 \\
.5 \\
<.5 \\
.8\end{array}$ & $\begin{array}{l}<.5 \\
<.5 \\
<.5 \\
<.5 \\
<.5\end{array}$ & $\begin{array}{l}<.5 \\
<.5 \\
<.5 \\
<.5 \\
<.5\end{array}$ & $\begin{array}{l}<.5 \\
<.5 \\
<.5 \\
<.5 \\
<.5\end{array}$ \\
\hline
\end{tabular}


Table 3. Analytical data for stream-sediment samples collected from the Horn Mountains area--Continued.

\begin{tabular}{|c|c|c|c|c|c|c|c|c|c|c|}
\hline Sample & Latitude & Longi tude & Ag ppm-ICP & As $p p m-I C P$ & Au ppm-ICP & Bi ppm-ICP & Cd ppm-ICP & Cu ppm-I CP & Mo ppm-ICP & $\mathrm{Pb} p p m-I C P$ \\
\hline $\begin{array}{l}\text { HM0231S } \\
\text { HM0232S } \\
\text { HM0233S } \\
\text { HM0234S } \\
\text { HM0235S } \\
\text { HM0236S } \\
\text { HM0237S } \\
\text { HM0238S }\end{array}$ & $\begin{array}{rrr}61 & 53 & 4 \\
61 & 54 & 56 \\
61 & 51 & 11 \\
61 & 47 & 2 \\
61 & 48 & 1 \\
61 & 43 & 34 \\
61 & 41 & 51 \\
61 & 41 & 41\end{array}$ & $\begin{array}{rrr}158 & 37 & 54 \\
158 & 54 & 51 \\
158 & 48 & 33 \\
158 & 49 & 23 \\
158 & 58 & 34 \\
158 & 53 & 13 \\
158 & 52 & 1 \\
158 & 46 & 46\end{array}$ & $\begin{array}{l}.086 \\
N \\
N \\
N \\
N \\
N \\
N \\
N\end{array}$ & $\begin{array}{l}5.5 \\
5.3 \\
4.2 \\
3.5 \\
5.7 \\
6 \\
4.7 \\
6.2\end{array}$ & $\begin{array}{l}N \\
N \\
N \\
N \\
N \\
N \\
N \\
N\end{array}$ & $\begin{array}{l}N \\
N \\
N \\
N \\
N \\
N \\
N \\
N\end{array}$ & $\begin{array}{l}.22 \\
.15 \\
.12 \\
.13 \\
.1 \\
.15 \\
.094 \\
.11\end{array}$ & $\begin{array}{l}12 \\
14 \\
11 \\
10 \\
10 \\
13 \\
13 \\
13\end{array}$ & $\begin{array}{l}.34 \\
.54 \\
.44 \\
.5 \\
.44 \\
.63 \\
.52 \\
.5\end{array}$ & $\begin{array}{l}7.9 \\
7.2 \\
6.5 \\
6 \\
6.2 \\
7.2 \\
6.3 \\
6.1\end{array}$ \\
\hline
\end{tabular}

Sample Sb ppm-ICP Zn ppm-ICP Ca \%-SQS fe \%-SQS Mg \%-SQS Na \%-SQS P \%-SaS Ti \%-SQS Ag ppm-SQS As ppm-SQS

\begin{tabular}{|c|c|c|c|c|c|c|c|c|c|c|}
\hline $\begin{array}{l}\text { HM0231S } \\
\text { HM0232S } \\
\text { HM0233S } \\
\text { HM0234S } \\
\text { HM0235S } \\
\text { HM0236S } \\
\text { HM0237S } \\
\text { HM0238S }\end{array}$ & $\begin{array}{l}N \\
N \\
N \\
N \\
N \\
N \\
N \\
N\end{array}$ & $\begin{array}{l}62 \\
65 \\
71 \\
72 \\
74 \\
71 \\
82 \\
79\end{array}$ & $\begin{array}{l}.2 \\
.15 \\
.1 \\
.1 \\
.1 \\
.15 \\
.05 \\
.07\end{array}$ & $\begin{array}{l}5 \\
2 \\
3 \\
5 \\
3 \\
5 \\
2 \\
2\end{array}$ & $\begin{array}{l}.5 \\
.3 \\
.5 \\
.3 \\
.5 \\
.5 \\
.3 \\
3\end{array}$ & $\begin{array}{l}3 \\
2 \\
2 \\
2 \\
2 \\
3 \\
1.5\end{array}$ & $\begin{array}{l}N \\
N \\
N \\
N \\
N \\
N \\
N\end{array}$ & $\begin{array}{l}.5 \\
.3 \\
.3 \\
.5 \\
.2 \\
.7 \\
.2 \\
.2\end{array}$ & $\begin{array}{l}N \\
N \\
N \\
N \\
N \\
N \\
N \\
N\end{array}$ & $\begin{array}{l}N \\
N \\
N \\
N \\
N \\
N \\
N \\
N\end{array}$ \\
\hline
\end{tabular}

Sample AU ppm-SQS B ppm-SQS Ba ppm-SQS Be ppm-SQS Bi ppm-SQS Cd ppm-SQS Co ppm-SQS Cr ppm-SQS Cu ppm-SQS Ga ppm-SQS

$\begin{array}{lrrr}\text { HM0231S } & N & <10 & 500 \\ \text { HM0232S } & N & <10 & 500 \\ \text { HM0233S } & N & 10 & 300 \\ \text { HM0234S } & N & <10 & 500 \\ \text { HM0235S } & N & N & 500 \\ \text { HM0236S } & N & 10 & 500 \\ \text { HM0237S } & N & 10 & 300 \\ \text { HM0238S } & N & <10 & 300\end{array}$

$\begin{array}{ll}N & N \\ N & N \\ N & N \\ N & N \\ N & N \\ N & N \\ N & N \\ N & N\end{array}$

$N$
$N$
$N$
$N$
$N$
$N$
$N$
$N$

$\begin{array}{rr}20 & 150 \\ 15 & 100 \\ 15 & 70 \\ 20 & 150 \\ 10 & 500 \\ 20 & 100 \\ <10 & 200 \\ 15 & 70\end{array}$

$\begin{array}{ll}20 & 50 \\ 20 & 15 \\ 15 & 20 \\ 20 & 30 \\ 10 & 20 \\ 30 & 30 \\ 15 & 20 \\ 15 & 7\end{array}$

Sample

HM0231S HM0232S

HM0233S

HM0234S

HM0235S

HM0236S

HM0237S

HM0238S

Ge ppm-sas La ppm-sas Mn ppm-sas Mo ppm-SaS

Mb ppon-sas

Ni pom-ses

$\mathrm{Pb}$ ppm-s

Sb ppm-SaS Sc ppm-SaS

Sample

$N$
$N$
$N$
$N$
$N$
$N$
$N$
$N$

$\begin{array}{rr}N & 300 \\ N & 300 \\ N & 500 \\ N & 150 \\ N & 150 \\ N & 200 \\ N & 70 \\ N & 100\end{array}$

N

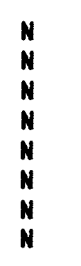

20
20
20
50
10
50
20
20

10
$<10$
$<10$
$N$
$N$
$N$
$N$
$N$

$N$
$N$
$N$
$N$
$N$
$N$
$N$

7
5
$<5$
$<5$
$<5$
5
$<5$
$<5$

HM0231S
HM0232S
HM0233S
HM0234S
HM0235S
HM0236S
HM0237S
HM0238S

Sn ppm-SQS Sr ppm-SQS Th ppm-SQS V ppm-SQS

$\begin{array}{cr}W \text { ppm-sQS } & Y \text { ppm-sas } \\ N & 10 \\ N & <10 \\ N & <10 \\ N & <10 \\ N & <10 \\ N & <10 \\ N & N \\ N & N\end{array}$

70
70
50
100
50
100
50
70

Zn ppm-SQS Zr ppm-SQS

Se ppm-MAS Te ppm-MAS

Sample Tl ppm-MAS AU ppm-MAS Hg ppm-AAS W ppm-VS F \%-SIE Pt ppb-ICP Pd ppb-ICP Rh ppb-ICP RU ppb-ICP Ir ppb-ICP

\begin{tabular}{|c|c|c|c|c|c|c|c|c|c|c|}
\hline $\begin{array}{l}\text { HMO231S } \\
\text { HM0232S } \\
H M 0233 S \\
H M 0234 S \\
H M 0235 S \\
H M 0236 S \\
H M 0237 S \\
H M 0238 S\end{array}$ & $\begin{array}{l}.4 \\
.2 \\
.2 \\
.3 \\
.3 \\
.3 \\
.4 \\
.25\end{array}$ & $\begin{array}{l}N \\
N \\
N \\
N \\
N \\
N \\
N \\
N\end{array}$ & $\begin{array}{l}.09 \\
.04 \\
.07 \\
.04 \\
.19 \\
.18 \\
.11 \\
10\end{array}$ & $\begin{array}{l}1 \\
1 \\
1 \\
2 \\
2 \\
2 \\
2\end{array}$ & $\begin{array}{l}.05 \\
.04 \\
.04 \\
.04 \\
.04 \\
.04 \\
.05 \\
.04\end{array}$ & $\begin{array}{l}<1 \\
<.5 \\
<.5 \\
4 \\
<1 \\
<1 \\
<1\end{array}$ & $\begin{array}{r}<.5 \\
.5 \\
<.5 \\
.5 \\
<.5 \\
.5 \\
1.2 \\
<.5\end{array}$ & $\begin{array}{l}<.5 \\
<.5 \\
<.5 \\
<.5 \\
<.5 \\
<.5 \\
<.5 \\
<.5\end{array}$ & $\begin{array}{l}<.5 \\
<.5 \\
<.5 \\
.6 \\
<.5 \\
.5 \\
<.5\end{array}$ & $\begin{array}{l}<.5 \\
<.5 \\
<.5 \\
<.5 \\
<.5 \\
<.5 \\
<.5 \\
<.5\end{array}$ \\
\hline
\end{tabular}


Table 4. Analytical data for heavy-mineral-concentrate samples collected from the Horn Mountains area, sleetmute quadrangle, Alaska. [SQS, semiquantitative emission spectrography; $N$, not detected; <, detected but below the limit of determination shown; $>$, determined to be greater than the value shown]

\begin{tabular}{|c|c|c|c|c|c|c|c|c|c|c|}
\hline Sample & Latitude & Longi tude & Ca \%-sas & Fe \%-SQS & Mg \%-SQS & Na $\%$-SQS & P \%-SQS & Ti $\%$-SQS & Ag ppm-SQS & As ppm-SQS \\
\hline $\begin{array}{l}\text { HM0001C } \\
\text { HMO002C } \\
\text { HM0003C } \\
\text { HM0004C } \\
\text { HM0005C } \\
\text { HM0006C } \\
\text { HM0007C } \\
\text { HM0008C } \\
\text { HM0009C } \\
\text { HM0010C }\end{array}$ & $\begin{array}{rrr}61 & 43 & 55 \\
61 & 42 & 59 \\
61 & 42 & 1 \\
61 & 39 & 48 \\
61 & 38 & 54 \\
61 & 38 & 9 \\
61 & 38 & 8 \\
61 & 36 & 35 \\
61 & 37 & 1 \\
61 & 41 & 25\end{array}$ & $\begin{array}{rrr}158 & 29 & 30 \\
158 & 29 & 58 \\
158 & 30 & 32 \\
158 & 34 & 5 \\
158 & 35 & 38 \\
158 & 31 & 45 \\
158 & 31 & 32 \\
158 & 24 & 37 \\
158 & 24 & 0 \\
158 & 26 & 26\end{array}$ & $\begin{array}{l}5 \\
5 \\
5 \\
5 \\
5 \\
2 \\
5 \\
1.5 \\
2 \\
7\end{array}$ & $\begin{array}{l}<.1 \\
<.1 \\
.15 \\
<.1 \\
.1 \\
.1 \\
.1 \\
<.1 \\
.1\end{array}$ & $\begin{array}{l}.07 \\
.07 \\
.1 \\
.1 \\
.05 \\
<.05 \\
.07 \\
.15 \\
.1 \\
.05\end{array}$ & $\begin{array}{l}N \\
N \\
N \\
N \\
N \\
N \\
N \\
N \\
N \\
N\end{array}$ & $\begin{array}{l}5 \\
5 \\
5 \\
3 \\
5 \\
5 \\
5 \\
.7 \\
2 \\
7\end{array}$ & $\begin{array}{r}>2 \\
>2 \\
>2 \\
>2 \\
>2 \\
>2 \\
>2 \\
>2 \\
>2 \\
2\end{array}$ & $\begin{array}{c}N \\
N \\
N \\
3 \\
15 \\
N \\
N \\
N \\
N \\
N\end{array}$ & $\begin{array}{l}N \\
N \\
N \\
N \\
N \\
N \\
N \\
N \\
N \\
N\end{array}$ \\
\hline $\begin{array}{l}\text { HM0011C } \\
\text { HM0012C } \\
\text { HM0013C } \\
\text { HM0014C } \\
\text { HM0015C } \\
\text { HM0016C } \\
\text { HM0017C } \\
\text { HM0018C } \\
\text { HM0019C } \\
\text { HM0020C }\end{array}$ & $\begin{array}{lll}61 & 41 & 22 \\
61 & 42 & 20 \\
61 & 44 & 0 \\
61 & 45 & 46 \\
61 & 45 & 44 \\
61 & 47 & 13 \\
61 & 47 & 43 \\
61 & 41 & 27 \\
61 & 44 & 27 \\
61 & 45 & 21\end{array}$ & $\begin{array}{lll}158 & 16 & 41 \\
158 & 24 & 35 \\
158 & 18 & 56 \\
158 & 20 & 17 \\
158 & 20 & 16 \\
158 & 24 & 15 \\
158 & 25 & 33 \\
158 & 36 & 46 \\
158 & 35 & 51 \\
158 & 36 & 40\end{array}$ & $\begin{array}{l}. .5 \\
1.5 \\
. .3 \\
5 \\
5 \\
1.5 \\
2 \\
5 \\
.7\end{array}$ & $\begin{array}{l}<.1 \\
.1 \\
.1 \\
<.1 \\
.1 \\
.2 \\
<.1 \\
N \\
<.1 \\
<.1\end{array}$ & $\begin{array}{l}.15 \\
.07 \\
.1 \\
.07 \\
.1 \\
.1 \\
.1 \\
.05 \\
.07 \\
.05\end{array}$ & $\begin{array}{l}.5 \\
.5 \\
.5 \\
N \\
N \\
N \\
<.5 \\
N \\
N\end{array}$ & $\begin{array}{l}2^{.5} \\
3^{.5} \\
7 \\
5 \\
5 \\
1.5 \\
5 \\
2\end{array}$ & $\begin{array}{r}2 \\
>2 \\
2 \\
>2 \\
>2 \\
>2 \\
>2 \\
2 \\
>2 \\
>2\end{array}$ & $\begin{array}{l}N \\
N \\
N \\
N \\
N \\
N \\
N \\
N \\
N \\
N\end{array}$ & $\begin{array}{l}N \\
N \\
N \\
N \\
N \\
N \\
N \\
N \\
N \\
N\end{array}$ \\
\hline $\begin{array}{l}\text {HMO021C } \\
\text { HM0022C } \\
\text { HM0023C } \\
\text { HM0024C } \\
\text { HM0025C } \\
\text { HM0026C } \\
\text { HM0027C } \\
\text { HM0028C } \\
\text { HM0029C } \\
\text { HM0030C }\end{array}$ & $\begin{array}{rrr}61 & 46 & 13 \\
61 & 46 & 9 \\
61 & 49 & 39 \\
61 & 46 & 57 \\
61 & 51 & 31 \\
61 & 49 & 10 \\
61 & 53 & 12 \\
61 & 54 & 7 \\
61 & 53 & 53 \\
61 & 56 & 11\end{array}$ & $\begin{array}{rrr}158 & 33 & 24 \\
158 & 33 & 30 \\
158 & 26 & 24 \\
158 & 21 & 45 \\
158 & 18 & 5 \\
158 & 30 & 40 \\
158 & 28 & 40 \\
158 & 22 & 5 \\
158 & 16 & 37 \\
158 & 14 & 31\end{array}$ & $\begin{array}{l}5 \\
3 \\
2 \\
2 \\
2 \\
5 \\
7 \\
5 \\
2 \\
2\end{array}$ & $\begin{array}{l}.1 \\
.1 \\
.1 \\
N \\
.1 \\
.1 \\
.1 \\
. .\end{array}$ & $\begin{array}{l}.1 \\
.07 \\
.1 \\
.07 \\
.1 \\
.07 \\
.15 \\
<.05 \\
-- \\
.07\end{array}$ & $\begin{array}{l}<.5 \\
N \\
<.5 \\
N \\
<.5 \\
N \\
N \\
N \\
N \\
N\end{array}$ & $\begin{array}{l}3 \\
5 \\
3 \\
5 \\
2 \\
5 \\
5 \\
3 \\
5\end{array}$ & $\begin{array}{r}2 \\
>2 \\
>2 \\
>2 \\
>2 \\
>2 \\
2 \\
1 \\
2 \\
2\end{array}$ & $\begin{array}{c}N \\
20 \\
N \\
N \\
N \\
N \\
N \\
N \\
-\therefore \\
N\end{array}$ & \begin{tabular}{c}
$N$ \\
$N$ \\
$N$ \\
$N$ \\
$N$ \\
$N$ \\
$N$ \\
$N$ \\
\hdashline \\
$N$
\end{tabular} \\
\hline $\begin{array}{l}\text { HMO031C } \\
\text { HM0032C } \\
\text { HM0033C } \\
\text { HM0034C } \\
\text { HM0035C } \\
\text { HM0036C } \\
\text { HM0037C } \\
\text { HM0038C } \\
\text { HM0039C } \\
\text { HM0040C }\end{array}$ & $\begin{array}{lll}61 & 50 & 56 \\
61 & 48 & 47 \\
61 & 47 & 21 \\
61 & 45 & 57 \\
61 & 55 & 49 \\
61 & 55 & 48 \\
61 & 59 & 19 \\
61 & 57 & 15 \\
61 & 59 & 16 \\
61 & 55 & 36\end{array}$ & $\begin{array}{rrr}158 & 11 & 41 \\
158 & 7 & 21 \\
158 & 15 & 17 \\
158 & 15 & 48 \\
158 & 50 & 5 \\
158 & 58 & 38 \\
158 & 47 & 3 \\
158 & 40 & 8 \\
158 & 34 & 58 \\
158 & 36 & 1\end{array}$ & $\begin{array}{l}1.5 \\
.5 \\
.5 \\
.2 \\
.3 \\
N \\
1.5 \\
2.5 \\
.5 \\
.2\end{array}$ & $\begin{array}{l}N \\
<.1 \\
.1 \\
N \\
<.1 \\
N \\
-1 \\
<.1 \\
N \\
N\end{array}$ & $\begin{array}{l}.1 \\
.07 \\
.15 \\
.05 \\
.05 \\
<.05 \\
.05 \\
.1 \\
.05 \\
<.05\end{array}$ & $\begin{array}{l}N \\
N \\
<.5 \\
N \\
N \\
N \\
N \\
N \\
N \\
N\end{array}$ & $\begin{array}{l}<.5 \\
<.5 \\
.5 \\
.7 \\
1 \\
<.5 \\
2 \\
3 \\
.7\end{array}$ & $\begin{array}{r}>2 \\
2 \\
2 \\
2 \\
>2 \\
>2 \\
>2 \\
>2 \\
>2 \\
>2\end{array}$ & $\begin{array}{l}N \\
N \\
N \\
N \\
N \\
N \\
N \\
N \\
N \\
N\end{array}$ & $\begin{array}{l}N \\
N \\
N \\
N \\
N \\
N \\
N \\
N \\
N \\
N\end{array}$ \\
\hline $\begin{array}{l}\text { HM0041C } \\
\text { HM0042C } \\
\text { HM0043C } \\
\text { HM0044C } \\
\text { HM0045C } \\
\text { HM0046C } \\
\text { HM0047C } \\
\text { HM0048C } \\
\text { HM0049C } \\
\text { HM0050C }\end{array}$ & $\begin{array}{rrr}61 & 59 & 0 \\
61 & 51 & 25 \\
61 & 53 & 42 \\
61 & 51 & 52 \\
61 & 47 & 45 \\
61 & 45 & 3 \\
61 & 51 & 40 \\
61 & 43 & 44 \\
61 & 39 & 55 \\
61 & 40 & 6\end{array}$ & $\begin{array}{rrr}158 & 18 & 44 \\
158 & 38 & 19 \\
158 & 55 & 25 \\
158 & 45 & 54 \\
158 & 50 & 32 \\
158 & 55 & 1 \\
158 & 59 & 9 \\
158 & 59 & 17 \\
158 & 48 & 41 \\
158 & 45 & 55\end{array}$ & $\begin{array}{l}.7 \\
1 \\
.3 \\
.7 \\
.3 \\
1.3 \\
.5 \\
<.1\end{array}$ & $\begin{array}{l}N \\
<.1 \\
<.1 \\
<.1 \\
N \\
N \\
<.1 \\
\dot{N} \\
N\end{array}$ & $\begin{array}{l}<.05 \\
.07 \\
.1 \\
.07 \\
.05 \\
<.05 \\
.07 \\
.07 \\
.05 \\
.\end{array}$ & $\begin{array}{l}N \\
N \\
N \\
N \\
N \\
N \\
N \\
N \\
N \\
N\end{array}$ & $\begin{array}{l}1.5 \\
3 \\
1.5 \\
.7 \\
1.5 \\
1 \\
2 \\
.7 \\
1.5\end{array}$ & $\begin{array}{l}>2 \\
>2 \\
>2 \\
>2 \\
>2 \\
>2 \\
>2 \\
>2 \\
>2 \\
>2\end{array}$ & $\begin{array}{l}N \\
N \\
N \\
N \\
N \\
N \\
N \\
N \\
N \\
N\end{array}$ & $\begin{array}{l}N \\
N \\
N \\
N \\
N \\
N \\
N \\
N \\
N \\
N\end{array}$ \\
\hline $\begin{array}{l}\text { HM0100C } \\
\text { HM0101C } \\
\text { HM0102C } \\
\text { HM0103C } \\
\text { HM0104C } \\
\text { HM0105C } \\
\text { HM0106C } \\
\text { HM0107C } \\
\text { HM0108C } \\
\text { HM0109C }\end{array}$ & $\begin{array}{rrr}61 & 43 & 56 \\
61 & 44 & 18 \\
61 & 41 & 7 \\
61 & 38 & 19 \\
61 & 37 & 24 \\
61 & 36 & 49 \\
61 & 38 & 11 \\
61 & 41 & 6 \\
61 & 40 & 4 \\
61 & 43 & 6\end{array}$ & $\begin{array}{rrr}158 & 29 & 19 \\
158 & 28 & 15 \\
158 & 31 & 18 \\
158 & 34 & 12 \\
158 & 29 & 28 \\
158 & 28 & 30 \\
158 & 25 & 7 \\
158 & 28 & 10 \\
158 & 19 & 13 \\
158 & 23 & 27\end{array}$ & $\begin{array}{l}2 \\
2 \\
5 \\
3 \\
5 \\
.2 \\
.3 \\
1^{.3} \\
.3\end{array}$ & $\begin{array}{l}.1 \\
<.1 \\
.15 \\
<.1 \\
<.1 \\
N \\
N \\
<.1 \\
N \\
N\end{array}$ & $\begin{array}{l}.1 \\
.07 \\
.1 \\
.05 \\
.07 \\
.07 \\
.05 \\
.05 \\
.05 \\
<.05\end{array}$ & $\begin{array}{l}N \\
N \\
N \\
N \\
N \\
N \\
N \\
N \\
N \\
N\end{array}$ & $\begin{array}{l}3 \\
3 \\
3 \\
3 \\
5 \\
1 \\
3^{.5} \\
3^{<.5}\end{array}$ & $\begin{array}{l}>2 \\
>2 \\
>2 \\
2 \\
>2 \\
>2 \\
>2 \\
>2 \\
>2 \\
>2\end{array}$ & $\begin{array}{l}N \\
N \\
N \\
N \\
N \\
N \\
N \\
N \\
N \\
N\end{array}$ & $\begin{array}{l}N \\
N \\
N \\
N \\
N \\
N \\
N \\
N \\
N \\
N\end{array}$ \\
\hline $\begin{array}{l}\text { HM0110C } \\
\text { HM0111C } \\
\text { HM0112C } \\
\text { HM0113C } \\
\text { HM0114C }\end{array}$ & $\begin{array}{llr}61 & 43 & 1 \\
61 & 44 & 55 \\
61 & 45 & 59 \\
61 & 48 & 35 \\
61 & 39 & 56\end{array}$ & $\begin{array}{rrr}158 & 17 & 45 \\
158 & 21 & 58 \\
158 & 25 & 43 \\
158 & 19 & 4 \\
158 & 36 & 13\end{array}$ & $\begin{array}{l}1 \\
5 \\
5 \\
1.5 \\
7\end{array}$ & $\begin{array}{l}.15 \\
.1 \\
.2 \\
<.1 \\
<.1\end{array}$ & $\begin{array}{l}.1 \\
.1 \\
.1 \\
.05 \\
.07\end{array}$ & $\begin{array}{l}N \\
N \\
N \\
N \\
N\end{array}$ & $\begin{array}{l}2 \\
5 \\
5 \\
1.5 \\
7\end{array}$ & $\begin{array}{l}>2 \\
>2 \\
>2 \\
>2 \\
>2\end{array}$ & $\begin{array}{l}N \\
N \\
N \\
N \\
N\end{array}$ & $\begin{array}{l}\mathbf{N} \\
\mathbf{N} \\
\mathbf{N} \\
\mathbf{N} \\
\mathbf{N}\end{array}$ \\
\hline
\end{tabular}


Table 4. Analytical data for heavy-mineral-concentrate samples collected from the Horn Mountains area--Continued.

\begin{tabular}{|c|c|c|c|c|c|c|c|c|c|}
\hline Sample & Au ppm-SQS & B ppm-SQS & Ba ppm-SQS & Be ppm-SQS & Bi ppm-SQS & Cd ppm-Sas & Co ppm-Sas & Cr ppon-sas & Cu ppm-sQS \\
\hline $\begin{array}{l}\text { HM0001C } \\
H M 0002 C \\
H M 0003 C \\
H M 0004 C \\
H M 0005 C \\
H M 0006 C \\
H M 0007 C \\
H M 0008 C \\
H M 0009 C \\
H M 0010 C\end{array}$ & $\begin{array}{r}N \\
<20 \\
N \\
<20 \\
20 \\
N \\
N \\
N \\
N \\
N\end{array}$ & $\begin{array}{r}50 \\
50 \\
150 \\
20 \\
30 \\
20 \\
30 \\
20 \\
<20 \\
N\end{array}$ & $\begin{array}{r}100 \\
200 \\
150 \\
7,000 \\
200 \\
1,000 \\
1,000 \\
500 \\
300 \\
70\end{array}$ & $\begin{array}{l}N \\
N \\
N \\
N \\
N \\
N \\
N \\
7 \\
N \\
N\end{array}$ & $\begin{array}{r}N \\
300 \\
N \\
200 \\
70 \\
N \\
N \\
N \\
N \\
N\end{array}$ & $\begin{array}{l}N \\
N \\
N \\
N \\
N \\
N \\
N \\
N \\
N \\
N\end{array}$ & $\begin{array}{r}N \\
N \\
N \\
<20 \\
N \\
N \\
N \\
N \\
N \\
N\end{array}$ & $\begin{array}{r}500 \\
500 \\
700 \\
200 \\
70 \\
500 \\
300 \\
100 \\
150 \\
70\end{array}$ & $\begin{array}{l}N \\
N \\
N \\
N \\
N \\
N \\
N \\
N \\
N \\
N\end{array}$ \\
\hline $\begin{array}{l}\text { HM0011C } \\
\text { HM0012C } \\
H M 0013 C \\
\text { HM014C } \\
\text { HM0015C } \\
\text { HM0016C } \\
\text { HM0017C } \\
\text { HM0018C } \\
\text { HM0019C } \\
\text { HM0020C }\end{array}$ & $\begin{array}{l}N \\
N \\
N \\
N \\
N \\
N \\
N \\
N \\
N \\
N\end{array}$ & $\begin{array}{r}N \\
N \\
<20 \\
N \\
50 \\
30 \\
30 \\
N \\
<20 \\
N\end{array}$ & $\begin{array}{r}500 \\
200 \\
1,000 \\
150 \\
200 \\
200 \\
150 \\
100 \\
100 \\
100\end{array}$ & $\begin{array}{l}N \\
N \\
N \\
N \\
N \\
N \\
N \\
N \\
N \\
N\end{array}$ & $\begin{array}{r}N \\
N \\
N \\
N \\
N \\
100 \\
30 \\
N \\
N \\
N\end{array}$ & $\begin{array}{l}N \\
N \\
N \\
N \\
N \\
N \\
N \\
N \\
N \\
N\end{array}$ & $\begin{array}{l}N \\
N \\
N \\
N \\
N \\
N \\
N \\
N \\
N \\
N\end{array}$ & $\begin{array}{r}30 \\
700 \\
50 \\
700 \\
700 \\
1,000 \\
1,500 \\
50 \\
500 \\
1,500\end{array}$ & $\begin{array}{l}N \\
N \\
N \\
N \\
N \\
N \\
N \\
N \\
N \\
N\end{array}$ \\
\hline $\begin{array}{l}\text { HM0021C } \\
\text { HM0022C } \\
H M 0023 C \\
\text { HM0024C } \\
\text { HM0025C } \\
\text { HM0026C } \\
\text { HM0027C } \\
\text { HM0028C } \\
\text { HM0029C } \\
\text { HM0030C }\end{array}$ & $\begin{array}{r}N \\
500 \\
N \\
N \\
N \\
N \\
N \\
N \\
- \\
N\end{array}$ & $\begin{array}{r}N \\
100 \\
50 \\
N \\
20 \\
N \\
N \\
N \\
-- \\
<20\end{array}$ & $\begin{array}{l}200 \\
150 \\
300 \\
200 \\
300 \\
100 \\
300 \\
100 \\
-- \\
500\end{array}$ & $\begin{array}{l}N \\
N \\
N \\
N \\
N \\
N \\
N \\
N \\
-N \\
N\end{array}$ & $\begin{array}{c}N \\
N \\
50 \\
N \\
N \\
N \\
N \\
N \\
-N \\
N\end{array}$ & $\begin{array}{l}N \\
N \\
N \\
N \\
N \\
N \\
N \\
N \\
-- \\
N\end{array}$ & $\begin{array}{c}N \\
N \\
N \\
N \\
N \\
N \\
N \\
N \\
-N \\
N\end{array}$ & $\begin{array}{r}150 \\
700 \\
2,000 \\
1,000 \\
50 \\
300 \\
70 \\
30 \\
-- \\
50\end{array}$ & $\begin{array}{c}N \\
N \\
N \\
N \\
N \\
N \\
N \\
N \\
-N \\
N\end{array}$ \\
\hline $\begin{array}{l}\text { HM0031C } \\
\text { HM0032C } \\
\text { HM0033C } \\
\text { HM0034C } \\
\text { HM0035C } \\
\text { HM0036C } \\
\text { HM0037C } \\
\text { HM0038C } \\
\text { HM0039C } \\
\text { HM0040C }\end{array}$ & $\begin{array}{l}N \\
N \\
N \\
N \\
N \\
N \\
N \\
N \\
N \\
N\end{array}$ & $\begin{array}{r}N \\
N \\
<20 \\
<20 \\
N \\
<20 \\
20 \\
20 \\
N \\
<20\end{array}$ & $\begin{array}{r}300 \\
500 \\
500 \\
500 \\
700 \\
300 \\
500 \\
200 \\
200 \\
3,000\end{array}$ & $\begin{array}{l}N \\
N \\
N \\
N \\
N \\
N \\
N \\
N \\
N \\
N\end{array}$ & $\begin{array}{l}N \\
N \\
N \\
N \\
N \\
N \\
N \\
N \\
N \\
N\end{array}$ & $\begin{array}{l}N \\
N \\
N \\
N \\
N \\
N \\
N \\
N \\
N \\
N\end{array}$ & $\begin{array}{l}N \\
N \\
N \\
N \\
N \\
N \\
N \\
N \\
N \\
N\end{array}$ & $\begin{array}{r}50 \\
50 \\
50 \\
70 \\
1,000 \\
300 \\
150 \\
50 \\
70 \\
100\end{array}$ & $\begin{array}{l}N \\
N \\
N \\
N \\
N \\
N \\
N \\
N \\
N \\
N\end{array}$ \\
\hline $\begin{array}{l}\text { HM0041C } \\
\text { HM0042C } \\
\text { HM0043C } \\
\text { HM0044C } \\
\text { HM0045C } \\
\text { HM0046C } \\
\text { HM0047C } \\
\text { HM0048C } \\
\text { HM0049C } \\
\text { HM0050C }\end{array}$ & $\begin{array}{l}N \\
N \\
N \\
N \\
N \\
N \\
N \\
N \\
N \\
N\end{array}$ & $\begin{array}{r}N \\
20 \\
20 \\
20 \\
N \\
<20 \\
20 \\
70 \\
20 \\
N\end{array}$ & $\begin{array}{r}500 \\
500 \\
1,000 \\
300 \\
300 \\
1,500 \\
2,000 \\
500 \\
500 \\
100\end{array}$ & $\begin{array}{l}N \\
N \\
N \\
N \\
N \\
N \\
N \\
N \\
N \\
N\end{array}$ & $\begin{array}{l}N \\
N \\
N \\
N \\
N \\
N \\
N \\
N \\
N \\
N\end{array}$ & $\begin{array}{l}N \\
N \\
N \\
N \\
N \\
N \\
N \\
N \\
N \\
N\end{array}$ & $\begin{array}{l}N \\
N \\
N \\
N \\
N \\
N \\
N \\
N \\
N \\
N\end{array}$ & $\begin{array}{r}20 \\
70 \\
100 \\
200 \\
20 \\
500 \\
500 \\
300 \\
300 \\
70\end{array}$ & $\begin{array}{l}N \\
N \\
N \\
N \\
N \\
N \\
N \\
N \\
N \\
N\end{array}$ \\
\hline $\begin{array}{l}\text { HM0100C } \\
\text { HM0101C } \\
\text { HM0102C } \\
\text { HM0103C } \\
\text { HM0104C } \\
\text { HM0105C } \\
\text { HM0106C } \\
\text { HM0107C } \\
\text { HM0108C } \\
\text { HM0109C }\end{array}$ & $\begin{array}{l}30 \\
N \\
N \\
N \\
N \\
N \\
N \\
N \\
N \\
N\end{array}$ & $\begin{array}{r}30 \\
30 \\
20 \\
N \\
<20 \\
30 \\
<20 \\
N \\
<20 \\
N\end{array}$ & $\begin{array}{r}150 \\
100 \\
70 \\
300 \\
5,000 \\
700 \\
500 \\
150 \\
500 \\
100\end{array}$ & $\begin{array}{l}<2 \\
N \\
N \\
N \\
N \\
N \\
N \\
N \\
N \\
N\end{array}$ & $\begin{array}{r}500 \\
1,000 \\
N \\
N \\
N \\
N \\
N \\
N \\
N \\
N\end{array}$ & $\begin{array}{l}N \\
N \\
N \\
N \\
N \\
N \\
N \\
N \\
N \\
N\end{array}$ & $\begin{array}{l}N \\
N \\
N \\
N \\
N \\
N \\
N \\
N \\
N \\
N\end{array}$ & $\begin{array}{r}700 \\
1,000 \\
500 \\
500 \\
30 \\
70 \\
30 \\
700 \\
50 \\
1,000\end{array}$ & $\begin{array}{l}N \\
N \\
N \\
N \\
N \\
N \\
N \\
N \\
N \\
N\end{array}$ \\
\hline $\begin{array}{l}\text { HM0110C } \\
\text { HM0111C } \\
\text { HM0112C } \\
\text { HM0113C } \\
\text { HM0114C }\end{array}$ & $\begin{array}{l}N \\
N \\
N \\
N \\
N\end{array}$ & $\begin{array}{r}50 \\
50 \\
50 \\
<20 \\
<20\end{array}$ & $\begin{array}{r}10,000 \\
150 \\
200 \\
300 \\
200\end{array}$ & $\begin{array}{l}N \\
N \\
N \\
N \\
N\end{array}$ & 1,000 & $\begin{array}{l}N \\
N \\
N \\
N \\
N\end{array}$ & $\begin{array}{l}N \\
N \\
N \\
N \\
N\end{array}$ & $\begin{array}{r}200 \\
500 \\
1,000 \\
100 \\
150\end{array}$ & $\begin{array}{l}N \\
N \\
N \\
N \\
N\end{array}$ \\
\hline
\end{tabular}


Table 4. Analytical data for heavy-mineral-concentrate samples collected from the Horn Mountains area--Continued.

Sample Ga ppm-SQS Ge ppm-SQS La ppm-SQS Mn ppm-SQS Mo ppm-SQS Nb ppm-SOS Ni ppm-SOS Pb ppm-SQS Sb ppm-SQS SC ppm-SOS

\begin{tabular}{|c|c|c|c|c|c|c|c|c|c|c|}
\hline $\begin{array}{l}\text { HMO001C } \\
\text { HMO002C } \\
\text { HM0003C } \\
\text { HM0004C } \\
\text { HM0005C } \\
\text { HM0006C } \\
\text { HM0007C } \\
\text { HM0008C } \\
\text { HM0009C } \\
\text { HM0010C }\end{array}$ & $\begin{array}{r}N \\
N \\
<10 \\
N \\
N \\
N \\
N \\
10 \\
15 \\
N\end{array}$ & $\begin{array}{l}N \\
N \\
N \\
N \\
N \\
N \\
N \\
N \\
N \\
N\end{array}$ & $\begin{array}{l}150 \\
150 \\
100 \\
150 \\
100 \\
150 \\
150 \\
<100 \\
<100 \\
200\end{array}$ & $\begin{array}{l}200 \\
200 \\
300 \\
100 \\
100 \\
100 \\
150 \\
30 \\
50 \\
50\end{array}$ & $\begin{array}{l}N \\
N \\
N \\
N \\
N \\
N \\
N \\
N \\
N \\
N\end{array}$ & $\begin{array}{r}100 \\
<50 \\
<50 \\
50 \\
<50 \\
50 \\
70 \\
<50 \\
N \\
N\end{array}$ & $\begin{array}{c}N \\
N \\
N \\
10 \\
N \\
N \\
N \\
N \\
N \\
N\end{array}$ & $\begin{array}{r}N \\
200 \\
20 \\
20 \\
N \\
N \\
N \\
N \\
N \\
N \\
N \\
N\end{array}$ & 3 & $\begin{array}{r}15 \\
10 \\
<10 \\
10 \\
<10 \\
30 \\
20 \\
20 \\
<10 \\
<10\end{array}$ \\
\hline $\begin{array}{l}\text { HMO011C } \\
\text { HM0012C } \\
\text { HM0013C } \\
\text { HM0014C } \\
\text { HM0015C } \\
\text { HM0016C } \\
\text { HM0017C } \\
\text { HM0018C } \\
\text { HM0019C } \\
\text { HM0020C }\end{array}$ & $\begin{array}{r}<10 \\
\mathbf{N} \\
<10 \\
\mathbf{N} \\
\mathbf{N} \\
\mathbf{N} \\
\mathbf{N} \\
\mathbf{N} \\
\mathbf{N} \\
\mathbf{N}\end{array}$ & $\begin{array}{l}N \\
N \\
N \\
N \\
N \\
N \\
N \\
N \\
N \\
N\end{array}$ & $\begin{array}{r}N \\
N \\
N \\
N \\
150 \\
150 \\
<100 \\
N \\
100 \\
N\end{array}$ & $\begin{array}{r}20 \\
20 \\
<20 \\
30 \\
50 \\
200 \\
50 \\
20 \\
50 \\
20\end{array}$ & $\begin{array}{l}N \\
N \\
N \\
N \\
N \\
N \\
N \\
N \\
N \\
N\end{array}$ & $\begin{array}{r}N \\
70 \\
N \\
<50 \\
50 \\
50 \\
50 \\
N \\
<50 \\
70\end{array}$ & $\begin{array}{l}N \\
N \\
N \\
N \\
N \\
N \\
N \\
N \\
N \\
N\end{array}$ & $\begin{array}{r}N \\
20 \\
N \\
N \\
N \\
N \\
N \\
N \\
N \\
<20\end{array}$ & 1 & $\begin{array}{r}<10 \\
30 \\
<10 \\
30 \\
70 \\
20 \\
30 \\
N \\
30 \\
100\end{array}$ \\
\hline $\begin{array}{l}\text { HMO021C } \\
\text { HM0022C } \\
\text { HM0023C } \\
\text { HM0024C } \\
\text { HMO025C } \\
\text { HM0026C } \\
\text { HM0027C } \\
\text { HM0028C } \\
\text { HM0029C } \\
\text { HM003OC }\end{array}$ & $\begin{array}{c}\mathbf{N} \\
\mathbf{N} \\
\mathbf{N} \\
\mathbf{N} \\
\mathbf{N} \\
\mathbf{N} \\
\mathbf{N} \\
\mathbf{N} \\
-\mathbf{N}\end{array}$ & $\begin{array}{c}N \\
N \\
N \\
N \\
N \\
N \\
N \\
N \\
-1 \\
N\end{array}$ & $\begin{array}{r}100 \\
100 \\
<100 \\
<100 \\
<100 \\
150 \\
300 \\
200 \\
-- \\
100\end{array}$ & $\begin{array}{r}50 \\
70 \\
70 \\
30 \\
50 \\
100 \\
150 \\
200 \\
-- \\
30\end{array}$ & \begin{tabular}{c}
$N$ \\
$N$ \\
$N$ \\
$N$ \\
$N$ \\
$N$ \\
$N$ \\
$N$ \\
\hdashline- \\
$N$
\end{tabular} & $\begin{array}{r}N \\
<50 \\
70 \\
<50 \\
<50 \\
N \\
N \\
N \\
-- \\
N\end{array}$ & $\begin{array}{l}N \\
N \\
N \\
N \\
N \\
N \\
N \\
N \\
-\therefore \\
N\end{array}$ & $\begin{array}{r}N \\
<20 \\
20 \\
N \\
<20 \\
N \\
70 \\
N \\
-- \\
<20\end{array}$ & $\begin{array}{c}1 \\
1 \\
1 \\
1 \\
1 \\
1 \\
1 \\
-1\end{array}$ & $\begin{array}{r}<10 \\
30 \\
15 \\
30 \\
10 \\
<10 \\
15 \\
20 \\
-- \\
<10\end{array}$ \\
\hline $\begin{array}{l}\text { HMO031C } \\
\text { HMO032C } \\
\text { HM0033C } \\
\text { HM0034C } \\
\text { HM0035C } \\
\text { HM0036C } \\
\text { HM0037C } \\
\text { HM0038C } \\
\text { HM0039C } \\
\text { HM0040C }\end{array}$ & $\begin{array}{r}\mathbf{N} \\
10 \\
<10 \\
<10 \\
\mathbf{N} \\
\mathbf{N} \\
\mathbf{N} \\
\mathbf{N} \\
\mathbf{N} \\
\mathbf{N}\end{array}$ & $\begin{array}{l}N \\
N \\
N \\
N \\
N \\
N \\
N \\
N \\
N \\
N\end{array}$ & $\begin{array}{r}N \\
N \\
N \\
N \\
N \\
N \\
N \\
100 \\
N \\
100\end{array}$ & $\begin{array}{r}30 \\
30 \\
30 \\
<20 \\
<20 \\
N \\
20 \\
30 \\
<20 \\
<20\end{array}$ & $\begin{array}{l}N \\
N \\
N \\
N \\
N \\
N \\
N \\
N \\
N \\
N\end{array}$ & $\begin{array}{r}N \\
N \\
N \\
N \\
N \\
N \\
N \\
50 \\
<50 \\
<50\end{array}$ & $\begin{array}{l}N \\
N \\
N \\
N \\
N \\
N \\
N \\
N \\
N \\
N\end{array}$ & $\begin{array}{r}N \\
N \\
<20 \\
N \\
N \\
N \\
N \\
<20 \\
N \\
N\end{array}$ & 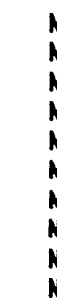 & $\begin{array}{r}15 \\
10 \\
<10 \\
10 \\
<10 \\
20 \\
30 \\
10 \\
15 \\
15\end{array}$ \\
\hline $\begin{array}{l}\text { HMOO41C } \\
\text { HM0042C } \\
\text { HM0043C } \\
\text { HM0044C } \\
\text { HM0045C } \\
\text { HM0046C } \\
\text { HM0047C } \\
\text { HM0048C } \\
\text { HM0049C } \\
\text { HM0050C }\end{array}$ & $\begin{array}{l}\mathbf{N} \\
\boldsymbol{N} \\
\mathbf{N} \\
\mathbf{N} \\
\mathbf{N} \\
\mathbf{N} \\
\mathbf{N} \\
\mathbf{N} \\
\mathbf{N} \\
\mathbf{N}\end{array}$ & $\begin{array}{l}N \\
N \\
N \\
N \\
N \\
N \\
N \\
N \\
N \\
N\end{array}$ & $\begin{array}{r}N \\
<100 \\
100 \\
<100 \\
N \\
<100 \\
100 \\
<100 \\
N \\
N\end{array}$ & $\begin{array}{r}<20 \\
50 \\
30 \\
N \\
<20 \\
<20 \\
20 \\
20 \\
<20 \\
N\end{array}$ & $\begin{array}{l}N \\
N \\
N \\
N \\
N \\
N \\
N \\
N \\
N \\
N\end{array}$ & $\begin{array}{r}N \\
N \\
<50 \\
50 \\
N \\
N \\
N \\
50 \\
<50 \\
N\end{array}$ & $\begin{array}{l}N \\
N \\
N \\
N \\
N \\
N \\
N \\
N \\
N \\
N\end{array}$ & $\begin{array}{r}N \\
<20 \\
N \\
<20 \\
<20 \\
N \\
<20 \\
N \\
N \\
N\end{array}$ & in & $\begin{array}{r}<10 \\
15 \\
20 \\
<10 \\
<10 \\
20 \\
<10 \\
<10 \\
10 \\
<10\end{array}$ \\
\hline $\begin{array}{l}\text { HM0100C } \\
\text { HM0101C } \\
\text { HM0102C } \\
\text { HM0103C } \\
\text { HM0104C } \\
\text { HM0105C } \\
\text { HM0106C } \\
\text { HM0107C } \\
\text { HM0108C } \\
\text { HM0109C }\end{array}$ & $\begin{array}{r}N \\
N \\
N \\
<10 \\
N \\
N \\
N \\
<10 \\
N \\
N \\
N\end{array}$ & $\begin{array}{l}N \\
N \\
N \\
N \\
N \\
N \\
N \\
N \\
N \\
N\end{array}$ & $\begin{array}{r}100 \\
<100 \\
200 \\
150 \\
150 \\
100 \\
N \\
N \\
N \\
N\end{array}$ & $\begin{array}{l}200 \\
100 \\
200 \\
100 \\
150 \\
<20 \\
<20 \\
<20 \\
<20 \\
<20\end{array}$ & $\begin{array}{l}N \\
N \\
N \\
N \\
N \\
N \\
N \\
N \\
N \\
N\end{array}$ & $\begin{array}{r}50 \\
50 \\
<50 \\
<50 \\
N \\
N 50 \\
N \\
N 50 \\
N \\
N \\
<50\end{array}$ & $\begin{array}{l}N \\
N \\
N \\
N \\
N \\
N \\
N \\
N \\
N \\
N\end{array}$ & $\begin{array}{r}<20 \\
20 \\
N \\
<20 \\
<20 \\
N \\
N \\
20 \\
N \\
30\end{array}$ & ? & $\begin{array}{r}10 \\
10 \\
20 \\
20 \\
N \\
15 \\
<10 \\
30 \\
10 \\
20\end{array}$ \\
\hline $\begin{array}{l}\text { HM0110C } \\
\text { HM0111C } \\
\text { HM0112C } \\
\text { HM0113C } \\
\text { HM0114C }\end{array}$ & $\begin{array}{l}N \\
N \\
N \\
N \\
N\end{array}$ & $\begin{array}{l}\mathbf{N} \\
\mathbf{N} \\
\mathbf{N} \\
\mathbf{N} \\
\mathbf{N}\end{array}$ & $\begin{array}{r}100 \\
150 \\
150 \\
<100 \\
200\end{array}$ & $\begin{array}{r}<20 \\
100 \\
100 \\
20 \\
100\end{array}$ & $\begin{array}{l}\mathbf{N} \\
\mathbf{N} \\
\mathbf{N} \\
\mathbf{N} \\
\mathbf{N}\end{array}$ & $\begin{array}{r}<50 \\
50 \\
70 \\
<50 \\
<50\end{array}$ & $\begin{array}{l}\mathbf{N} \\
\mathbf{N} \\
\mathbf{N} \\
\mathbf{N} \\
\mathbf{N}\end{array}$ & $\begin{array}{r}N \\
<20 \\
50 \\
N \\
<20\end{array}$ & N & $\begin{array}{l}30 \\
20 \\
20 \\
10 \\
10\end{array}$ \\
\hline
\end{tabular}


Table 4. Analytical data for heavy-mineral-concentrate samples collected from the Horn Mountains area--Continued.

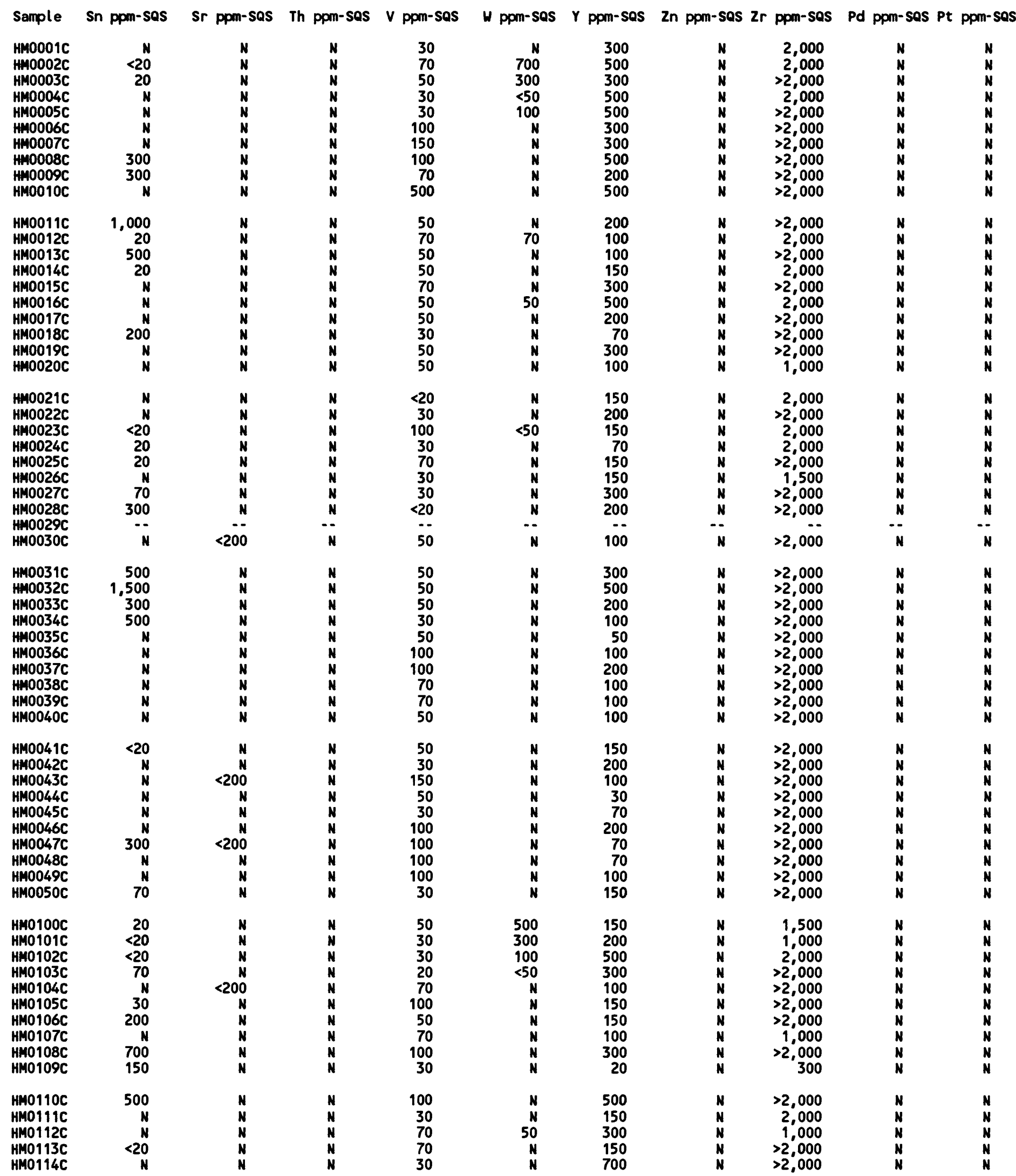


Table 4. Analytical data for heavy-mineral-concentrate samples collected from the Horn Mountains area--Continued.

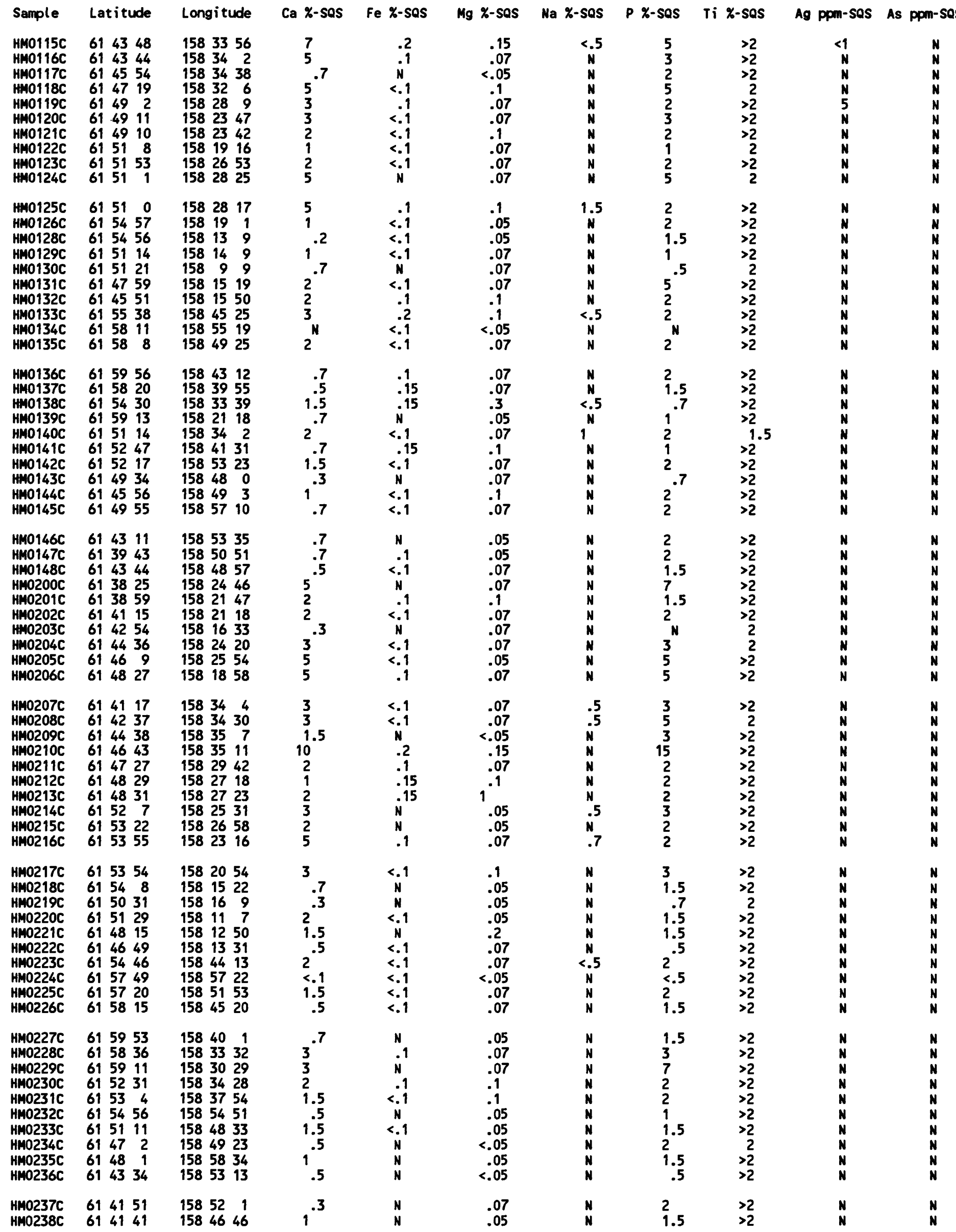


Table 4. Analytical data for heavy-mineral-concentrate samples collected from the Horn Mountains area--Continued.

\begin{tabular}{|c|c|c|c|c|c|c|c|c|c|}
\hline Sample & Au ppm-SQS & 8 ppm-sas & Ba ppm-Sas & Be ppm-sas & Bi ppm-Sas & Cd ppm-sas & Co ppm-Sas & Cr ppm-sas & Cu ppm-sas \\
\hline $\begin{array}{l}\text { HM0115C } \\
\text { HM0116C } \\
\text { HM0117C } \\
\text { HM0118C } \\
\text { HM0119C } \\
\text { HM0120C } \\
\text { HM0121C } \\
\text { HM0122C } \\
\text { HM0123C } \\
\text { HM0124C }\end{array}$ & $\begin{array}{l}N \\
N \\
N \\
N \\
30 \\
N \\
N \\
N \\
N \\
N\end{array}$ & $\begin{array}{r}50 \\
20 \\
N \\
N \\
30 \\
N \\
N \\
N \\
200 \\
20\end{array}$ & $\begin{array}{r}100 \\
70 \\
50 \\
200 \\
100 \\
100 \\
300 \\
300 \\
300 \\
500\end{array}$ & $\begin{array}{l}N \\
N \\
N \\
N \\
N \\
N \\
<2 \\
N \\
N \\
2\end{array}$ & $\begin{array}{r}N \\
500 \\
N \\
N \\
M \\
N \\
M \\
N \\
N \\
N\end{array}$ & $\begin{array}{l}N \\
N \\
N \\
N \\
N \\
N \\
N \\
N \\
N \\
N\end{array}$ & $\begin{array}{l}N \\
N \\
N \\
N \\
N \\
N \\
N \\
N \\
N \\
N\end{array}$ & $\begin{array}{r}1,000 \\
200 \\
500 \\
500 \\
1,000 \\
1,000 \\
1,500 \\
70 \\
1,500 \\
100\end{array}$ & $\begin{array}{r}<10 \\
N \\
N \\
N \\
N \\
N \\
N \\
N \\
N \\
N\end{array}$ \\
\hline $\begin{array}{l}\text { HM0125C } \\
\text { HM0126C } \\
\text { HM0128C } \\
\text { HM0129C } \\
\text { HM0130C } \\
\text { HM0131C } \\
\text { HM0132C } \\
\text { HM0133C } \\
\text { HM0134C } \\
\text { HM0135C }\end{array}$ & $\begin{array}{l}\mathbf{N} \\
\mathbf{N} \\
\mathbf{N} \\
\mathbf{N} \\
\mathbf{N} \\
\mathbf{N} \\
\mathbf{N} \\
\mathbf{N} \\
\mathbf{N}\end{array}$ & $\begin{array}{r}N \\
<20 \\
30 \\
N \\
N \\
30 \\
<20 \\
<20 \\
N \\
20\end{array}$ & $\begin{array}{r}700 \\
500 \\
3,000 \\
2,000 \\
150 \\
2,000 \\
200 \\
1,000 \\
>10,000 \\
300\end{array}$ & $\begin{array}{l}N \\
N \\
N \\
N \\
N \\
N \\
N \\
N \\
N \\
N\end{array}$ & $\begin{array}{l}N \\
N \\
N \\
N \\
N \\
N \\
N \\
N \\
N \\
N\end{array}$ & $\begin{array}{l}N \\
N \\
N \\
N \\
N \\
N \\
N \\
N \\
N \\
N\end{array}$ & $\begin{array}{l}\mathbf{N} \\
\mathbf{N} \\
\mathbf{N} \\
\mathbf{N} \\
\mathbf{N} \\
\mathbf{N} \\
\mathbf{N} \\
\mathbf{N} \\
\mathbf{N}\end{array}$ & $\begin{array}{r}700 \\
200 \\
150 \\
50 \\
20 \\
150 \\
700 \\
100 \\
300 \\
70\end{array}$ & $\begin{array}{l}N \\
N \\
N \\
N \\
N \\
N \\
N \\
N \\
N \\
N\end{array}$ \\
\hline $\begin{array}{l}\text { HM0136C } \\
\text { HM0137C } \\
\text { HM0138C } \\
\text { HMO139C } \\
\text { HM0140C } \\
\text { HM0141C } \\
\text { HMO142C } \\
\text { HMO143C } \\
\text { HMO144C } \\
\text { HMO145C }\end{array}$ & $\begin{array}{l}N \\
N \\
N \\
N \\
N \\
N \\
N \\
N \\
N \\
N\end{array}$ & $\begin{array}{r}20 \\
<20 \\
N \\
30 \\
<20 \\
30 \\
30 \\
<20 \\
50 \\
50\end{array}$ & $\begin{array}{r}300 \\
3,000 \\
500 \\
500 \\
300 \\
300 \\
700 \\
1,000 \\
300 \\
3,000\end{array}$ & $\begin{array}{l}<2 \\
N \\
N \\
N \\
N \\
N \\
N \\
<2 \\
N \\
N\end{array}$ & $\begin{array}{l}\mathbf{N} \\
\mathbf{N} \\
\mathbf{N} \\
\mathbf{N} \\
\mathbf{N} \\
\mathbf{N} \\
\mathbf{N} \\
\mathbf{N} \\
\mathbf{N}\end{array}$ & $\begin{array}{l}N \\
N \\
N \\
N \\
N \\
N \\
N \\
N \\
N \\
N\end{array}$ & $\begin{array}{l}N \\
N \\
N \\
N \\
N \\
N \\
N \\
N \\
N \\
N\end{array}$ & $\begin{array}{r}700 \\
150 \\
100 \\
70 \\
30 \\
150 \\
100 \\
100 \\
100 \\
300\end{array}$ & $\begin{array}{l}N \\
N \\
N \\
N \\
N \\
N \\
N \\
N \\
N \\
N\end{array}$ \\
\hline $\begin{array}{l}\text { HMO146C } \\
\text { HM0147C } \\
\text { HMO148C } \\
\text { HM0200C } \\
\text { HM0201C } \\
\text { HM0202C } \\
\text { HM0203C } \\
\text { HM0204C } \\
\text { HM0205C } \\
\text { HM0206C }\end{array}$ & $\begin{array}{l}\mathbf{N} \\
\mathbf{H} \\
\mathbf{H} \\
\mathbf{N} \\
\mathbf{N} \\
\mathbf{N} \\
\mathbf{N} \\
\mathbf{N} \\
\mathbf{N}\end{array}$ & $\begin{array}{r}<20 \\
50 \\
N \\
20 \\
30 \\
N \\
N \\
100 \\
20 \\
70\end{array}$ & $\begin{array}{r}7,000 \\
>10,000 \\
100 \\
200 \\
200 \\
200 \\
3,000 \\
150 \\
70 \\
150\end{array}$ & $\begin{array}{l}N \\
N \\
N \\
N \\
N \\
N \\
N \\
N \\
N \\
N\end{array}$ & $\begin{array}{r}M \\
N \\
N \\
N \\
N \\
N \\
N \\
N \\
1,500 \\
N\end{array}$ & $\begin{array}{l}N \\
N \\
N \\
N \\
N \\
N \\
N \\
N \\
N \\
N\end{array}$ & $\begin{array}{l}N \\
N \\
N \\
N \\
N \\
N \\
N \\
N \\
N \\
N\end{array}$ & $\begin{array}{r}50 \\
50 \\
150 \\
500 \\
500 \\
500 \\
70 \\
500 \\
300 \\
500\end{array}$ & $\begin{array}{l}N \\
N \\
N \\
N \\
N \\
N \\
N \\
N \\
N \\
N\end{array}$ \\
\hline $\begin{array}{l}\text { HMO207C } \\
\text { HM0208C } \\
\text { HM0209C } \\
\text { HM0210C } \\
\text { HM0211C } \\
\text { HM0211C } \\
\text { HM0213C } \\
\text { HM0214C } \\
\text { HM0215C } \\
\text { HMO216C }\end{array}$ & $\begin{array}{l}N \\
N \\
N \\
N \\
N \\
N \\
N \\
N \\
N \\
N\end{array}$ & $\begin{array}{r}N \\
N \\
N \\
N \\
N \\
200 \\
1,000 \\
N \\
N \\
20\end{array}$ & $\begin{array}{r}150 \\
100 \\
70 \\
100 \\
100 \\
150 \\
200 \\
300 \\
300 \\
300\end{array}$ & $\begin{array}{l}N \\
N \\
N \\
N \\
N \\
<2 \\
<2 \\
N \\
N \\
N\end{array}$ & $\begin{array}{r}N \\
N \\
N \\
N \\
N \\
500 \\
150 \\
N \\
N \\
N\end{array}$ & $\begin{array}{l}N \\
N \\
N \\
N \\
N \\
N \\
N \\
N \\
N \\
N\end{array}$ & $\begin{array}{l}N \\
N \\
N \\
N \\
N \\
N \\
N \\
N \\
N \\
N\end{array}$ & $\begin{array}{r}150 \\
100 \\
1,000 \\
300 \\
1,500 \\
1,500 \\
2,000 \\
200 \\
20 \\
500\end{array}$ & $\begin{array}{l}N \\
N \\
N \\
N \\
N \\
N \\
N \\
N \\
N \\
N\end{array}$ \\
\hline $\begin{array}{l}\text { HM0217C } \\
\text { HMO218C } \\
\text { HMO219C } \\
\text { HMO220C } \\
\text { HMO221C } \\
\text { HM0222C } \\
\text { HM0223C } \\
\text { HM0224C } \\
\text { HMO225C } \\
\text { HMO226C }\end{array}$ & $\begin{array}{l}N \\
N \\
N \\
N \\
N \\
N \\
N \\
N \\
N \\
N\end{array}$ & $\begin{array}{r}N \\
N \\
N \\
N \\
20 \\
<20 \\
<20 \\
<20 \\
20 \\
<20\end{array}$ & $\begin{array}{r}500 \\
2,000 \\
100 \\
200 \\
700 \\
700 \\
300 \\
500 \\
300 \\
300\end{array}$ & $\begin{array}{l}N \\
N \\
N \\
N \\
3 \\
N \\
N \\
N \\
N \\
N\end{array}$ & $\begin{array}{l}N \\
N \\
N \\
N \\
N \\
N \\
N \\
N \\
N \\
N\end{array}$ & $\begin{array}{l}N \\
N \\
N \\
N \\
N \\
N \\
N \\
N \\
N \\
N\end{array}$ & $\begin{array}{l}N \\
N \\
N \\
N \\
N \\
N \\
N \\
N \\
N \\
N\end{array}$ & $\begin{array}{r}70 \\
50 \\
30 \\
20 \\
150 \\
50 \\
70 \\
500 \\
100 \\
100\end{array}$ & $\begin{array}{l}N \\
N \\
N \\
N \\
N \\
N \\
N \\
N \\
N \\
N\end{array}$ \\
\hline $\begin{array}{l}\text { HMO2227C } \\
\text { HM0228C } \\
\text { HM0229C } \\
\text { HM0230C } \\
\text { HM0231C } \\
\text { HM0232C } \\
\text { HMO233C } \\
\text { HMO234C } \\
\text { HMO235C } \\
\text { HM0236C }\end{array}$ & $\begin{array}{l}N \\
N \\
N \\
N \\
N \\
N \\
N \\
N \\
N \\
N\end{array}$ & $\begin{array}{r}N \\
50 \\
<20 \\
50 \\
<20 \\
20 \\
30 \\
N \\
20 \\
N\end{array}$ & $\begin{array}{r}500 \\
200 \\
700 \\
200 \\
500 \\
1,000 \\
200 \\
500 \\
700 \\
1,000\end{array}$ & $\begin{array}{l}\mathbf{N} \\
\mathbf{N} \\
\mathbf{N} \\
\mathbf{N} \\
\mathbf{N} \\
\mathbf{N} \\
\mathbf{N} \\
\mathbf{N} \\
\mathbf{N} \\
\mathbf{N}\end{array}$ & $\begin{array}{l}\mathbf{N} \\
\mathbf{N} \\
\mathbf{N} \\
\mathbf{N} \\
\mathbf{N} \\
\mathbf{N} \\
\mathbf{N} \\
\mathbf{N} \\
\mathbf{N} \\
\mathbf{N}\end{array}$ & $\begin{array}{l}N \\
N \\
N \\
N \\
N \\
N \\
N \\
N \\
N \\
N\end{array}$ & $\begin{array}{l}N \\
N \\
N \\
N \\
N \\
N \\
N \\
N \\
N \\
N\end{array}$ & $\begin{array}{r}150 \\
50 \\
50 \\
30 \\
20 \\
100 \\
30 \\
20 \\
100 \\
100\end{array}$ & $\begin{array}{l}N \\
N \\
N \\
N \\
N \\
N \\
N \\
N \\
N \\
N\end{array}$ \\
\hline $\begin{array}{l}\text { HM0237C } \\
\text { HM0238C }\end{array}$ & $\begin{array}{l}N \\
N\end{array}$ & $\begin{array}{l}30 \\
20\end{array}$ & $\begin{array}{r}10,000 \\
200\end{array}$ & $\begin{array}{l}N \\
N\end{array}$ & $\begin{array}{l}\mathbf{N} \\
\mathbf{N}\end{array}$ & $\begin{array}{l}N \\
N\end{array}$ & $\begin{array}{l}N \\
N\end{array}$ & $\begin{array}{r}150 \\
70\end{array}$ & $\begin{array}{l}\mathbf{N} \\
\mathbf{N}\end{array}$ \\
\hline
\end{tabular}


Table 4. Analytical data for heavy-mineral-concentrate samples collected from the Horn Mountains area--Continued.

Sample Ga ppm-SaS Ge ppm-SaS La ppm-Sas Mn ppm-SaS Mo ppm-SaS Nb ppm-Sas Ni ppm-SaS Pb ppm-SaS Sb ppm-SaS Sc ppm-SaS

\begin{tabular}{|c|c|c|c|c|c|c|c|c|c|c|}
\hline $\begin{array}{l}\text { HM0115C } \\
\text { HM0116C } \\
\text { HM0117C } \\
\text { HM0118C } \\
\text { HM0119C } \\
\text { HM0120C } \\
\text { HM0121C } \\
\text { HM0122C } \\
\text { HM0123C } \\
\text { HM0124C }\end{array}$ & $\begin{array}{r}<10 \\
N \\
N \\
<10 \\
N \\
N \\
N \\
N \\
N \\
N\end{array}$ & $\begin{array}{l}N \\
N \\
N \\
N \\
N \\
N \\
N \\
N \\
N \\
N\end{array}$ & $\begin{array}{r}200 \\
100 \\
N \\
150 \\
150 \\
<100 \\
<100 \\
100 \\
<100 \\
500\end{array}$ & $\begin{array}{r}100 \\
50 \\
N \\
150 \\
70 \\
50 \\
30 \\
<20 \\
70 \\
150\end{array}$ & $\begin{array}{l}N \\
N \\
N \\
N \\
N \\
N \\
N \\
N \\
N \\
N\end{array}$ & $\begin{array}{r}N \\
N \\
<50 \\
N \\
50 \\
N \\
<50 \\
<50 \\
70 \\
N\end{array}$ & $\begin{array}{l}N \\
N \\
N \\
N \\
N \\
N \\
N \\
N \\
N \\
N\end{array}$ & $\begin{array}{r}50 \\
N \\
N \\
<20 \\
<20 \\
N \\
20 \\
N \\
20 \\
N\end{array}$ & $\begin{array}{r}N \\
N \\
N \\
<200 \\
N \\
N \\
N \\
N \\
N \\
N \\
N 200\end{array}$ & $\begin{array}{r}30 \\
<10 \\
50 \\
<10 \\
30 \\
10 \\
15 \\
10 \\
20 \\
70\end{array}$ \\
\hline $\begin{array}{l}\text { HM0125C } \\
\text { HM0126C } \\
\text { HM0128C } \\
\text { HM0129C } \\
\text { HM0130C } \\
\text { HM0131C } \\
\text { HM0132C } \\
\text { HM0133C } \\
\text { HM0134C } \\
\text { HM0135C }\end{array}$ & $\begin{array}{r}<10 \\
N \\
N \\
N \\
<10 \\
N \\
N \\
N \\
N \\
N\end{array}$ & $\begin{array}{l}N \\
N \\
N \\
N \\
N \\
N \\
N \\
N \\
N \\
N\end{array}$ & $\begin{array}{r}150 \\
100 \\
N \\
<100 \\
N \\
100 \\
100 \\
<100 \\
N \\
N\end{array}$ & $\begin{array}{r}70 \\
20 \\
N \\
20 \\
<20 \\
70 \\
50 \\
<20 \\
<20 \\
20\end{array}$ & $\begin{array}{l}N \\
N \\
N \\
N \\
N \\
N \\
N \\
N \\
N \\
N\end{array}$ & $\begin{array}{r}N \\
N \\
<50 \\
N \\
N \\
70 \\
50 \\
70 \\
50 \\
<50\end{array}$ & $\begin{array}{l}N \\
N \\
N \\
N \\
N \\
N \\
N \\
N \\
N \\
N\end{array}$ & $\begin{array}{r}<20 \\
<20 \\
<20 \\
N \\
N \\
N \\
N \\
N \\
N \\
N \\
N\end{array}$ & $\begin{array}{l}\mathbf{N} \\
N \\
N \\
N \\
N \\
N \\
N \\
N \\
N \\
N \\
N\end{array}$ & $\begin{array}{r}10 \\
N \\
<10 \\
<10 \\
10 \\
<10 \\
20 \\
N \\
10 \\
15\end{array}$ \\
\hline $\begin{array}{l}\text { HM0136C } \\
\text { HM0137C } \\
\text { HM0138C } \\
\text { HM0139C } \\
\text { HM0140C } \\
\text { HM0141C } \\
\text { HM0142C } \\
\text { HM0143C } \\
\text { HM0144C } \\
\text { HM0145C }\end{array}$ & $\begin{array}{r}N \\
N \\
N \\
N \\
<10 \\
N \\
N \\
N \\
N \\
N \\
N\end{array}$ & $\begin{array}{l}N \\
N \\
N \\
N \\
N \\
N \\
N \\
N \\
N \\
N\end{array}$ & $\begin{array}{r}<100 \\
N \\
100 \\
<100 \\
100 \\
150 \\
100 \\
300 \\
<100 \\
150\end{array}$ & $\begin{array}{r}20 \\
30 \\
50 \\
20 \\
30 \\
<20 \\
20 \\
N \\
30 \\
20\end{array}$ & $\begin{array}{l}N \\
N \\
N \\
N \\
N \\
N \\
N \\
N \\
N \\
N\end{array}$ & $\begin{array}{r}50 \\
50 \\
<50 \\
N \\
N \\
100 \\
50 \\
<50 \\
N \\
<50\end{array}$ & $\begin{array}{l}N \\
N \\
N \\
N \\
N \\
N \\
N \\
N \\
N \\
N\end{array}$ & $\begin{array}{r}<20 \\
N \\
<20 \\
N \\
N \\
<20 \\
N \\
<20 \\
<20 \\
N\end{array}$ & $\begin{array}{l}N \\
N \\
N \\
N \\
N \\
N \\
N \\
N \\
N \\
N\end{array}$ & $\begin{array}{r}30 \\
<10 \\
10 \\
10 \\
N \\
20 \\
<10 \\
<10 \\
<10 \\
<10\end{array}$ \\
\hline $\begin{array}{l}\text { HM0146C } \\
\text { HM0147C } \\
\text { HM0148C } \\
\text { HM0200C } \\
\text { HM0201C } \\
\text { HM0202C } \\
\text { HM0203C } \\
\text { HM0204C } \\
\text { HM0205C } \\
\text { HM0206C }\end{array}$ & $\begin{array}{r}N \\
N \\
N \\
N \\
N \\
N \\
N \\
<10 \\
N \\
N \\
N\end{array}$ & $\begin{array}{l}N \\
N \\
N \\
N \\
N \\
N \\
N \\
N \\
N \\
N\end{array}$ & $\begin{array}{r}N \\
100 \\
N \\
150 \\
<100 \\
N \\
N \\
150 \\
100 \\
150\end{array}$ & $\begin{array}{r}<20 \\
<20 \\
<20 \\
70 \\
70 \\
20 \\
<20 \\
50 \\
150 \\
70\end{array}$ & $\begin{array}{l}N \\
N \\
N \\
N \\
N \\
N \\
N \\
N \\
N \\
N\end{array}$ & $\begin{array}{r}N \\
<50 \\
<50 \\
N \\
N \\
<50 \\
N \\
<50 \\
50 \\
<50\end{array}$ & $\begin{array}{l}N \\
N \\
N \\
N \\
N \\
N \\
N \\
N \\
N\end{array}$ & $\begin{array}{r}N \\
N \\
N \\
70 \\
<20 \\
200 \\
N \\
20 \\
500 \\
N\end{array}$ & $\begin{array}{r}N \\
N \\
N \\
200 \\
N \\
200 \\
N \\
N \\
N \\
N\end{array}$ & $\begin{array}{r}<10 \\
<10 \\
<10 \\
30 \\
30 \\
10 \\
10 \\
15 \\
<10 \\
<10\end{array}$ \\
\hline $\begin{array}{l}\text { HM0207C } \\
\text { HM0208C } \\
\text { HM0209C } \\
\text { HM0210C } \\
\text { HM0211C } \\
\text { HM0212C } \\
\text { HM0213C } \\
\text { HM0214C } \\
\text { HM0215C } \\
\text { HM0216C }\end{array}$ & $\begin{array}{r}N \\
N \\
N \\
N \\
N \\
N \\
N \\
N \\
N \\
<10\end{array}$ & $\begin{array}{l}N \\
N \\
N \\
N \\
N \\
N \\
N \\
N \\
N \\
N\end{array}$ & $\begin{array}{r}100 \\
100 \\
N \\
200 \\
<100 \\
N \\
<100 \\
<100 \\
<100 \\
150\end{array}$ & $\begin{array}{r}50 \\
50 \\
<20 \\
200 \\
50 \\
50 \\
50 \\
50 \\
30 \\
70\end{array}$ & $\begin{array}{l}N \\
N \\
N \\
N \\
N \\
N \\
N \\
N \\
N \\
N\end{array}$ & $\begin{array}{r}<50 \\
N \\
50 \\
N \\
<50 \\
<50 \\
50 \\
N \\
N \\
<50\end{array}$ & $\begin{array}{l}N \\
N \\
N \\
N \\
N \\
N \\
N \\
N \\
N \\
N\end{array}$ & $\begin{array}{r}N \\
N \\
<20 \\
N \\
20 \\
500 \\
N \\
<20 \\
N \\
N\end{array}$ & $\begin{array}{r}N \\
N \\
N \\
N \\
N \\
200 \\
N \\
N \\
N \\
N \\
N\end{array}$ & $\begin{array}{r}10 \\
<10 \\
70 \\
15 \\
20 \\
10 \\
70 \\
10 \\
10 \\
10\end{array}$ \\
\hline $\begin{array}{l}\text { HM0217C } \\
\text { HM0218C } \\
\text { HM0219C } \\
\text { HM0220C } \\
\text { HM0221C } \\
\text { HM0222C } \\
\text { HM0223C } \\
\text { HM0224C } \\
\text { HM0225C } \\
\text { HM0226C }\end{array}$ & $\begin{array}{r}N \\
N \\
N \\
N \\
10 \\
<10 \\
N \\
N \\
N \\
N\end{array}$ & $\begin{array}{l}N \\
N \\
N \\
N \\
N \\
N \\
N \\
N \\
N \\
N\end{array}$ & $\begin{array}{r}<100 \\
N \\
N \\
N \\
<100 \\
N \\
150 \\
N \\
<100 \\
N\end{array}$ & $\begin{array}{r}50 \\
<20 \\
<20 \\
20 \\
70 \\
20 \\
30 \\
N \\
<20 \\
<20\end{array}$ & $\begin{array}{l}N \\
N \\
N \\
N \\
N \\
N \\
N \\
N \\
N \\
N\end{array}$ & $\begin{array}{r}N \\
<50 \\
<50 \\
N \\
<50 \\
N \\
<50 \\
70 \\
<50 \\
70\end{array}$ & $\begin{array}{l}N \\
N \\
N \\
N \\
N \\
N \\
N \\
N \\
N \\
N\end{array}$ & $\begin{array}{r}N \\
N \\
N \\
N \\
N \\
N \\
<20 \\
<20 \\
N \\
N\end{array}$ & $\begin{array}{l}N \\
N \\
N \\
N \\
N \\
N \\
N \\
N \\
N \\
N\end{array}$ & $\begin{array}{r}10 \\
<10 \\
10 \\
30 \\
20 \\
10 \\
N \\
15 \\
30 \\
<10\end{array}$ \\
\hline $\begin{array}{l}\text { HMO227C } \\
\text { HM0228C } \\
\text { HMO229C } \\
\text { HM0230C } \\
\text { HM0231C } \\
\text { HMO232C } \\
\text { HM0233C } \\
\text { HM0234C } \\
\text { HM0235C } \\
\text { HMO236C }\end{array}$ & $\begin{array}{l}N \\
N \\
N \\
N \\
N \\
N \\
N \\
N \\
N \\
N\end{array}$ & $\begin{array}{l}N \\
N \\
N \\
N \\
N \\
N \\
N \\
N \\
N \\
N\end{array}$ & $\begin{array}{r}N \\
150 \\
<100 \\
100 \\
<100 \\
N \\
<100 \\
N \\
100 \\
N\end{array}$ & $\begin{array}{r}<20 \\
30 \\
30 \\
70 \\
20 \\
<20 \\
20 \\
<20 \\
<20 \\
<20\end{array}$ & $\begin{array}{l}N \\
N \\
N \\
N \\
N \\
N \\
N \\
N \\
N \\
N\end{array}$ & $\begin{array}{r}N \\
<50 \\
<50 \\
<50 \\
<50 \\
50 \\
<50 \\
N \\
N \\
<50\end{array}$ & $\begin{array}{l}N \\
N \\
N \\
N \\
N \\
N \\
N \\
N \\
N \\
N\end{array}$ & $\begin{array}{l}N \\
N \\
N \\
N \\
N \\
N \\
N \\
N \\
N \\
N\end{array}$ & $\begin{array}{l}N \\
N \\
N \\
N \\
N \\
N \\
N \\
N \\
N \\
N\end{array}$ & $\begin{array}{r}<10 \\
15 \\
<10 \\
10 \\
15 \\
<10 \\
15 \\
<10 \\
20 \\
10\end{array}$ \\
\hline $\begin{array}{l}\text { HM0237C } \\
\text { HM0238C }\end{array}$ & $\begin{array}{l}N \\
N\end{array}$ & $\begin{array}{l}N \\
N\end{array}$ & $\begin{array}{r}150 \\
N\end{array}$ & $\begin{array}{l}20 \\
30\end{array}$ & $\begin{array}{l}N \\
N\end{array}$ & $\begin{array}{l}<50 \\
<50\end{array}$ & N & $\begin{array}{r}N \\
<20\end{array}$ & $\begin{array}{l}N \\
N\end{array}$ & $\begin{array}{r}20 \\
N\end{array}$ \\
\hline
\end{tabular}


Table 4. Analytical data for heavy-mineral-concentrate samples collected from the Horn Mountains area--Continued.

Sample Sn ppm-SQS Sr ppm-SQS Th ppm-SQS V ppm-SQS W ppm-SQS Y ppm-SQS Zn ppm-SQS Zr ppm-SQS Pd ppm-SQS Pt ppm-SQS

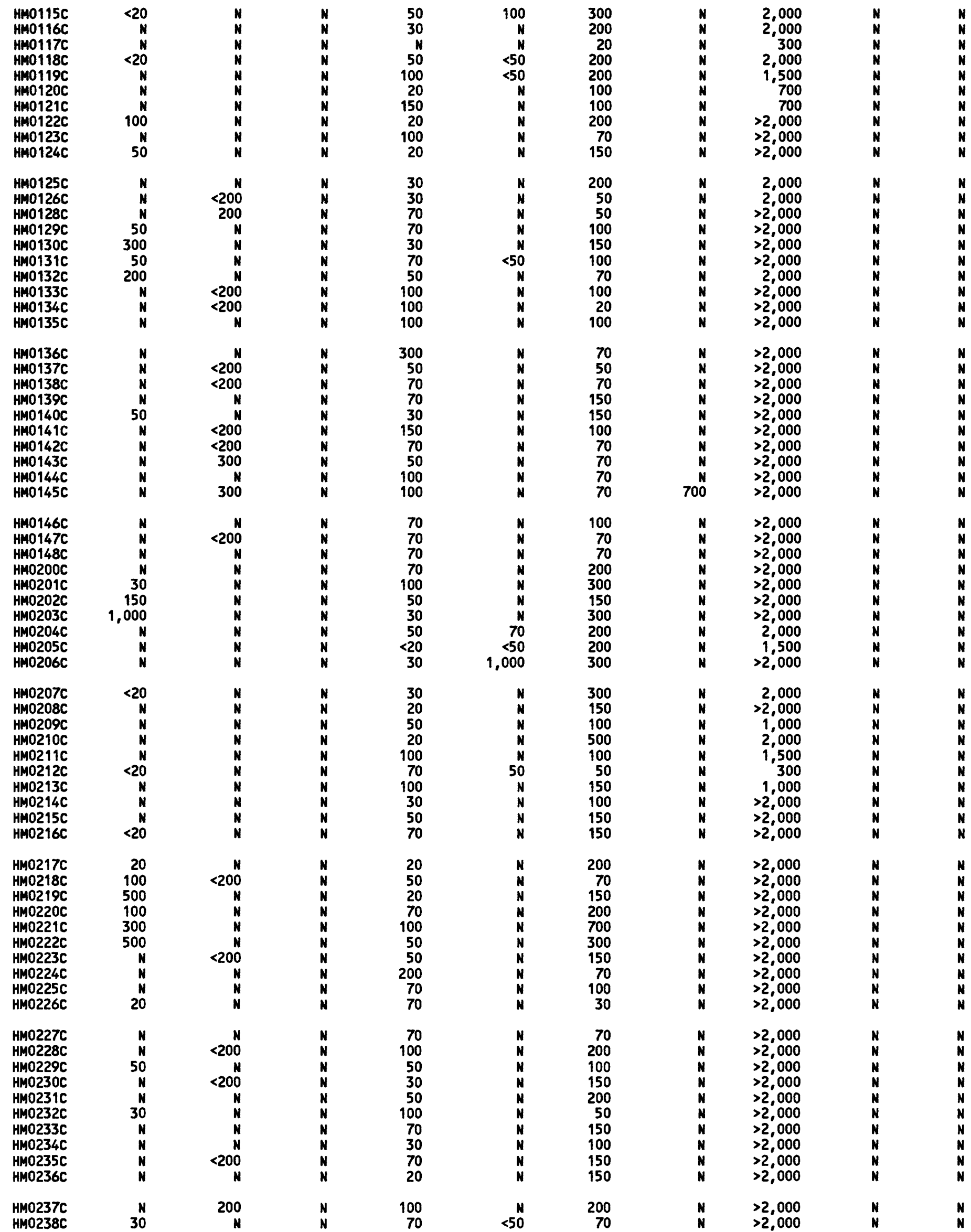


momomuminon unNogmmmona

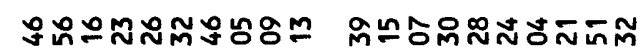

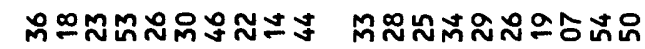

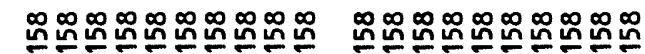

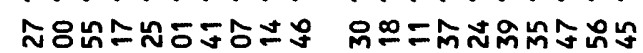

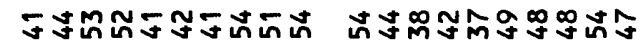

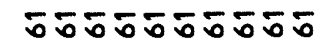

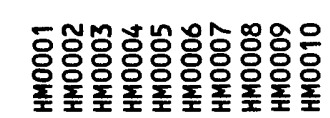
5555555.55

$00000000-0$ $0-00-00000$ $000-00000 N$ odoonomon $0--0-0000$ $-000000000$ $000000-000$ 0000000000 $0-00000000$ 0000000000 $0-r-0-000$ 0000000000 0000000000 $00---0-n$ $--n-0-00-$ $00000000-0$ 0000000000 nMUUSMnNT - MUMmNNTM $0-0-000000$ MUNMNMUNNM mmovomenLmo

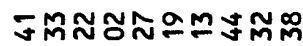

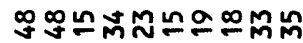

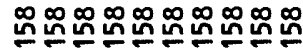

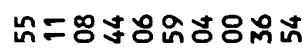

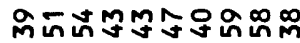
$\overline{0} 5 \overline{5} 5 \overline{5} 5 \overline{5} 5 \overline{5}$

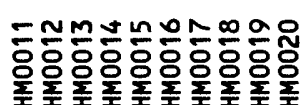

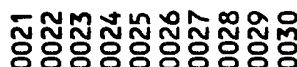

$00-0000-00$ $00--0-0-0$

0000000000 0000000000 0000000000 $-m 0000-000$ -ON-T- ONT $000-00000-$ 0000000000 $-00000-000$ $--000000$ $00000-0000$ 0000000000 $0-r-00-0-N$ 0000000000 0000000000 - N-N-N-N-n-mo-n $00-0000000$ 0000000000 $0-0-00-00-$ mmmusougun NommMMNMMM - NOTO-0000 0000000000 N-M-NNNNNN

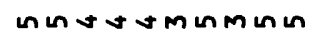

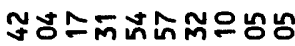

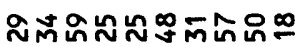

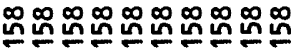

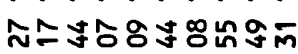

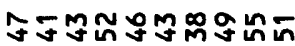
5555555555

$-m-N m N N-N-$ nonmovinmoun

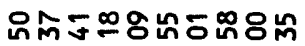
느ํํํำำํํำ

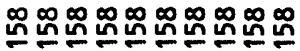

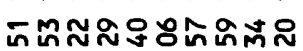

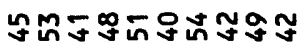
ธธธธธธธธธธธธธ

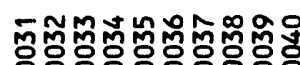

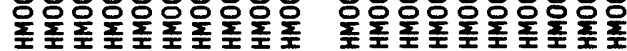

0000000000 $000-000000$ 0000000000 $-000000000$ $00000000-0$ 0000000000 $-000000000$ 0000000000 0000000000 0000000000 -OT-ONMOMN $000000-00-$ 0000000000 n- - N N NNN 


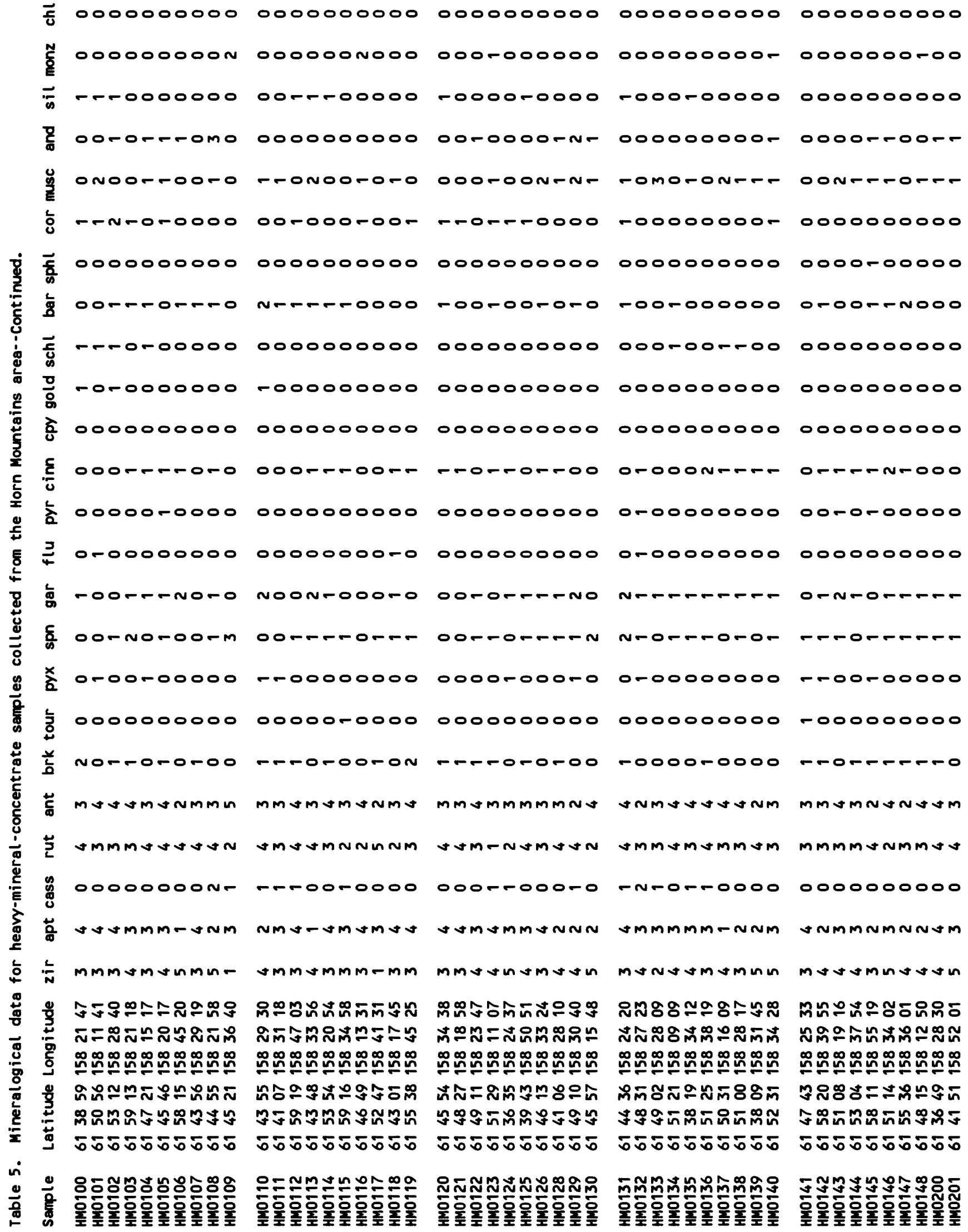


올

$\frac{\pi}{6}$$$
\text { 高 }
$$$$
\text { 是 }
$$$$
\text { 농 }
$$$$
\text { - }
$$

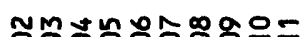

0000000000

0000000000

0000000

$00000-0000$

0000000000

0000000

0-0r-0000-

$00000000-0$

$00000-0$

$000-000-00$

$m-000-0000$

$000-000$

- - 0-00-r-o

montumn

$n-r-m$

0-m000000-

0000000000

0000000000

0000000

$1000+0000$

$-000000-00$

-000000000

0000000000

0000000000

0000000

0000000000

000000000

0000000

$--m-r-000$

$0000000000000-0-000000000-0$

-oooonor-r

oornnth

$-00000000000000000000000000$

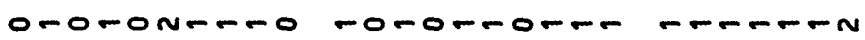

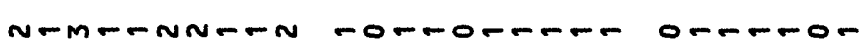

-00000000 00000000000000000

$-00000000--000000000000000$

n-m-O-OO

-00--000-0

o0m-wo

$\checkmark m m m m m m+m$

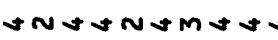

$m m m \cup m \leq m$

nMvmunamam

nmavammmu

amananat

No000-0-0.

$00000-0-00$

ooro-ro

vonmmommnm

NNNNNNMMNM

-mmmmmn

movommonmm

mmotatamatand

montmon

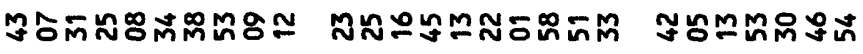

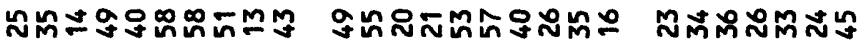

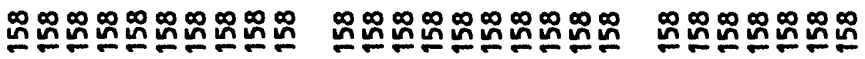

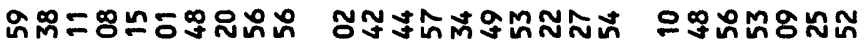

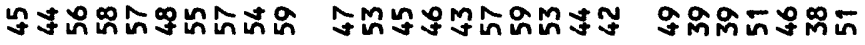
$5 \overline{0} 5 \overline{0} 5 \overline{0} 5 \overline{0} 5 \overline{0} 55555550555555$

Mレํำ윰

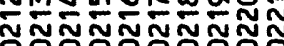

NM⿻上丨

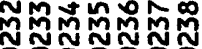

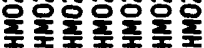

